\title{
Trefftz-based methods for time-harmonic acoustics
}

\author{
B. Pluymers, B. van Hal, D. Vandepitte, W. Desmet \\ Katholieke Universiteit Leuven \\ Department of Mechanical Engineering \\ Celestijnenlaan 300 B \\ B-3001 Heverlee, Belgium \\ bert.pluymers@mech.kuleuven.be
}

\begin{abstract}
Summary
Over the last decade, Computer Aided Engineering (CAE) tools have become essential in the assessment and optimization of the acoustic characteristics of products and processes. The possibility of evaluating these characteristics on virtual prototypes at almost any stage of the design process reduces the need for very expensive and time consuming physical prototype testing. However, despite their steady improvements and extensions, CAE techniques are still primarily used by analysis specialists. In order to turn them into easyto-use, versatile tools that are also easily accessible for designers, several bottlenecks have to be resolved. The latter include, amongst others, the lack of efficient numerical techniques for solving system-level functional performance models in a wide frequency range. This paper reviews the CAE modelling techniques which can be used for the analysis of time-harmonic acoustic problems and focusses on techniques which have the Trefftz approach as baseline methodology. The basic properties of the different methods are highlighted and their strengths and limitations are discussed. Furthermore, an overview is given of the state-of-the-art of the extensions and the enhancements which have been recently investigated to enlarge the application range of the different techniques. Specific attention is paid to one very promising Trefftz-based technique, which is the so-called wave based method. This method has all the necessary attributes for putting a next step in the evolution towards truly virtual product design.
\end{abstract}

\section{INTRODUCTION}

This paper reviews the research efforts spent on the development of numerical methods for the analysis of time-harmonic acoustics, with a specific focus on Trefftz-based methods. Acoustics refers to the engineering field, which considers small pressure perturbations with respect to a constant ambient pressure level. The small pressure perturbations, or acoustic pressures, exhibit both a spatial and a temporal distribution. In many cases, the acoustic processes of interest are stationary, i.e. they show a time-harmonic behaviour, for which the description in the frequency domain, or in the wave number domain, is often more suitable than in the time domain. At discrete frequencies, the linear (or linearized) Helmholtz equation governs the spatial distribution of the acoustic pressures $[1,2]$.

Many deterministic methods exist for solving linear Helmholtz equations. Among those methods, the finite element method (FEM) $[3,4]$ and the boundary element method (BEM) $[5,6,7]$ are probably the most well known. These methods perform satisfactory for the low-frequency range, i.e. for those frequencies for which the corresponding characteristic wavelengths are of the same order of magnitude as the dimensions of the bounded domain. However, one of the major computational challenges nowadays is the treatise of high-frequency or short-wave phenomena [8]. This topic is therefore investigated extensively, which is apparent through the large number of dedicated conference sessions $[9,10,11,12,13]$ and review papers $[14,15,16]$.

A large diversity of computational methods for high-frequency problems in acoustics coexist. Their number is too large to thoroughly discuss them all. Nevertheless, in this paper an attempt is made to classify the methods roughly in some general categories. One of these categories will be detailed further, namely the class of the Trefftz-based methods. 
The basic principles of these methods date back to 1926 when Trefftz [17] presented an alternative for the Rayleigh-Ritz approach, on which for example the FEM is based. The Trefftz-based approach has been unnoticed for a long time. Only in the 1970s, the quest for more accurate FEMs drives the growing interest for the Trefftz-based method. In the years that follow, the value of the Trefftz-based methods has been proven for applications in elasticity and heat transfer, for plate bending and shell problems and for the Poisson problem. For more details, the reader is referred to the review papers [18] and [19], both published in the mid-1990s. Since the mid-1990s, the application of the Trefftz-based methods has been extended for time-harmonic problems in acoustics, structural mechanics and electro-magnetics in order to meet the challenges of the high-frequency phenomena.

The objective of this paper is to give an overview of the state-of-the-art of the element based and the Trefftz-based methods for the analysis of time-harmonic acoustic problems. In addition, special attention is paid to a particular Trefftz-based method, namely the so-called wave based method, on which the authors have provided a substantial scientific contribution $[20,21,22,23,24]$.

This paper is outlined as follows. The mathematical model for time-harmonic acoustic problems is presented first. In order to illustrate the computational challenges with respect to high-frequency phenomena, the basic features of the FEM are outlined including a discussion on the numerical accuracy. This discussion clears the way for categorizing the enhancements of the classical FEM, such as the stabilized approaches, the generalized methods, the multi-scale approaches, etcetera. In a comparable layout, a next section discusses the BEM and its extensions and enhancements. Then, a detailed survey of the Trefftz-based methods is provided. In particular the capabilities of the the WBM for simulating high-frequency acoustic problems are discussed in detail and demonstrated for both interior and exterior acoustic problems.

\section{MATHEMATICAL MODEL}

\subsection{Boundary value problem}

Figure 1 shows a bounded domain $\Omega_{i}$ with the following acoustic properties: the ambient density $\rho$ and the speed of sound $c$. A point source at position $\mathbf{r}_{q}$, characterized by a sourcestrength amplitude $q$, excites the acoustic domain. The excitation has a time-harmonic behaviour $\mathrm{e}^{j \omega t}$ with a radial frequency $\omega$. The response consists of the acoustic pressure $p$ and the particle velocity $\mathbf{v}$, which also show a time-harmonic behaviour with the frequency $\omega$. The following set of coupled partial differential equations governs the time-harmonic

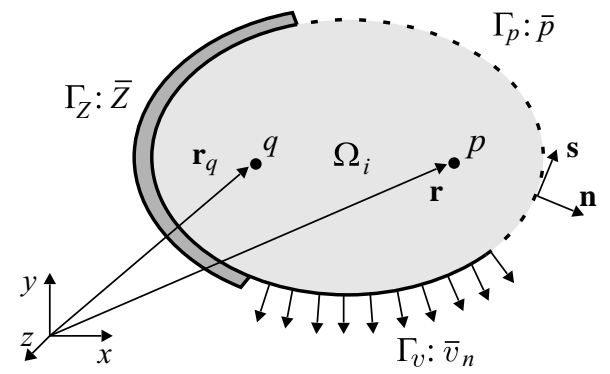

Figure 1: Interior acoustic problem

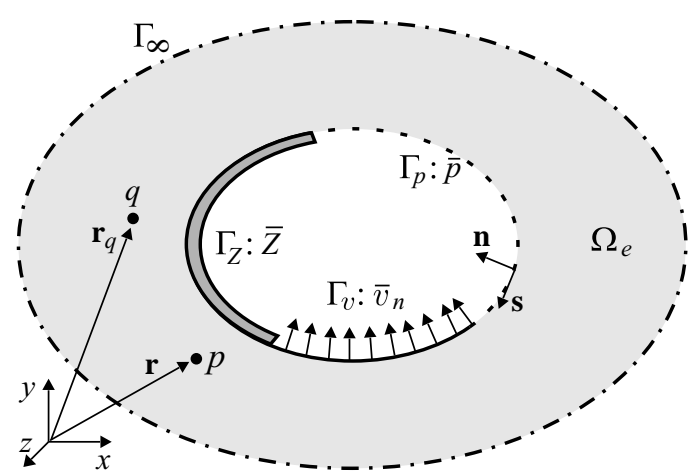

Figure 2: Exterior acoustic problem 
acoustic pressure $p$ and the particle velocity $\mathbf{v}$ at position $\mathbf{r}[1,2]$

$$
\begin{array}{cll}
\text { conservation of mass : } & \nabla^{T} \mathbf{v}(\mathbf{r})+\frac{j k}{\rho c} p(\mathbf{r})=q \delta\left(\mathbf{r}, \mathbf{r}_{q}\right), & \forall \mathbf{r} \in \Omega_{i}, \\
\text { equation of motion: } & \nabla p(\mathbf{r})+j \rho \omega \mathbf{v}(\mathbf{r})=0, & \forall \mathbf{r} \in \Omega_{i} .
\end{array}
$$

In these equations, $\boldsymbol{\nabla}=\left[\begin{array}{lll}\partial / \partial x & \partial / \partial y & \partial / \partial z\end{array}\right]^{T}$ represents the gradient operator, where the superscript ${ }^{T}$ denotes the transpose, $k=\omega / c$ represents the wave number, $j=\sqrt{-1}$ the unit imaginary number and $\delta$ a Dirac delta function. The conservation of mass and the equation of motion are usually combined resulting in the linear Helmholtz equation

$$
\left(\Delta+k^{2}\right) p(\mathbf{r})=-j \rho \omega q \delta\left(\mathbf{r}, \mathbf{r}_{q}\right), \quad \forall \mathbf{r} \in \Omega_{i},
$$

where $\Delta=\partial^{2} / \partial x^{2}+\partial^{2} / \partial y^{2}+\partial^{2} / \partial z^{2}$ is the Laplace operator. Since the Helmholtz equation is a second-order partial differential equation, one boundary condition is required at each boundary point in order for the acoustic problem to be well-posed. The following timeharmonic, acoustic boundary conditions may be imposed at the problem boundary $\partial \Omega_{i}=$ $\Gamma_{p} \cup \Gamma_{v} \cup \Gamma_{Z}$

$$
\begin{aligned}
p(\mathbf{r}) & =\bar{p}(\mathbf{r}), & & \forall \mathbf{r} \in \Gamma_{p}, \\
\mathbf{n}^{T} \mathbf{v}(\mathbf{r}) & =\bar{v}_{n}(\mathbf{r}), & & \forall \mathbf{r} \in \Gamma_{v}, \\
\mathbf{n}^{T} \mathbf{v}(\mathbf{r}) & =p(\mathbf{r}) / \bar{Z}(\mathbf{r}), & & \forall \mathbf{r} \in \Gamma_{Z} .
\end{aligned}
$$

In these relations, $\mathbf{n}$ is the outward normal vector at the problem boundary (see figure 1 ) and $\bar{p}, \bar{v}_{n}$ and $\bar{Z}$ are prescribed distributions for the pressure, the normal velocity and the normal impedance along the corresponding parts of the problem boundary.

The above mathematical model is a boundary value problem for interior acoustics. The modelling of exterior problems, e.g. sound radiation problems, follows the same principles. Figure 2 shows an unbounded domain $\Omega_{e}$ with the acoustic properties $\rho$ and $c$. Again, a point source $q$ excites the acoustic domain and the Helmholtz equation (2) governs the time-harmonic response. However, now the problem boundary $\partial \Omega_{e}$ is composed of (i) a finite part $\Gamma_{p} \cup \Gamma_{v} \cup \Gamma_{Z}$, where the boundary conditions of equation (3) are imposed, and of (ii) an unbounded part $\Gamma_{\infty}$, where the Sommerfeld radiation condition [25] is imposed to model the far field correctly

$$
\lim _{r \rightarrow \infty} r\left(\frac{\partial p(\mathbf{r})}{\partial r}+j k p(\mathbf{r})\right)=0, \quad \text { with } r=\|\mathbf{r}\| .
$$

A two-dimensional (2D) acoustic problem can be derived from the above full 3D representations if the following conditions are satisfied.

1. The dimension of the acoustic domain $\Omega_{\{i / e\}}$ is infinite in the $z$-direction.

2. The boundary conditions do not vary in the $z$-direction.

3. A line source at position $\left(x_{q}, y_{q}\right)$, characterized by a source-strength amplitude $q$ excites the acoustic system.

4. In case of an exterior acoustic problem, the $2 \mathrm{D}$ expression of the Sommerfeld radiation condition is selected

$$
\lim _{r \rightarrow \infty} \sqrt{r}\left(\frac{\partial p(\mathbf{r})}{\partial r}+j k p(\mathbf{r})\right)=0 .
$$


B. Pluymers, B. van Hal, D. Vandepitte and W. Desmet

The $3 \mathrm{D}$ problem reduces to a $2 \mathrm{D}$ problem by omitting the $z$-dependency in the involved linear operators.

The discussed boundary value problems can be solved numerically using the FEM, the BEM or the Trefftz-based methods. The FEM and the Trefftz-based methods make direct use of the governing differential equations. The BEM, on the other hand, requires that the boundary value problem is described by boundary integral formulations. These methods are reviewed next.

\subsection{Boundary integral formulation}

The following inhomogeneous integral equation of the second kind is applicable to describe both interior and exterior acoustic problems

$$
C(\mathbf{r}) p(\mathbf{r})=g\left(\mathbf{r}, \mathbf{r}_{q}, k\right)+\int_{\Gamma_{f}}\left(p\left(\mathbf{r}_{b}\right) \frac{\partial g\left(\mathbf{r}, \mathbf{r}_{b}, k\right)}{\partial n}+j \rho \omega v_{n}\left(\mathbf{r}_{b}\right) g\left(\mathbf{r}, \mathbf{r}_{b}, k\right)\right) \mathrm{d} \Gamma\left(\mathbf{r}_{b}\right) .
$$

This boundary integral equation relates the pressure $p$ at position $\mathbf{r}$ to the pressure distribution $p$ and normal velocity distribution $v_{n}$ along the finite part of the domain boundary $\Gamma_{f}=\Gamma_{p} \cup \Gamma_{v} \cup \Gamma_{Z}$. The equation is referred to as the direct boundary integral since the involved boundary values are the acoustic quantities of interest. In equation (6), the vector $\mathbf{r}_{b}$ indicates the position of the boundary points. The $\partial / \partial n=\mathbf{n}^{T} \boldsymbol{\nabla}$ operator represents the gradient operator in the outward normal direction $\mathbf{n}$. The free-field Green's function $g\left(\mathbf{r}, \mathbf{r}_{q}, k\right)$ takes the effect of the acoustic line source (2D) or of the acoustic point source (3D) at position $\mathbf{r}_{q}$ into account. The free-field Green's function is a fundamental solution of the inhomogeneous Helmholtz equation (2) given by

$$
g\left(\mathbf{r}, \mathbf{r}_{q}, k\right)= \begin{cases}\frac{-j}{4} H_{o}^{(2)}\left(k\left\|\mathbf{r}-\mathbf{r}_{q}\right\|\right), & (2 D) \\ \frac{\mathrm{e}^{-j k\left\|\mathbf{r}-\mathbf{r}_{q}\right\|}}{4 \pi\left\|\mathbf{r}-\mathbf{r}_{q}\right\|}, & (3 D)\end{cases}
$$

where $H_{o}^{(2)}$ represents the zero-order Hankel function of the second kind. The free-field Green's function is also used as kernel function $g\left(\mathbf{r}, \mathbf{r}_{b}, k\right)$ in the boundary integral equation (6). The position dependent coefficient $C(\mathbf{r})$ in this equation is given by

$$
C(\mathbf{r})= \begin{cases}1, & \forall \mathbf{r} \in\left\{\Omega \backslash \Gamma_{f}\right\} \\ \frac{1}{2}, & \forall \mathbf{r} \in \Gamma_{f} \\ 0, & \forall \mathbf{r} \notin \Omega\end{cases}
$$

Note that the value $\frac{1}{2}$ holds for the boundary points $\mathbf{r}_{b}$ where the normal vector $\mathbf{n}$ is continuous. Otherwise the coefficient $C\left(\mathbf{r}_{b}\right)$ takes a value between 0 and 1 depending on the discontinuity or the jump of the normal vector $\llbracket \mathbf{n}\left(\mathbf{r}_{b}\right) \rrbracket$. The derivation of the direct boundary integral equation is too lengthy to present here. The interested reader is referred to $[5,6,7,26]$ for the details.

A second type of boundary integral formulation is based on the indirect boundary integral equation given by

$$
p(\mathbf{r})=g\left(\mathbf{r}, \mathbf{r}_{q}, k\right)+\int_{\Gamma_{f}}\left(-\sigma\left(\mathbf{r}_{b}\right) g\left(\mathbf{r}, \mathbf{r}_{b}, k\right)+\mu\left(\mathbf{r}_{b}\right) \frac{\partial g\left(\mathbf{r}, \mathbf{r}_{b}, k\right)}{\partial n}\right) \mathrm{d} \Gamma\left(\mathbf{r}_{b}\right) .
$$


The term 'indirect' refers to the boundary values $\sigma\left(\mathbf{r}_{b}\right)$ and $\mu\left(\mathbf{r}_{b}\right)$, which are not the acoustic variables of interest, i.e. $p$ and $v_{n}$. However, the boundary values represent the densities of continuously distributed sources along the finite part of the boundary $\Gamma_{f}$.

Recall that $g\left(\mathbf{r}, \mathbf{r}_{b}, k\right)$ is the free-field pressure response for a point source at position $\mathbf{r}_{b}$. In the indirect boundary integral equation (9), an infinite number of point sources are distributed along the boundary $\Gamma\left(\mathbf{r}_{b}\right)$ with the density $\sigma\left(\mathbf{r}_{b}\right)$. The integration along the boundary represents their combined effect on the pressure field and is usually referred to as the single layer potential. Similarly, it is not difficult to see that $\partial g\left(\mathbf{r}, \mathbf{r}_{b}, k\right) / \partial n$ represents the free-field pressure response for a dipole source at position $\mathbf{r}_{b}$. The integration of an infinite number of dipole sources with the density $\mu\left(\mathbf{r}_{b}\right)$ results in the double layer potential.

The indirect boundary integral formulation allows one also to consider combined interior/exterior acoustic problems and acoustic problems with open boundaries. For these types of problems, the single and double layer potential densities $\sigma\left(\mathbf{r}_{b}\right)$ and $\mu\left(\mathbf{r}_{b}\right)$ are related as follows to the acoustic variables

$$
\begin{aligned}
\sigma\left(\mathbf{r}_{b}\right) & =\frac{j}{\rho \omega}\left(v_{n}\left(\mathbf{r}_{b}^{+}\right)-v_{n}\left(\mathbf{r}_{b}^{-}\right)\right), \\
\mu\left(\mathbf{r}_{b}\right) & =p\left(\mathbf{r}_{b}^{+}\right)-p\left(\mathbf{r}_{b}^{-}\right)
\end{aligned}
$$

The $\mathbf{r}_{b}^{+}$vector indicates the boundary position $\mathbf{r}_{b}$ at the positive side of the boundary, i.e., the side in which the normal vector $\mathbf{n}$ is directed. The $\mathbf{r}_{b}^{-}$vector indicates the opposite, negative side.

The solutions of the boundary integral equations in (6) and (9) satisfy the Sommerfeld radiation condition a priori (although this is irrelevant for the bounded problems). These solutions are obtained in two steps.

1. The boundary integrals are evaluated along the boundary $\Gamma_{f}$. This requires the substitution of the boundary conditions resulting in a boundary integral for the unknown boundary values. For example, in case of the direct formulation with a prescribed normal velocity distribution $\bar{v}_{n}\left(\mathbf{r}_{b}\right)$, this integral becomes

$$
\frac{1}{2} p(\mathbf{r})=g\left(\mathbf{r}, \mathbf{r}_{q}, k\right)+\int_{\Gamma_{f}}\left(p\left(\mathbf{r}_{b}\right) \frac{\partial g\left(\mathbf{r}, \mathbf{r}_{b}, k\right)}{\partial n}+j \rho \omega \bar{v}_{n}\left(\mathbf{r}_{b}\right) g\left(\mathbf{r}, \mathbf{r}_{b}, k\right)\right) \mathrm{d} \Gamma\left(\mathbf{r}_{b}\right), \forall \mathbf{r} \in \Gamma_{f} .
$$

2. All boundary values are known from the previous step. The pressure at an arbitrary position $\mathbf{r}$ follows from the evaluation of the considered boundary integral equation.

\section{FINITE ELEMENT METHOD}

\subsection{Basic formulations}

The finite element method (FEM) has proven to be one of the most suitable computational methods for solving engineering problems, which consist of finding the spatial and possibly temporal distribution of physical quantities in a continuous medium [3]. Its application for acoustic problems is well developed [4]. This section outlines the basic formulation of the method rather detailed. The reason for this is twofold. The numerical difficulties, which arise in high-frequency acoustics, can be clearly illustrated and many methods nowadays consist of enhancing the classical FEM.

Consider the interior acoustic problem, which is fully described by equations (1)-(3). The exact solution of this problem is approximated by employing the following finite element (FE) strategy: 
1. The bounded domain $\Omega_{i}$ is subdivided in subdomains $\left(\Omega_{i}=\cup_{e=1}^{n_{e}} \Omega^{e}\right.$ with $\Omega^{e} \cap \Omega^{g}=0$, $\forall e \neq g$ ), i.e. the finite elements $\Omega^{e}$ as shown in figure 3. The boundary of each element $\partial \Omega^{e}=\Gamma_{p}^{e} \cup \Gamma_{v}^{e} \cup \Gamma_{Z}^{e} \cup \Gamma_{i}^{e}$ is composed of the intersections with the problem boundary, e.g. $\Gamma_{p}^{e}=\partial \Omega^{e} \cap \Gamma_{p}$, and the common interface $\Gamma_{i}^{e}$ between two elements. At the common interface $\Gamma_{i}=\Gamma_{i}^{e}=\Gamma_{i}^{g}$, the continuity of the field variables is imposed

$$
\begin{array}{ccc}
\text { pressure continuity : } & p_{e}(\mathbf{r})=p_{i}(\mathbf{r})=p_{g}(\mathbf{r}), & \forall \mathbf{r} \in \Gamma_{i}, \\
\text { velocity continuity : } & \mathbf{n}_{e}^{T} \mathbf{v}_{e}(\mathbf{r})=v_{n i}(\mathbf{r})=-\mathbf{n}_{g}^{T} \mathbf{v}_{g}(\mathbf{r}), & \forall \mathbf{r} \in \Gamma_{i},
\end{array}
$$

where the subscript ${ }_{e}, g$ and ${ }_{i}$ refer to the two adjacent elements $\Omega^{e}$ and $\Omega^{g}$ and their common interface $\Gamma_{i}$, respectively.

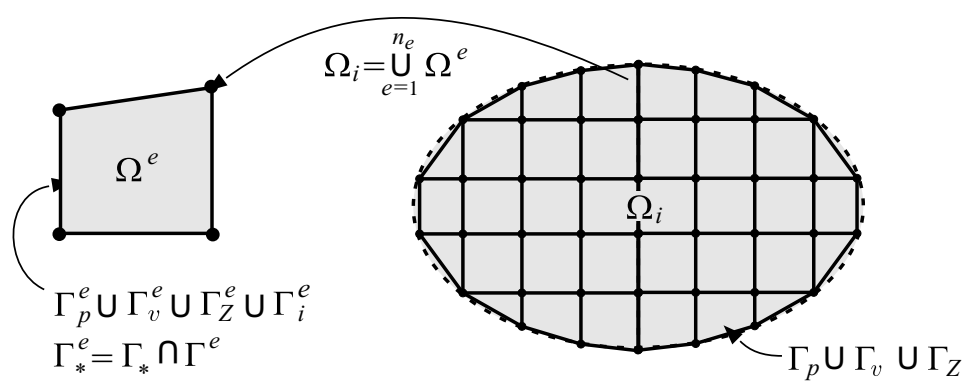

Figure 3: Finite element model for interior acoustic problem

2. Within each element $\Omega^{e}$, a linear combination of polynomial basis functions approximates the exact solution

$$
\begin{aligned}
& p(\mathbf{r}) \approx \hat{p}(\mathbf{r})=\sum_{a=1}^{n_{a}} N_{a}(\mathbf{r}) p_{a}^{e}=\quad \mathbf{N}(\mathbf{r}) \mathbf{p}^{e}, \forall \mathbf{r} \in \Omega^{e}, \\
& \mathbf{v}(\mathbf{r}) \approx \hat{\mathbf{v}}(\mathbf{r})=\frac{j}{\rho \omega} \boldsymbol{\nabla} \hat{p}(\mathbf{r}) \quad=\frac{j}{\rho \omega} \boldsymbol{\nabla} \mathbf{N}(\mathbf{r}) \mathbf{p}^{e}, \forall \mathbf{r} \in \Omega^{e} .
\end{aligned}
$$

The contribution factors $p_{a}^{e}$, stored in the element vector $\mathbf{p}^{e}$, form the unknown element degrees of freedom. These unknown degrees of freedom are generally nodal pressure values. The corresponding basis functions $N_{a}$ are stored in the row vector $\mathbf{N}$.

3. The approximation $(\hat{p}, \hat{\mathbf{v}})$ satisfies the equation of motion (1b) and the pressure continuity in (12a) by definition. Furthermore, the prescribed pressure distribution $\bar{p}(\mathbf{r}) \quad \forall \mathbf{r} \in \Gamma_{p}$ is usually represented by piecewise (bi)linear functions, which can exactly be described by the pressure approximation $\hat{p}$. However, the approximation does not satisfy the remaining equations, i.e. the conservation of mass (1a), the velocity boundary condition in (3b), the impedance boundary condition in (3c) and the velocity continuity in $(12 \mathrm{~b})$. The involved residuals on these relations are enforced to zero in some integral sense. This is achieved by either the weighted residual approach, the principle of virtual work or a variational principle. All three approaches yield the 
following weak problem formulation on element level

$$
\begin{array}{r}
-\omega^{2} \int_{\Omega^{e}} u \frac{1}{c^{2}} \hat{p} \mathrm{~d} \Omega+j \omega \int_{\Gamma_{Z}^{e}} u \frac{\rho}{\bar{Z}} \hat{p} \mathrm{~d} \Gamma+\int_{\Omega^{e}}(\nabla u)^{T}(\nabla \hat{p}) \mathrm{d} \Omega=\ldots \\
\ldots=j \omega \int_{\Omega^{e}} u \rho q \delta\left(\mathbf{r}, \mathbf{r}_{q}\right) \mathrm{d} \Omega-j \omega \int_{\Gamma_{v}^{e}} u \rho \bar{v}_{n} \mathrm{~d} \Gamma-j \omega \int_{\Gamma_{i}^{e}} u \rho v_{n i} \mathrm{~d} \Gamma,
\end{array}
$$

where $u=u(\mathbf{r})$ represents an arbitrary test function, which is zero along $\Gamma_{p}^{e}$.

4. The above weak problem formulation is transformed into a set of $n_{a}$ algebraic equations by following the Galerkin approach. Each basis function $N_{a}$ of the pressure approximation $\hat{p}$ in equation (13a) is selected as test function once. Together with the substitution of the pressure approximation $\hat{p}$, this approach yields the following FE description

$$
\left(-\omega^{2} \mathbf{M}^{e}+j \omega \mathbf{C}^{e}+\mathbf{K}^{e}\right) \mathbf{p}^{e}=j \omega\left(\mathbf{q}^{e}-\mathbf{v}^{e}-\mathbf{v}_{i}^{e}\right) .
$$

In this algebraic equation, $\mathbf{M}^{e}, \mathbf{C}^{e}$ and $\mathbf{K}^{e}$ represent the acoustic mass, damping and stiffness matrix. The vectors $\mathbf{q}^{e}, \mathbf{v}^{e}$ and $\mathbf{v}_{i}^{e}$ are the acoustic loading vectors associated with the point source $q$, the prescribed normal velocity $\bar{v}_{n}$ and the interface velocity $v_{n i}$.

5. Finally, the assembly of all element models results in the following FE model

$$
\left(-\omega^{2} \mathbf{M}+j \omega \mathbf{C}+\mathbf{K}\right) \mathbf{p}=j \omega(\mathbf{q}-\mathbf{v})
$$

where the vector $\mathbf{p}$ is the solution vector. It collects all degrees of freedom, which are nodal pressure values. The pressure boundary conditions are taken into account by assigning the prescribed pressure values directly to the nodal degrees of freedom on the boundary $\Gamma_{p}$, such that these degrees of freedom are no longer unknowns of the FE model. Note that the $\mathbf{v}_{i}^{e}$ contributions of two adjacent elements cancel out each other, because the inter-element pressure continuity along their common interface $\Gamma_{i}^{e}$ is satisfied a priori (conforming elements).

\subsection{Treatment of unbounded domains}

A difficulty in the application of the FEM for exterior acoustic problems lies in the effective treatment of unbounded domains, because these cannot be subdivided into a finite number of elements. Therefore, an artificial boundary $\Gamma_{t}$ is introduced in order to truncate the unbounded domain [27]. This results in a bounded domain $\bar{\Omega}_{e}$ for the exterior acoustic problem, which is subdivided using conventional finite elements (see figure 4). The artificial truncation boundary $\Gamma_{t}$, however, requires some special treatment in order to prevent or to reduce the spurious reflections of waves. The three most commonly used treatments are $[15,16,28]$

1. the application of absorbing boundary conditions,

2. the explicit modelling of the unbounded domain using infinite elements or

3. the introduction of absorbing layers,

which are discussed briefly here. 


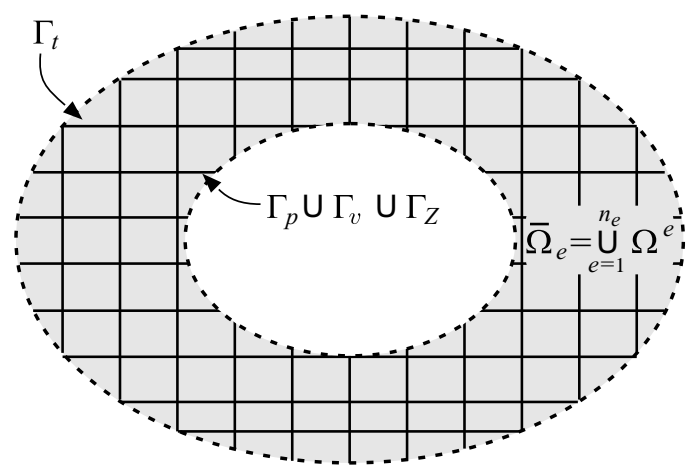

Figure 4: Finite element model for exterior acoustic problem with artificial truncation boundary $\Gamma_{t}$

\subsubsection{Absorbing boundary conditions}

The absorbing boundary conditions are mathematical relations between the pressure $p$ and its derivatives at the truncation boundary $\Gamma_{t}$. A distinction is made between conditions defined by a local scheme and those defined by a global scheme.

The local schemes retain the sparse and banded structure of the FE system matrices. They are applicable to more generally shaped artificial boundaries and they usually guarantee the uniqueness of the solution. The low-order local schemes are easy to implement but they tend to lose accuracy for the higher frequencies [29]. Furthermore, the artificial boundary must be placed far enough from the radiating or scattering object to prevent spurious reflections. The impedance boundary condition in equation (3c) is an example of the most simple, first-order local boundary condition of this type. Higher-order local schemes improve the accuracy but their implementation is more demanding due to the presence of the high-order derivatives. Alternatively, the order of the absorbing boundary conditions can be increased rather easily by the introduction of auxiliary variables which eliminate the high-order derivatives at the expense of additional degrees of freedom [30].

Also in case of the global scheme, the order of boundary conditions can be increased easily to obtain the desired level of accuracy. However, the geometry of truncation boundary $\Gamma_{t}$ is limited to relatively simple shapes. Furthermore, all degrees of freedom on $\Gamma_{t}$ are coupled, which results in a significant reduction of the sparsity of the system and which requires special procedures for implementation and parallelization. The Dirichlet-to-Neumann mapping is the most commonly used approach of this type [31, 32, 33]. The order of the series expansion of the Dirichlet-to-Neumann boundary condition determines the accuracy of the procedure. Despite the theoretical correctness of the approach, the fact of being a global method degenerating the sparse structure of the FE system matrices is a major practical disadvantage.

\subsubsection{Infinite elements}

The truncation boundary $\Gamma_{t}$ is subdivided in a finite number of infinite elements [34]. These elements explicitly model the unbounded domain outside bounded domain $\bar{\Omega}_{e}$. They have finite dimensions in the plane of the truncation boundary $\Gamma_{t}$, but they extend to infinity in the outward normal direction $\mathbf{n}$. The FE approximation in that direction consists of a combination of a suitable amplitude decay and a wave-like radial variation for modelling the outgoing travelling waves. The accuracy of the approximation improves for an increasing radial order of the $\mathrm{FE}$ basis functions, but also the computational costs increase in that 
case.

Two leading approaches have emerged within the infinite element world [35], namely (i) the unconjugated Burnett elements and (ii) the Astley-Leis wave envelope elements. The unconjugated Burnett elements [36, 37] use the same basis functions for both the trial and the test functions (Galerkin approach). This results in symmetric but frequency dependent system matrices. Their condition number increases rapidly for an increasing radial order. Furthermore, the model generation involves some tedious numerical integration. The unconjugated Burnett elements are believed to provide the best accuracy for the near-field whereas the Astley-Leis wave envelope elements are most effective in the far-field [29]. In the wave envelope elements $[38,39]$, the test functions are conjugates of the basis functions in the FE approximation and a geometrical weighting factor is introduced. This results in frequency independent system matrices which are no longer symmetric. Furthermore, the numerical integration is less expensive than for the unconjugated elements and the ill-conditioning can be avoided by the selection of a suitable radial basis. Unfortunately, the performance of both the conjugated and unconjugated infinite elements deteriorates at higher frequencies and for stretched truncation boundaries [40].

\subsubsection{Absorbing layers}

Berenger [41] introduces the perfectly matched layer concept for treating the truncation boundary in exterior electromagnetic wave problems. This concept is applicable for acoustic radiation and scattering problems too [42]. The basic idea is to introduce an exterior layer of finite thickness at the truncation boundary. The purpose of this additional layer is to absorb the outgoing waves. The reflection of plane waves with arbitrary angles of incidence can be eliminated theoretically by splitting a scalar field and by selecting the proper perfectly matched layer coefficients. Perfect absorbance can be reached for increasing layer thickness. All in all, the practical implementation involves several parameters, such as the layer thickness, the number of divisions of the perfectly matched layers and the variation and the limits of their coefficients. Current research focuses on the optimization of these parameters.

\subsection{Accuracy at high frequencies}

The convergence of the FEM is guaranteed if the approximation solution satisfies the following convergence criteria [3].

1. A 'rigid' body mode, i.e. a constant pressure distribution, does not result in a contribution to the particle velocity field.

2. The approximation solution must be able to describe a constant particle velocity field. This criterion is commonly referred to as the criterion of completeness.

3. The particle velocity approximation must be finite at the element boundaries, which implies the imposition of $C_{0}$ continuity conditions for the pressure approximation. This criterion is commonly referred to as the compatibility condition.

The above criteria are satisfied if the 'serendipity' family of $2 \mathrm{D}$ rectangular and 3D brick elements is used or the family of $2 \mathrm{D}$ triangular and $3 \mathrm{D}$ tetrahedral elements. These elements may be distorted in order to mesh the bounded acoustic domain $\Omega_{i}$ or $\bar{\Omega}_{e}$ with an irregularly shaped boundary $\Gamma_{f}\left(\cup \Gamma_{t}\right)$ using less elements. In those cases, the application of the isoparametric mapping procedure ensures that the convergence criteria are still satisfied.

In case of elliptic problems, such as the Poisson problem, the FEM provides an accurate approximation irrespective of the applied mesh size $h$. However, the linear Helmholtz equation is indefinite. The FEM, applied to indefinite problems, converges only if the mesh 
size $h$ is smaller than a critical mesh size $h_{\mathrm{cr}}$. The solution of a time-harmonic acoustic problem contains harmonic components with wavelengths of $\lambda=2 \pi / k=2 \pi c / \omega$. At least two elements are required per wavelength. Consequently, the critical mesh size would be $h_{\mathrm{cr}}=\pi / k=\pi c / \omega$.

In order to describe the spatial variation of the harmonic component with a certain level of accuracy, often the 'rule of thumb' is applied, which states that at least 6 to 10 linear elements should be used per wavelength $\lambda$. Thus, the maximal mesh size according to this rule would be $h_{\max }=1 / k=c / \omega(\approx 6$ elements per $\lambda)$. The 'rule of thumb' controls the approximation error well for low frequencies or small wave numbers, where the approximation error is governed mainly by the interpolation error. The latter error is the difference between the exact solution and the approximation solution, which is based on the linear combination of basis functions weighted by the exact nodal solutions. However, for higher frequencies or increasing wave numbers, the interpolation error gets polluted. That is, the approximation error is no longer governed by the interpolation error only, but the so-called pollution effect gains importance. The FE approximation introduces artificial dispersion, which causes this pollution effect.

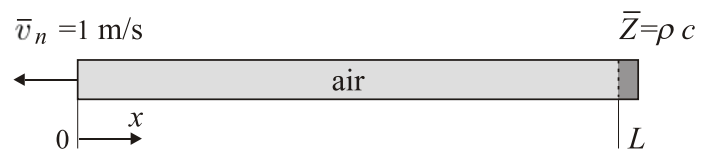

Figure 5: 1D acoustic problem: tube

The consideration of a 1D acoustic problem helps to clarify the distinction between the two sources of error. Figure 5 depicts the $1 \mathrm{D}$ acoustic problem consisting of a tube of length $L$ filled with air $\left(\rho=1.225 \mathrm{~kg} / \mathrm{m}^{3}, c=340 \mathrm{~m} / \mathrm{s}\right)$. A unit normal velocity excites the acoustic system at left end. At the right end, an absorbing boundary condition is imposed, such that the pressure response consists of a propagating wave. The mathematical model is reformulated in the following non-dimensional variables

$$
\tilde{x}=\frac{x}{L}, \tilde{k}=k L \text { and } \tilde{h}=\frac{h}{L} .
$$

This allows one to distinguish between the low wave number range $(\tilde{k} \ll 1)$ and the high wave number range $(\tilde{k}>1)$. Figure 6 shows the pressure distribution for the 'rule of thumb' with $k h=\tilde{k} \tilde{h}=1$ for the high wave number of $\tilde{k}=10$. The approximation shows a wave that propagates with a larger wavelength than the exact solution. In other words, the numerical wave number $\tilde{k}_{n}$ is smaller than the physical wave number $\tilde{k}$, which corresponds to the dispersion-free propagating wave. The erroneous approximation of the relation between the radial frequency $\omega$ and the wave number $\tilde{k}$ is referred to as the dispersion error. The dispersion error causes the pollution effect, which can be defined as the difference between the approximation solution $\hat{p}_{\text {intp }}$, obtained by interpolation of the exact solution $p$, and the FE approximation $\hat{p}_{\mathrm{FE}}$ as clarified in figure 6 . In case of a regular mesh, the dispersion error can be predicted. Consider the patch of elements surrounding node $a$ in a 1D mesh as shown in figure 7. A FE discretization is made of the 1D homogeneous Helmholtz equation based on the linear elements in the patch with the basis functions $N_{a-1}, N_{a}$ and $N_{a+1}$. This results in the following algebraic equation for node $a$

$$
\begin{aligned}
A_{1} p_{a-1}+2 A_{0} p_{a}+A_{1} p_{a+1}=0 \quad \text { with } \quad A_{0}=1-\frac{1}{3}(\tilde{k} \tilde{h})^{2} \\
\text { and } \quad A_{1}=-1-\frac{1}{6}(\tilde{k} \tilde{h})^{2}
\end{aligned}
$$




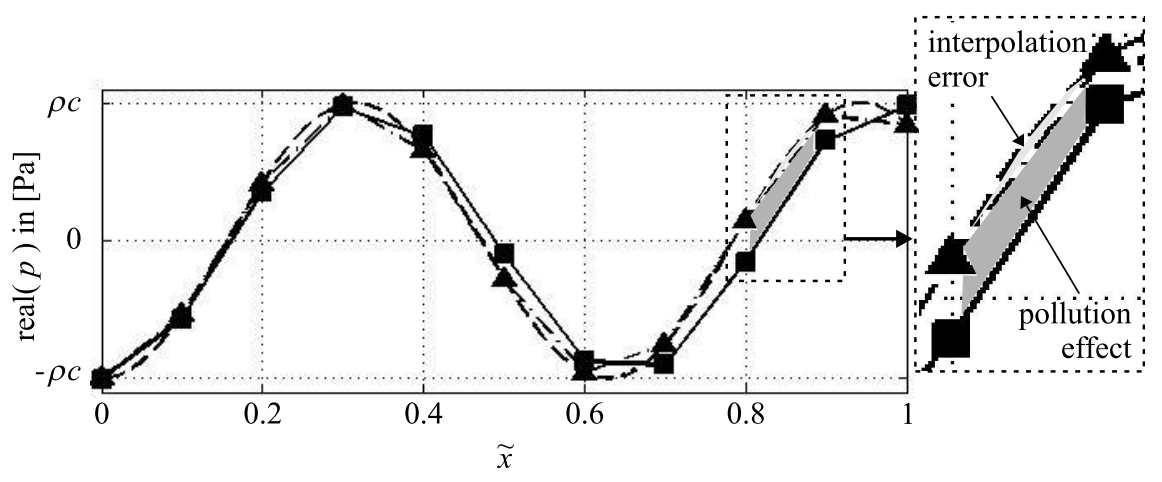

Figure 6: Pressure distribution in the tube at $\tilde{k} \tilde{h}=1\left(--p, \mathbf{\Delta}^{-} \cdot \hat{p}_{\mathrm{intp}}, \mathbf{-}-\hat{p}_{\mathrm{FE}}\right)$
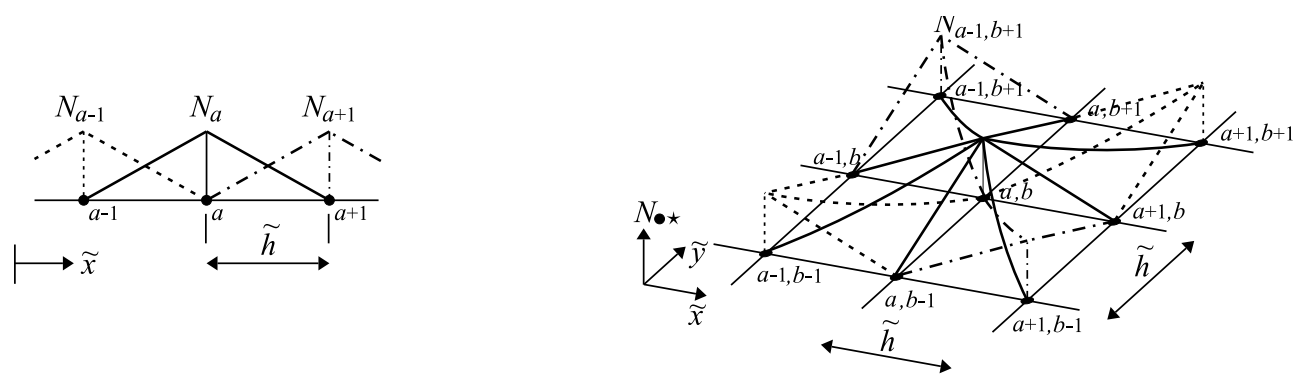

Figure 7: Element patch in regular finite element meshes for dispersion analyses

where $p_{a}$ represent the nodal pressure at position $\tilde{x}_{a}=a \tilde{h}$. The solution of this equation is a propagating wave

$$
p_{a}=\hat{p}\left(\tilde{x}_{a}\right)=\mathrm{e}^{j \tilde{k}_{n} \tilde{x}_{a}}
$$

with the numerical wave number $\tilde{k}_{n}$. The substitution of this solution in the homogeneous differential equation (18) and some further mathematical operations result in the following explicit dispersion relation for the numerical wave number $\tilde{k}_{n}$ multiplied by the mesh size $\tilde{h}$

$$
\tilde{k}_{n} \tilde{h}=\arccos \left(-A_{0} / A_{1}\right) \approx \tilde{k} \tilde{h}-\frac{1}{24}(\tilde{k} \tilde{h})^{3}+\mathcal{O}\left((\tilde{k} \tilde{h})^{5}\right) \quad \text { (Taylor expansion) }
$$

This relation shows (i) that the numerical wave number $\tilde{k}_{n}$ is smaller than the physical wave number $\tilde{k}$ and (ii) that keeping $\tilde{k} \tilde{h}=k h$ constant, as in case of the 'rule of thumb', does not control the dispersion error.

The pollution effect, caused by the dispersion error, is kept within acceptable limits by the following rule

$$
\tilde{k}^{3} \tilde{h}^{2}=\text { constant }
$$

This rule follows from an a priori error estimation discussed by Ihlenburg [4].

A similar dispersion analysis as presented above can be performed for a regular $2 \mathrm{D}$ mesh. Consider the patch of elements surrounding the node at position $\left(\tilde{x}_{a}, \tilde{y}_{b}\right)=(a \tilde{h}, b \tilde{h})$ in a $2 \mathrm{D}$ mesh as shown in figure 7 . The FE discretization, based on bilinear elements with the basis functions $N_{\bullet \star}$, of the $2 \mathrm{D}$ homogeneous Helmholtz equation results in the following 
algebraic equation

$$
\begin{array}{r}
A_{2} p_{a-1, b-1}+A_{1} p_{a, b-1}+A_{2} p_{a+1, b-1}+\ldots \\
\ldots+A_{1} p_{a-1, b}+A_{0} p_{a, b}+A_{1} p_{a+1, b}+\ldots
\end{array} \text { with }\left\{\begin{array}{l}
A_{2} p_{a-1, b+1}+A_{1} p_{a, b+1}+A_{2} p_{a+1, b+1}=0 \\
A_{1}=-\frac{1}{3}-\frac{4}{36}(\tilde{k} \tilde{h})^{2} \\
A_{2}=-\frac{1}{3}-\frac{1}{36}(\tilde{k} \tilde{h})^{2}
\end{array}\right.
$$

where $p_{a, b}$ represents the nodal pressure at position $\left(\tilde{x}_{a}, \tilde{y}_{b}\right)$. The solution of this equation is formed by the set of all propagating waves given by

$$
p_{a, b}=\hat{p}\left(\tilde{x}_{a}, \tilde{y}_{b}\right)=\mathrm{e}^{j \tilde{k}_{n}\left(\tilde{x}_{a} \cos (\theta)+\tilde{y}_{b} \sin (\theta)\right)}
$$

where $\theta$ represents the direction of the propagating wave. The substitution of this solution in the homogeneous differential equation (22) and some further mathematical operations result in the following implicit dispersion relation for the numerical wave number $\tilde{k}_{n}$ multiplied by the mesh size $\tilde{h}$

$$
\begin{aligned}
& A_{0}+2 A_{1}\left(\cos \left(\tilde{\xi}_{1}\right)+\cos \left(\tilde{\xi}_{2}\right)\right)+4 A_{2}\left(\cos \left(\tilde{\xi}_{1}\right) \cos \left(\tilde{\xi}_{2}\right)\right)=0 \quad \text { with } \quad \tilde{\xi}_{1}=\tilde{k}_{n} \tilde{h} \cos (\theta) \\
& \text { and } \quad \tilde{\xi}_{2}=\tilde{k}_{n} \tilde{h} \sin (\theta) \text {. }
\end{aligned}
$$

Figure 8 visualizes this relation for the 'rule of thumb' with $\tilde{k} \tilde{h}=1$. The numerical wave number $\tilde{k}_{n}$ is clearly direction dependent and smaller than the physical wave number $\tilde{k}$ for each direction $\theta$. The largest differences occur for waves propagating along the grid lines, which equals the difference between the numerical wave number $\tilde{k}_{n}$ and the physical $\tilde{k}$ in the $1 \mathrm{D}$ case. Therefore, rule $(21)$, where $\tilde{k}^{3} \tilde{h}^{2}$ is kept constant, will control the pollution effect for the $2 \mathrm{D}$ case too. The extension to the $3 \mathrm{D}$ case is straightforward and, therefore, it is not considered here.

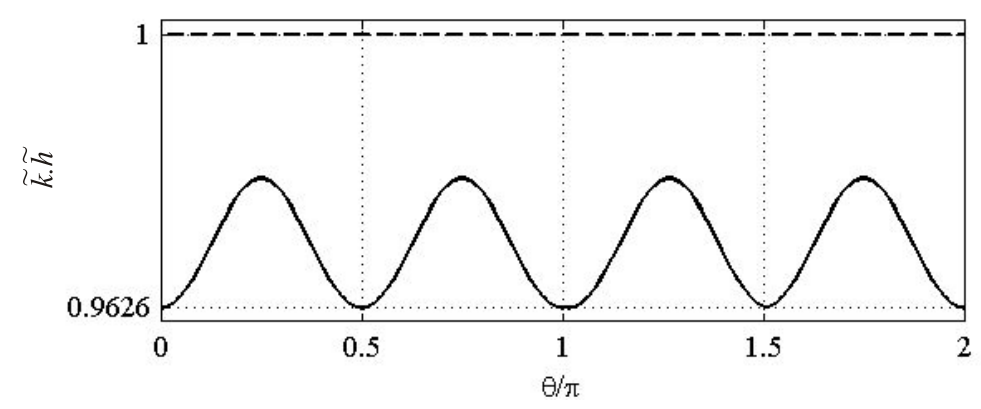

Figure 8: Dispersion curve for $\tilde{k} \tilde{h}=1\left(--\tilde{k},-\tilde{k}_{n}\right)$

The simple dispersion analyses presented here illustrate the challenge in high-frequency acoustics. The convergence of the FEM applied to time-harmonic acoustics is mesh size dependent. A 'rule of thumb' $\left(k h_{\max }=(\omega / c) h_{\max }=1\right)$ states that at least 6 to 10 linear elements should be used per wavelength $\lambda$ to control the interpolation errors. Since the wavelength $\lambda=2 \pi c / \omega$ is inversely proportional to the radial frequency $\omega$, the number of elements needs to grow for increasing frequencies in order to comply with the 'rule of thumb'. Moreover, at high frequencies, the FE approximation shows artificial dispersion which pollutes the interpolation errors. The more stringent rule (21) is necessary to control these pollution effects resulting in a further increase of the number of degrees of freedom. 
Both the mesh size dependent convergence and the numerically introduced dispersion complicate the application of the classical FEM for high-frequency acoustics. Therefore, in order to meet this computational challenge, the enhancements and the extensions of the FEM focus on reducing these two main drawbacks. However, the 2D dispersion analysis shows already that this will not be easy due to the direction dependency of the numerically introduced dispersion.

More details on the numerical dispersion analysis and on the error estimation in the FEM applied to linear acoustics can be found in [4]. This reference also considers the $p$ version of the FEM, which uses higher order polynomial basis functions and thereby suffers less from numerical dispersion.

\subsection{Enhancements and extensions}

Several enhancements and extensions of the FEM coexist which try to improve the accuracy at high frequencies. Their number is too large to discuss them all in detail. Nevertheless, this paper tries to capture most of them in five categories, which are

1. the process optimization methods,

2. the domain decomposition methods,

3. the stabilized methods,

4. the generalized methods and

5. the multi-scale methods.

The remaining part of this chapter gives a brief overview of the state-of-the-art per category.

\subsubsection{Process optimization methods}

Several FE processes can be optimized. The computational resources, which come available in this way, are used to fulfil the mesh size criteria at higher frequencies. The most obvious optimization techniques are the $r$-, $h$ - and $p$-refinement and their combinations (see a.o. $[43,44,45,46,47])$. They usually require a posteriori error estimators for which Ainsworth and Oden [48] and Zienkiewicz [49] give general overviews. Oden et al. [50] focus more on their application in acoustics. An adaptive approach improves the efficiency further by refining the regions with largest errors more.

Another process, which can be optimized, is the FE model generation. This process includes a large number of integral evaluations, which are usually performed numerically. A reduced numerical integration scheme obviously results in a cost reduction. However, this cost reduction is only marginal since the FE model generation is responsible for only a small part of the overall computational costs. The major advantage of reduced integration is its positive influence on the numerical dispersion. Thompson and Kunthong [51] and Guddati and Yue [52], for example, have recognized this effect and they make even a more efficient use of it by selecting non-conventional integration point positions.

Solving the system of algebraic equations consumes most of the computing time. These systems consist of huge but sparsely populated matrices due to the mesh requirements at high frequencies. The classical Gaussian elimination and factorization schemes are not applicable anymore. The use of skyline solvers is inevitable. Direct skyline solvers are available [53], but the sparse iterative solvers become more and more standard in large scale numerical analyses [54]. These iterative solvers are computational more efficient, but they lack robustness and their performance is highly problem dependent. However, the mathematical community continuously improves the iterative solvers and makes them generally applicable. 


\subsubsection{Domain decomposition methods}

Despite the continuous growth of the available computational capacity and despite the improvement of the sparse iterative solvers, the FE models may still be too large for reallife acoustic problems in e.g. automotive or aerospace engineering. Domain decomposition methods allow one to tackle these problems based on a divide and conquer principle. That is, it is more efficient to solve a large number of small problems than to solve a single huge problem. An additional advantage of this strategy is that it is well suited for parallel implementation.

The component mode synthesis [55] is a well established tool in the field of structural dynamics. It consist of subdividing a structure into several components. The component FE models are reduced using a small basis of Ritz vectors. The reduced models are assembled and solved for the overall reduced set of degrees of freedom. The Craig-Bampton method is probably the most popular component mode synthesis method. It requires a.o. a Ritz vector for each FE node on the common interface between components. This is disadvantageous for the acoustic problems considered here because (i) the interfaces are not discrete coupling points but lines (2D) or surfaces (3D) and (ii) the number of interface nodes grows with frequency in order to satisfy the mesh requirements.

This drawback of the component mode synthesis is overcome by the automated multilevel substructuring method $[56,57]$. It follows a procedure which automatically subdivides the FE model (i) in a large number of substructures (ii) on multiple levels (iii) based on the sparsity of the model matrices. A large number of small eigenvalues needs to be solved in this way. Consequently, this multi-level method is computational far more efficient than the component mode synthesis and it is inherently better suited for parallelization. Kropp and Heiserer [58] and Stryczek et al. [59] have applied this method successfully for solving real-life structural-acoustic problems in automotive engineering.

Schwartz alternating procedures incorporate the domain decomposition principles directly in the sparse iterative solvers $[54,60,61]$. The problem domain is subdivided into (non-)overlapping subdomains. Schwartz alternating procedures follow a two-level strategy to solve the corresponding subproblems. On the local level, each subproblem is solved using the intermediate pressure solutions of neighbouring subdomains as essential boundary conditions. On the global level, a reduced problem is solved iteratively for the unknown interface degrees of freedom.

The Schwartz alternating procedures have been applied successfully in many engineering disciplines, but the indefiniteness of the Helmholtz equation poses serious challenges on their performance [62]. Instead of enforcing essential boundary conditions on the local level, Farhat and Roux [63] propose the use of the Lagrange multiplier technique to interconnect the non-overlapping subdomains. This results in the so-called finite element tearing and interconnecting method. The overall problem is described by the reduced set of Lagrange multipliers and solved iteratively on the global level. The method has proven to provide reliable results if (i) proper Lagrange multipliers are selected, which regularize the local problems, and if (ii) the global problem is preconditioned properly. The finite element tearing and interconnecting method has been applied successfully a.o. in acoustics $[62,64$, $65,66]$ and in structural-acoustics [67].

\subsubsection{Stabilized methods}

The stabilized FE methods belong to the first class of methods which aim at reducing the artificial dispersion. They modify the weak problem formulation for that purpose such that it becomes unconditionally stable [68]. This is achieved by including additional residual terms in the bilinear part $a(u, \hat{p})$ of the weak problem formulation in equation (14), which 
is given by

$$
a(u, \hat{p})=-\omega^{2} \int_{\Omega^{e}} u \frac{1}{c^{2}} \hat{p} \mathrm{~d} \Omega+j \omega \int_{\Gamma_{Z}^{e}} u \frac{\rho}{\bar{Z}} \hat{p} \mathrm{~d} \Gamma+\int_{\Omega^{e}}(\nabla u)^{T}(\nabla \hat{p}) \mathrm{d} \Omega .
$$

For example, the Galerkin least-squares (GLS) method [69, 70] includes the residual on the Helmholtz equation in this way and the Galerkin gradient least-squares (GVLS) method $[71,72]$ the gradient of this residual

$$
\begin{gathered}
a_{\mathrm{GLS}}(u, \hat{p})=a(u, \hat{p})+\int_{\Omega^{e}}\left(\Delta u-k^{2} u\right) \tau\left(\Delta \hat{p}+k^{2} \hat{p}+j \rho \omega q \delta\left(\mathbf{r}, \mathbf{r}_{q}\right)\right) \mathrm{d} \Omega, \\
a_{\mathrm{G} \nabla \mathrm{LS}}(u, \hat{p})=a(u, \hat{p})+\int_{\Omega^{e}}\left(\nabla\left(\Delta u-k^{2} u\right)\right)^{T} \tau \nabla\left(\Delta \hat{p}+k^{2} \hat{p}+j \rho \omega q \delta\left(\mathbf{r}, \mathbf{r}_{q}\right)\right) \mathrm{d} \Omega,
\end{gathered}
$$

where $\tau$ represents an algebraic design parameter. These additional residual terms result in a modification of the model matrix $\left(-\omega^{2} \mathbf{M}+j \omega \mathbf{C}+\mathbf{K}\right)$ of the FE model in equation (16). The optimal design parameter $\tau_{\text {opt }}$ follows from a dispersion analysis. The selection of $\tau_{\text {opt }}$ eliminates the dispersion error completely in the 1D case. Unfortunately, this is not the case for higher-dimensional problems. The selection of $\tau_{\text {opt }}$ can only eliminate the dispersion error in certain preferred directions. If the exact solution does not include large contributions of wave components in those preferred directions, then the application of this type of stabilization scheme has less influence on the overall accuracy.

The quasi-stabilized method [73, 74], on the other hand, minimizes the dispersion error over all directions. This is achieved by modifying the coefficients of the dispersion relation, i.e. $A_{0}, A_{1}$ and $A_{2}$ in equations (18) and (22). Numerical experiments on the 2D Helmholtz equation with a regular FE mesh illustrate that the quasi-stabilized method outperforms the Galerkin least-squares method.

The local mesh-dependent augmented Galerkin method [75] is even more enhanced in the sense that it considers the numerical dispersion as a position dependent acoustic property. An artificial anisotropic dispersion is introduced to suppress the numerical dispersion and thereby restoring the exact propagation characteristics. The method outperforms the Galerkin least-squares method applied to 2D acoustic problems with non-uniform rectangular meshes. However, further research is needed to extend the method for arbitrarily distorted meshes.

\subsubsection{Generalized methods}

The generalized methods are based on the partition-of-unity method [76, 77]. They aim at reducing the artificial dispersion by incorporating a priori knowledge about the global, shortwavelength behaviour in the local approximation field. The application of these new approximations in the $\mathrm{FE}$ framework results in a modified model matrix $\left(-\omega^{2} \mathbf{M}+j \omega \mathbf{C}+\mathbf{K}\right)$ of the FE model in equation (16).

In case of the partition-of-unity FEM [78, 79, 80, 81], a priori knowledge is incorporated by replacing the local approximation in equation (13a) by a linear combination of free-space solutions $\Phi_{b}(\mathbf{r})$ multiplied by the conventional polynomial basis functions $N_{a}(\mathbf{r})$

$$
\hat{p}(\mathbf{r})=\sum_{a=1}^{n_{a}} N_{a}(\mathbf{r}) p_{a}^{e} \rightarrow \hat{p}(\mathbf{r})=\sum_{a=1}^{n_{a}} N_{a}(\mathbf{r}) \sum_{b=1}^{n_{b}(a)} \Phi_{b}(\mathbf{r}) q_{b a}^{e} \quad \forall \mathbf{r} \in \Omega,
$$

where $q_{b a}^{e}$ represent the unknown degrees of freedom, which are generally not nodal pressure values. The use of the basis functions $N_{a}(\mathbf{r})$ localizes the global, short-wavelength behaviour 
and it guarantees the inter-element pressure continuity. The free-space solutions, which describe the short-wavelength behaviour, have been selected from the set of

$$
\begin{array}{rll}
\text { generalized harmonic polynomials: } & \mathrm{e}^{ \pm j n \theta} J_{n}(k r), & n=0,1,2, \ldots \\
\text { and plane wave functions: } & \mathrm{e}^{j k r \cos \left(\theta-\theta_{n}\right)}, & n=1,2, \ldots \\
& \theta_{n} \in[0,2 \pi] .
\end{array}
$$

In these relations, $\mathbf{r}=(r, \theta)$ represents the polar coordinate and $J_{n}$ the Bessel functions of the first kind. The application of the latter set of plane wave functions for the $2 \mathrm{D}$ Helmholtz equation with a regular FE mesh shows an improved computational efficiency compared with the stabilized methods. However, the treatise of essential boundary conditions and the numerical integration require special attention. Furthermore, the set of algebraic equations becomes ill-conditioned for a large number of free-space solutions $\Phi_{b}(\mathbf{r})$. These computational issues hold generally for all partition-of-unity based methods.

Another member of this class is the element-free Galerkin method [82, 83, 84, 85]. It is based on the moving least-squares method [86] and, therefore, it forms a specific instance of the partition-of-unity [77]. A weighted sum of a number of free-space solutions approximates the pressure in the neighbourhood of the node at position $\mathbf{r}_{i}$

$$
\hat{p}(\mathbf{r})=\sum_{b=1}^{n_{b}} \Phi_{b}(\mathbf{r}) \alpha_{b}(\mathbf{r}), \quad \forall\left\|\mathbf{r}-\mathbf{r}_{i}\right\| \leq D_{i} .
$$

$D_{i}$ represents the domain of influence of node $i$, which is a measure for the size of the neighbourhood surrounding node $i$. The coefficients $\alpha_{b}(\mathbf{r})$ are position dependent too. They are obtained by a least-squares fit of the pressure approximation $\hat{p}$ for all $n_{i}$ nodes, which are scattered over the acoustic domain $\Omega$. The pressure approximation can be expressed as

$$
\hat{p}(\mathbf{r})=\sum_{i=1}^{n_{i}} \Psi_{i}(\mathbf{r}) p_{i}, \quad \forall \mathbf{r} \in \Omega
$$

where $\Psi_{i}(\mathbf{r})$ represents the globally defined basis function associated with node $i$, which contains knowledge about the short-wavelength behaviour, and $p_{i}$ is the corresponding nodal degree of freedom. Numerical experiments show that the level of artificial dispersion obtained with the element-free Galerkin method is comparable with the levels obtained with the quasi-stabilized method.

\subsubsection{Multi-scale methods}

The multi-scale or multi-grid methods aim at reducing the artificial dispersion by incorporating a priori knowledge about the short-wavelength behaviour in the local approximation field similar to the generalized methods. However, the multi-scale methods follow an additive approach rather than the multiplicative approach to do so. References [87, 88] provide a general framework for the multi-scale methods, which is the variational multi-scale method detailed below.

The basic assumption of the variational multi-scale method is that the exact solution of the acoustic problem described by equations (2) and (3) is given by

$$
p(\mathbf{r})=\hat{p}(\mathbf{r})+p^{\prime}(\mathbf{r}), \quad \forall \mathbf{r} \in \Omega .
$$

This relation represents the direct sum of the coarse-scale solution $\hat{p}(\mathbf{r})$, for which the classical FE solution of equation (13a) is chosen, and the fine-scale solution $p^{\prime}(\mathbf{r})$, which 
is solved analytically. The fine-scale solution $p^{\prime}(\mathbf{r})$ describes the global, short-wavelength behaviour. It is defined for element interiors only, i.e. it vanishes on the element boundaries $\cup_{e=1}^{n_{e}} \partial \Omega^{e}$. The weak form of the acoustic problem is decomposed into the following two subproblems

$$
\begin{array}{r}
\text { coarse-scale problem: } a(u, \hat{p})+a\left(u, p^{\prime}\right)=b(u) \\
\text { fine-scale problem: } a\left(u^{\prime}, \hat{p}\right)+a\left(u^{\prime}, p^{\prime}\right)=b\left(u^{\prime}\right)
\end{array}
$$

where $a(v, p)$ and $b(v)$ represent the bilinear and the linear operator, which are given by the following relations in case of the acoustic problem

$$
\begin{aligned}
& a(v, p)=-\omega^{2} \int_{\Omega} v \frac{1}{c^{2}} p \mathrm{~d} \Omega+j \omega \int_{\Gamma_{Z}} v \frac{\rho}{\bar{Z}} p \mathrm{~d} \Gamma+\int_{\Omega}(\nabla v)^{T}(\nabla p) \mathrm{d} \Omega, \\
& b(v)=j \omega \int_{\Omega} v \rho q \delta\left(\mathbf{r}, \mathbf{r}_{q}\right) \mathrm{d} \Omega-j \omega \int_{\Gamma_{v}} v \rho \bar{v}_{n} \mathrm{~d} \Gamma .
\end{aligned}
$$

Furthermore, the arbitrary test function $u^{\prime}=u^{\prime}(\mathbf{r})$ in equation $(32 \mathrm{~b})$ belongs to the same solution space as the fine-scale solution $p^{\prime}(\mathbf{r})$. The fine-scale problem is solved analytically, which results in an expression for the fine-scale solution as function of the coarse-scale residuals $R(\hat{p})$ on the Helmholtz equation and the boundary conditions

$$
p^{\prime}=M^{\prime}(R(\hat{p})),
$$

where $M^{\prime}$ is a non-local integral operator. The substitution of the fine-scale solution in the coarse problem results in the following weak problem formulation for the coarse scales

$$
a(u, \hat{p})+a\left(u, M^{\prime}(R(\hat{p}))\right)=b(u)
$$

which exactly accounts for the non-local effect of the short-wavelength behaviour. Recall that the underlying fine-scale solution vanishes on the element boundaries. Consequently, the weak problem in equation (35) can be expressed on element level resulting in an expression which resembles the weak problem formulation of the stabilized methods. This indicates that the two classes of methods are related.

In general, it is difficult to obtain a closed-form expression for the non-local operator $M^{\prime}$. Therefore, several multi-scale methods have been proposed, which differ in the way they approximate the non-local operator, i.e. how they approximate the fine-scale solution $p^{\prime}(\mathbf{r})$ in equation (32b). In the residual-free bubble method [89, 90], the fine-scale solution consists of a weighted sum of residual-free bubble functions. These bubble functions are solutions of the fine-scale problem associated with the basis functions $N_{a}(\mathbf{r})$ of the FE approximation on the coarse scales.

Since the set of bubble functions needs to be computed for each element, the residualfree bubble methods is computationally demanding. The computational costs are reduced substantially if a set of alternative bubble functions is used, which is computed only once per frequency of interest for a single parent element [91]. These alternative bubble functions are not exact solutions of the fine-scale problem, but they form merely a hierarchical extension of the coarse-scale approximation space. Numerical experiments show that the same level of accuracy is reaches as the FEM but for frequencies which are three times higher.

Oberai and Pinsky [92] solve the fine-scale problem (32b) approximately by application of Green's functions. The derived multi-scale method shows a super convergent behaviour in the $1 \mathrm{D}$ case. However, for a $2 \mathrm{D}$ problem with a plane wave as exact solution, the accuracy 
B. Pluymers, B. van Hal, D. Vandepitte and W. Desmet

depends on the direction of the wave propagation. This deficiency holds for all multi-scale methods based on a fine-scale solution, which vanishes on the element boundaries.

In the discontinuous enrichment method [93, 94], the above restriction on the fine-scale solution is somewhat relaxed. This allows the use of a free-field solution of the Helmholtz equation $\Phi_{b}(\mathbf{r})$ as fine-scale basis functions, similar to the ones used by the generalized methods, which do not vanish on the element boundaries. Consequently, the direct sum of the FE approximation $\hat{p}(\mathbf{r})$ and the fine-scale solution $p^{\prime}(\mathbf{r})$ may violate the pressure continuity across element boundaries (non-conforming elements). The application of the Lagrange multiplier technique enforces the pressure continuity weakly. Numerical experiments show that the discontinuous enrichment methods suffers less from pollution errors than the classical FEM. Furthermore, the involved models are not as ill-conditioned as partition-of-unity FE models of comparable numerical accuracy.

\section{BOUNDARY ELEMENT METHOD}

\subsection{Basic formulations}

The boundary element method (BEM) is a numerical prediction method which is often applied for solving engineering problems [5]. Amongst others, the method is also well established for the solution of acoustic problems [6, 7]. Unlike the FEM, which makes direct use of the Helmholtz differential equation (2), the BEM starts from a boundary integral formulation for constructing a numerical model. Depending on the choice of the integral formulation, (6) or (9), a direct boundary element (BE) or an indirect BE formulation is obtained. This section outlines the basic formulation of the both approaches.

\subsubsection{Direct boundary element method}

The direct BEM is based on the direct boundary integral (6) and, hence, can be used for the solution of interior acoustic problems or exterior acoustic problems with a closed boundary surface $\Gamma_{f}$. The method follows a two-step procedure.

In the first step the pressure and normal velocity distributions on the closed boundary surface $\Gamma_{f}$ are determined. In a similar way as in the FEM, the closed boundary surface $\Gamma_{f}$ is discretized into a number of small subsurfaces $\Gamma^{e}$ such that $\Gamma_{f}=\cup_{e=1}^{n_{e}} \Gamma^{e}$ with $\Gamma^{e} \cap \Gamma^{g}=0$, $\forall e \neq g$ ). These subsurfaces are referred to as boundary elements. Within each boundary element, the distributions of the pressure $p(\mathbf{r})$ and normal velocity $v_{n}(\mathbf{r})$ are approximated by a linear combination of polynomial basis functions

$$
\begin{gathered}
p(\mathbf{r}) \approx \hat{p}(\mathbf{r})=\sum_{a=1}^{n_{a}} N_{a}^{e}(\mathbf{r}) p_{a}^{e}, \quad \forall \mathbf{r} \in \Gamma^{e}, \\
v_{n}(\mathbf{r}) \approx \hat{v_{n}}(\mathbf{r})=\sum_{a=1}^{n_{a}} N_{a}^{e}(\mathbf{r}) v_{n a}^{e}, \quad \forall \mathbf{r} \in \Gamma^{e} .
\end{gathered}
$$

With $n_{a}$ the number of nodes associated with boundary element $\Gamma^{e}$. Based on the element shape functions $N_{a}^{e}$, which are locally defined within one boundary element $\Gamma^{e}$, some global shape functions $N_{a}$ may be constructed, which are defined in the entire boundary surface $\Gamma_{f}$. In this way, global boundary variable expansions may be defined as

$$
\begin{gathered}
p(\mathbf{r}) \approx \hat{p}(\mathbf{r})=\mathbf{N}(\mathbf{r}) \mathbf{p}, \quad \forall \mathbf{r} \in \Gamma_{f}, \\
v_{n}(\mathbf{r}) \approx \hat{v_{n}}(\mathbf{r})=\mathbf{N}(\mathbf{r}) \mathbf{v}_{\mathbf{n}}, \quad \forall \mathbf{r} \in \Gamma_{f} .
\end{gathered}
$$

The contribution factors $p_{a}^{e}$ and $v_{n a}^{e}$, stored in the $\left(n_{b} \times 1\right)$ column vectors $\mathbf{p}$ and $\mathbf{v}_{\mathbf{n}}$, respectively, with $n_{b}$ the total number of boundary surface nodes, form the unknown degrees 
of freedom. The corresponding global basis functions $N_{a}$ are stored in the $\left(1 \times n_{b}\right)$ row vector $\mathbf{N}$. Note that, although this is assumed in equation (36), the shape functions for the expansion of the boundary surface pressure and the boundary surface normal velocity do not have to be the same.

The determination of the unknown degrees of freedom is commonly based on a collocational scheme. In this scheme, the direct boundary integral formulation (6), using the boundary variable expansions (36), is evaluated for positions $\mathbf{r}$, which correspond to the locations of the $n_{b}$ nodes in the boundary surface discretization. This results in the following matrix equation

$$
\mathbf{A} \mathbf{p}=j \rho \omega \mathbf{B} \mathbf{v}_{\mathbf{n}}
$$

with matrices $\mathbf{A}$ and $\mathbf{B}$ the $\left(n_{b} \times n_{b}\right)$ direct BEM system matrices. This linear system of equations has twice as many unknowns as it has equations. Since the boundary conditions (3) impose either a prescribed pressure, a prescribed normal velocity or a prescribed normal impedance relation between both boundary variables, at each node either the pressure value $p_{a}^{e}$ or the normal velocity value $v_{n a}^{e}$ or their normal impedance relation is known a priori. Hence, the number of unknowns reduces with $n_{b}$, and the remaining $n_{b}$ unknown boundary variables may be determined by solution of the system.

In the second, post-processing step, the pressure in any point of the interior or exterior acoustic domain is obtained from the direct boundary integral formulation (6), using the surface results from the first step. In this way, the approximation for the pressure at a certain point $\mathbf{r} \in \Omega_{\{i / e\}} \backslash \Gamma_{f}$ is

$$
p(\mathbf{r})=\mathbf{C} \mathbf{p}+\mathbf{D} \mathbf{v}_{\mathbf{n}}
$$

\subsubsection{Indirect boundary element method}

The indirect BEM is based on the indirect boundary integral (9) and, hence, can be used for the solution of combined interior/exterior acoustic problems or exterior acoustic problems with an open boundary surface $\Gamma_{f}$. Identical the direct BEM, the indirect BEM follows a two-step procedure.

In the first step, the boundary surface variables, being the single and double layer potentials (10), are determined. In a similar way as in the direct BEM, the closed boundary surface $\Gamma_{f}$ is discretized into a number of boundary elements. Within each boundary element, the single and double layer potential $\sigma(\mathbf{r})$ and $\mu(\mathbf{r})$ are approximated by a linear combination of locally defined polynomial basis functions. The construction of global shape functions allows to define the following global boundary variable expansions

$$
\begin{aligned}
& \sigma(\mathbf{r}) \approx \hat{\sigma}(\mathbf{r})=\mathbf{N}(\mathbf{r}) \boldsymbol{\sigma}, \quad \forall \mathbf{r} \in \Gamma_{f}, \\
& \mu(\mathbf{r}) \approx \hat{\mu}(\mathbf{r})=\mathbf{N}(\mathbf{r}) \boldsymbol{\mu}, \quad \forall \mathbf{r} \in \Gamma_{f} .
\end{aligned}
$$

With $\boldsymbol{\sigma}$ and $\boldsymbol{\mu}$ two $\left(n_{b} \times 1\right)$ column vectors, storing the unknown shape function contributions. The $\left(1 \times n_{b}\right)$ row vector $\mathbf{N}$ stores the corresponding global shape functions. Note that, although this is assumed in equation (40), the shape functions for the expansion of the single and double layer potential do not have to be the same.

The use of a collocational approach to determine the unknown degrees of freedom, as selected in the direct BEM, becomes much more difficult, since the evaluation of the indirect boundary formulation (9) for discrete positions $\mathbf{r}$ on the boundary $\Gamma_{f}$ requires the numerical evaluation of Hadamard finite part integrals [26]. Therefore, another solution technique is used, which relies on a variational formulation and which leads to regular integrals. Minimization of the functional involved with the variational formulation results 
in the following matrix equation

$$
\left[\begin{array}{cc}
\mathbf{B} & \mathrm{C} \\
\mathbf{C}^{T} & \mathrm{D}
\end{array}\right]\left\{\begin{array}{l}
\boldsymbol{\sigma} \\
\boldsymbol{\mu}
\end{array}\right\}=\left\{\begin{array}{l}
\mathbf{f}_{\boldsymbol{\sigma}} \\
\mathbf{f}_{\boldsymbol{\mu}}
\end{array}\right\}
$$

with matrices $\mathbf{B}, \mathbf{C}$ and $\mathbf{D}$ the $\left(n_{b} \times n_{b}\right)$ indirect BEM system matrices and with $\mathbf{f}_{\boldsymbol{\sigma}}$ and $\mathbf{f}_{\boldsymbol{\mu}}$ the $\left(n_{b} \times 1\right)$ right-hand side vectors. Since, from the imposed boundary conditions (3), at each node either the single layer potential, the double layer potential or a relation between both boundary variables can be derived a priori, the number of unknowns reduces to $n_{b}$ boundary variables, which may be determined by solution of the system.

In the second, post-processing step, the pressure in any point of the interior or exterior acoustic domain is obtained from the indirect boundary integral formulation (9), using the surface results from the first step. In this way, the approximation for the pressure at a certain point $\mathbf{r} \in \Omega_{\{i / e\}} \backslash \Gamma_{f}$ is

$$
p(\mathbf{r})=\mathbf{A}_{\boldsymbol{\sigma}} \boldsymbol{\sigma}+\mathbf{A}_{\boldsymbol{\mu}} \boldsymbol{\mu} .
$$

\subsection{Comparison with the finite element method}

Since the BEM and the FEM are based on a completely different modelling approach, both methods yield numerical models with very different characteristics. Due to the application of a boundary integral formulation, a BE approach only discretizes the boundary of the considered problem such that the numerical BE models are smaller than corresponding FE models. However, the BE system matrices are fully populated with complex and frequency dependent coefficients and result from more complicated numerical integrations, as compared to the sparsely populated FE system matrices which can be decomposed into a number of frequency independent submatrices whose coefficients result from simple integrations of polynomial functions. Since the boundary integral formulations, applied in the BEM, are available as analytical expressions, there is no additional accuracy decrease for derived acoustic variables, unlike with the FEM, where the polynomial shape functions, which approximate the fundamental acoustic field (most commonly the acoustic pressure), result in polynomial functions of lower order to approximate the higher order derived variables. Furthermore, in contrast with the FEM, the BEM can easily handle problems with unbounded acoustic domains, since the Sommerfeld radiation condition is inherently satisfied. Finally, like the FEM (see section 3.3), the BEM also suffers from numerical pollution. As a result, the $\mathrm{BE}$ discretization also needs to be refined with increasing frequency [95].

It is clear that assembly of a BE model is more time consuming as compared to the fast assembly of a FE model. Whereas in a FE analysis, the bulk part of the computational efforts is spent in solving the system of equations, a major part of the computational efforts in a BE analysis is needed for constructing the model. Depending on the size of the models, it is possible that the solution time for a densely populated BE model is lower that the solution time of an associated, but much larger, sparsely populated FE model. However, despite the possibly lower solution time, the BEM cannot counterbalance the increase in

construction time. In conclusion, the BEM can hardly compete with the FEM for solving interior acoustic problems [96]. However, the BEM does become an efficient alternative for tackling problems in unbounded domains. Nevertheless, the practical use of both the FEM and the BEM is restricted to low-frequency applications.

\subsection{Enhancements and extensions}

A major disadvantage of the BEM is the associated fully populated system matrix. This section briefly discusses some enhancements and extensions of the BEM which pursue decreasing of the density of the matrices, retaining a sparse structure, allowing a faster calcu- 
lation of the coefficients and faster solution of the model. The enhancements and extensions are captured in three categories:

1. the process optimization methods,

2. the generalized methods and

3. the multipole methods.

\subsubsection{Process optimization methods}

In conventional direct BEM, the pressure response is governed by an integral equation, relating the pressure anywhere in the domain to a pressure and normal velocity distribution on the boundary. As a result, the obtained system matrix is fully populated. For acoustic radiation problems the velocity distribution over the boundary is a priori known. Rayleigh methods $[97,98]$ consider the vibrating boundary as a collection of independently vibrating rigid pistons, whose amplitude of vibration is determined by the applied boundary conditions. As a result the pressure in a point located in the acoustic problem domain can be determined as a single integral over the boundary of the piston velocity and an appropriate (singular) source formulation

$$
p(\mathbf{r})=\int_{\Gamma_{f}} v_{n}(\boldsymbol{\xi}) \Upsilon(\mathbf{r}, \boldsymbol{\xi}) \mathrm{d} \Gamma(\boldsymbol{\xi})
$$

with $v_{n}(\boldsymbol{\xi})$ the piston velocity and $\Upsilon(\mathbf{r}, \boldsymbol{\xi})$ the source formulation. Depending on the applied approximation, $\Upsilon$ can be the free-field solution of a point source (7), the solution of a point source in a semi-infinite environment, a collection of free-field solutions taking into account reflections, etcetera. In this way, no large numerical matrix equation is constructed or needs to be solved. However, Rayleigh methods lose accuracy at low frequencies and close to the boundary.

The high-frequency BEM [97] is an alternative approximating method which assumes the radiation impedance at the boundary surface to be approximately the same as the $\rho c$ characteristic impedance of the medium. For response points where $k r$ becomes very large, with $k$ the wave number and $r$ the distance to the boundary, the high-frequency BEM develops into a Rayleigh method.

The lumped parameter BEM [99, 100] is a BEM which offers significant gains both in assembly and solution speed. The concept of the method is to approximate the domain boundary conditions in a special way. Both the normal velocity field and the pressure field are characterized by a single lumped parameter for each discrete boundary element. The physical basis for this approach is given by Fahnline and Koopman [101]. The lumped parameter BEM applies a dual set of shape functions, namely higher-order functions to describe the geometry and constant functions to approximate the boundary variables.

\subsubsection{Generalized methods}

Generalized BEMs incorporate a priori known information into the formulation, comparable to the generalized FEMs (see section 3.4.4).

Perrey-Debain et al. [102] and Perrey-Debain et al. [103] developed an extension to the conventional direct BEM which is called the wave boundary element method. Using ideas arising from the partition-of-unity FEM, the underlying wave behaviour of the solution is incorporated into the formulation of the boundary elements. As a result each element may span a larger area of the boundary than a conventional BE. The wave boundary element method reduces the size of the system matrices rather than attempting to introduce sparsity. The basic idea is that the conventional polynomial BE shape functions are multiplied with 
plane wave functions. The number of plane waves and their direction may be chosen freely. Evenly distributed directions are most commonly applied, but they may be irregularly distributed if some knowledge about a prevailing wave direction is available. Just like with the partition-of-unity FEM and other methods that apply plane wave functions, the presence of a large number of plane waves causes the conditioning of the system to degrade.

A recently developed class of boundary element methods is the so-called wave number independent methods. These methods try to adapt the conventional BEM so that the prediction accuracy obtained with a given discretization becomes independent of frequency, or at least that the computational costs grow with frequency at a sublinear, namely logarithmic, rate. The idea is to enrich the $\mathrm{BE}$ shape functions by incorporating the known asymptotic behaviour of the solution at high frequencies. The integrands involved with the new BE approaches may be decomposed into a highly oscillating factor and a smooth factor. Depending on the numerical scheme applied to incorporate the boundary conditions, single or double integrals have to be solved. Chandler-Wilde et al. [104] apply a weighted residual formulation and end up with highly oscillatory double integrals. Arden et al. [105] describe a similar method, adapting a collocational scheme and having the advantage that only highly oscillatory single integrals have to be evaluated. In this respect, the work of Huybrechs and Vandewalle [106] is mentioned as a cost efficient method for evaluation of single integrals and Huybrechs and Vandewalle [107] for the efficient evaluation of multidimensional highly oscillatory integrals. Evaluation of both the weighted residual approach and the collocational approach on 2D scattering problems by convex polygons, shows that both methods indeed exhibit only a logarithmic increase in computational cost as frequency increases. Huybrechs and Vandewalle [108] and Huybrechs [109] extend the collocational method by introduction of very effective quadrature rules for the oscillatory integrals and obtain a sparse discretization matrix for the problem. It is found that matrix entries are non-zero only if at least one quadrature point exists that lies in the support of the basis function. Therefore, the number of non-zero points depends on the size of the supports of the basis functions. If all basis functions are local, then the structure of the matrix will be sparse. At the moment, the wave number independent $\mathrm{BE}$ methods are restricted to convex obstacle scattering problems. For non-convex obstacles, the asymptotic behaviour at high frequencies is not available in an applicable form.

\subsubsection{Multipole methods}

The key idea of the fast multipole BEM $[110,111,112,113,114,115,116]$ is to utilize two formulations for the fundamental Green's kernel solution, one in the near-field and one in the far-field. The effect of sources far away from a field point apply a grouped multipole expansion, whereas for nearby sources standard BEM evaluations are used. A multi-level approach is applied in that the discretized boundary surface is fitted to a binary cluster tree. A cluster is a collection of boundary elements which are located within a tolerance range from the cluster's centre. The basic idea is that only neighbouring clusters are considered to be in the considered cluster's near-field. These contributions are computed directly by standard BEM evaluations. The children of the considered cluster's parent cluster are added to a so-called 'interaction' set and appropriate multipole evaluations are performed. This procedure is repeated upwards until the top of the tree is reached, after which the determined contributions are passed downwards until they reach the lowest level. On the lowest level, the results are recovered for each cluster. In this way, the multipole BEM algorithm solves the problem with a computational cost that depends quasi linearly on the number of unknowns, i.e. a complexity of $N \log N$ is obtained instead of $N^{2}$ with conventional BEM.

It is stated by various authors that the developments from the generalized methods may be combined with a multi-level multipole approach, even further optimizing the modelling 
procedure.

\section{TREFFTZ-BASED METHOD}

\subsection{Basic formulations}

\subsubsection{Origin}

The discussion of the enhancements and extensions of both the FEM and the BEM has revealed that the introduction of a priori knowledge of the exact solution improves the computational efficiency of these methods substantially. Already in 1926, Trefftz [17] proposes the use of this a priori knowledge in the definition of approximate solutions. His approximate solutions satisfy the governing domain equations but violate the boundary conditions. Originally, Trefftz has introduced his approach as a counterpart for the Rayleigh-Ritz approach in order to obtain an error bound on the Rayleigh-Ritz prediction for a potential problem. Only half a century later, researchers have picked up the idea of Trefftz again as they recognize its potential advantages in numerical prediction methods. This has led to a class of numerical methods which are generally called Trefftz-based methods. The reader is referred to Jirousek and Wróblewski [18] for a historical overview of the development of the Trefftz-based methods, while Kita and Kamiya [19] provide a detailed overview of most accepted Trefftz-based methods.

In the mid-1990s, the Trefftz-based methods have made their entry in the field of computational acoustics. One of the first attempts to apply this method to the homogeneous Helmholtz equation has been reported by Cheung et al. [117]. The original approach of Trefftz is detailed in that publication for the calculation of wave forces on offshore structures and is reformulated here for acoustics. The purpose of this outline is merely to illustrate the basics of the Trefftz-based method. Subsequent sections provide a more detailed survey of different Trefftz-based methods.

\subsubsection{Indirect formulation}

Cheung et al. [117] consider the Helmholtz problem for an unbounded domain. The Sommerfeld radiation condition in equation (4) or (5) applies in order to model the far-field at infinity $\Gamma_{\infty}$ correctly

$$
\begin{aligned}
& \text { 2D: } \lim _{r \rightarrow \infty} \sqrt{r}\left(\frac{\partial p(\mathbf{r})}{\partial r}+j k p(\mathbf{r})\right)=0, \\
& \text { 3D: } \lim _{r \rightarrow \infty} r \quad\left(\frac{\partial p(\mathbf{r})}{\partial r}+j k p(\mathbf{r})\right)=0 .
\end{aligned}
$$

Neumann boundary conditions have been specified on the remaining boundaries $\Gamma_{v}$. The approximate solution $\hat{p}(k, \mathbf{r})$ is selected from the following set of complete solutions [118]

$$
\begin{aligned}
& \text { 2D: } p(k, \mathbf{r})=a_{0} H_{0}^{(1)}(k r)+\sum_{m=1}^{\infty}\left(a_{m} H_{m}^{(1)}(k r) \cos (m \theta)+b_{m} H_{m}^{(1)}(k r) \sin (m \theta)\right), \\
& \text { 3D: } p(k, \mathbf{r})= \\
& \quad \sum_{m=1}^{\infty} \sum_{q=-m}^{m}\left(a_{m q} h_{m}^{(1)}(k r) P_{m}^{q}(\cos \theta)\right),
\end{aligned}
$$

which are defined in cylindrical coordinates $\mathbf{r}=(r, \theta)$ for the $2 \mathrm{D}$ case and in spherical coordinates $\mathbf{r}=(r, \theta, \psi)$ for the $3 \mathrm{D}$ case. In equation (44), the functions $H_{m}^{(1)}, h_{m}^{(1)}$ and $P_{m}^{q}$ are the Hankel functions of the first kind, the spherical Hankel functions of the first kind and the associated Legendre polynomials, respectively. 
The approximate solution $\hat{p}$ is obtained by truncating the set of complete solutions in equation (44). It can be expressed as follows

$$
\hat{p}(k, \mathbf{r})=\sum_{b=1}^{n_{b}} \Phi_{b}(k, \mathbf{r}) \alpha_{b}=\boldsymbol{\Phi}(k, \mathbf{r}) \boldsymbol{\alpha}, \quad \forall \mathbf{r} \in \Omega,
$$

where $\Phi_{b}(k, \mathbf{r})$ represent the wave functions selected from the complete set of solutions and $\alpha_{b}$ represent the unknown contribution factors. The wave functions $\Phi_{b}$ are collected in the row matrix $\boldsymbol{\Phi}$ and the corresponding contribution factors are stored in the vector $\boldsymbol{\alpha}$. An auxiliary matrix $\boldsymbol{\Psi}$ is defined here, which simplifies the expressions for the Trefftz model matrices and vectors to come

$$
\boldsymbol{\Psi}\left(\mathbf{r}_{b}, k\right)=\frac{j}{\rho \omega} \frac{\partial}{\partial n} \boldsymbol{\Phi}\left(\mathbf{r}_{b}, k\right), \quad \forall \mathbf{r}_{b} \in \partial \Omega .
$$

The approximation $\hat{p}$ satisfies the homogeneous Helmholtz equation and the Sommerfeld radiation condition a priori. Only the violation of the Neumann boundary conditions needs to be enforced to zero in an integral sense by application of the weighted residual approach

$$
\int_{\Gamma_{v}} u\left(\frac{j}{\rho \omega} \frac{\partial \hat{p}}{\partial n}-\bar{v}_{n}\right) \mathrm{d} \Gamma=0 .
$$

A Galerkin approach is followed similar to the FEM, i.e. each basis function $\Phi_{b}$ is selected as weighting function. Together with the substitution of the approximation in equation (47), this results in a set of $n_{b}$ algebraic equations of the following Trefftz boundary integral model

$$
\mathbf{A}(k) \boldsymbol{\alpha}=\mathbf{b}(k)
$$

with the Trefftz system matrix: $\mathbf{A}(k)=\int_{\Gamma_{v}} \mathbf{\Phi}(k, \mathbf{r})^{T} \mathbf{\Psi}(k, \mathbf{r}) \mathrm{d} \Gamma$

and the Trefftz boundary vector: $\mathbf{b}(k)=\int_{\Gamma_{v}} \mathbf{\Phi}(k, \mathbf{r})^{T} \bar{v}_{n} \mathrm{~d} \Gamma$.

The outlined method is according to the original idea of Trefftz. The unknown degrees of freedom in the solution vector $\boldsymbol{\alpha}$ do not represent physical field quantities. Therefore, this version is a member of the family of the indirect Trefftz-based methods.

\subsubsection{Direct formulation}

In addition to the indirect Trefftz-based methods, a direct version of the method exists where the unknown degrees of freedom do represent physical boundary field quantities. Cheung et al. [117] have applied the direct Trefftz-based method for the same unbounded Helmholtz problem. This method is also explained briefly here for acoustics. The main difference with the indirect version is that the approximate solution $\hat{p}$ is not selected from the set of complete solutions. Therefore, this approximation violates the homogeneous Helmholtz equation in the unbounded domain $\Omega$, the Sommerfeld radiation condition at infinity $\Gamma_{\infty}$ and the Neumann boundary condition on $\Gamma_{v}$. The equation of motion (1b) is however satisfied. The following weighted residual formulation enforces the involved errors to zero in an integral sense

$$
\int_{\Omega} u\left(\Delta \hat{p}+k^{2} \hat{p}\right) \mathrm{d} \Omega+\int_{\Gamma_{\infty}} j \rho \omega u\left(\frac{j}{\rho \omega} \frac{\partial \hat{p}}{\partial n}-\frac{\hat{p}}{\rho c}\right) \mathrm{d} \Gamma+\int_{\Gamma_{v}} j \rho \omega u\left(\frac{j}{\rho \omega} \frac{\partial \hat{p}}{\partial n}-\bar{v}_{n}\right) \mathrm{d} \Gamma=0 .
$$


This relation can be rewritten as follows using the Green's second identity formula

$$
\int_{\Omega} \hat{p}\left(\Delta u+k^{2} u\right) \mathrm{d} \Omega+\int_{\Gamma_{\infty}} j \rho \omega \hat{p}\left(\frac{j}{\rho \omega} \frac{\partial u}{\partial n}-\frac{u}{\rho c}\right) \mathrm{d} \Gamma-\int_{\Gamma_{v}}\left(\hat{p} \frac{\partial u}{\partial n}+j \rho \omega u \bar{v}_{n}\right) \mathrm{d} \Gamma=0 .
$$

The first two terms in this relation vanish for the following choice of the weighting function

$$
u(k, \mathbf{r})=\sum_{b=1}^{n_{b}} \Phi_{b}(k, \mathbf{r}) \beta_{b}=\boldsymbol{\Phi}(k, \mathbf{r}) \boldsymbol{\beta}, \quad \forall \mathbf{r} \in \Omega,
$$

where $\Phi_{b}(k, \mathbf{r})$ represent wave functions selected from the complete set of solutions in equation (44) and $\beta_{b}$ represent arbitrary contributions stored in the vector $\boldsymbol{\beta}$. Note that the approximate solution $\hat{p}=\hat{p}(k, \mathbf{r})$ needs only to be defined on the boundary $\Gamma_{v}$ similar to the direct BEM for which the following $\mathrm{FE}$ approximation is well suited

$$
\hat{p}(\mathbf{r})=\hat{p}\left(\mathbf{r}_{b}\right)=\mathbf{N}\left(\mathbf{r}_{b}\right) \mathbf{p}, \quad \forall \mathbf{r}_{b} \in \Gamma_{v}
$$

The substitution of $u$ and $\hat{p}$ in equation (50) and the requirement that the resulting relation should hold for all possible contribution vectors $\boldsymbol{\beta}$ results in the direct Trefftz boundary integral model

$$
\mathbf{A}(k) \mathbf{p}=\mathbf{b}(k)
$$

with the direct Trefftz system matrix: $\mathbf{A}(k)=\int_{\Gamma_{v}} \mathbf{\Psi}(k, \mathbf{r})^{T} \mathbf{N}\left(\mathbf{r}_{b}\right) \mathrm{d} \Gamma$

and the direct Trefftz boundary vector: $\mathbf{b}(k)=\int_{\Gamma_{v}} \mathbf{\Phi}(k, \mathbf{r})^{T} \bar{v}_{n} \mathrm{~d} \Gamma$.

This boundary integral model is solved for the unknown degrees of freedom in the solution vector $\mathbf{p}$, which, contrary to the indirect version, represent physically meaningful quantities, namely nodal boundary pressure values. Notice that the direct Trefftz model in equation (53) is very similar to the direct boundary element model. The discriminating feature is the use of a set of complete functions instead of the use of fundamental solutions as kernel functions in the BEM.

The direct Trefftz-based method is not as popular as the indirect version for acoustic applications. Apart from Cheung et al. [117], Masson et al. [119] and Sladek et al. [120], further use of the direct method in acoustics is limited. Therefore, the remainder of this review paper considers only indirect methods. These are referred to as the Trefftz-based methods from here onward.

To conclude this outline of the basics of the Trefftz-based method, the duality of this method is emphasized [121]. On the one hand, the Trefftz-based method is a boundary integral method similar to the BEM, however with the advantage that it does not require the evaluation of singular integrals. On the other hand, it is based on a similar weighted residual formulation with trial and test functions as the FEM.

\subsection{Survey of Trefftz-based methods}

\subsubsection{Classification}

The classification of the Trefftz-based methods for time-harmonic acoustics is not a straightforward task. The reason for this originates mainly from its duality. Researchers with different backgrounds have embraced the method and have focused on a specific feature. Researchers with a FEM background consider more often interior acoustic problems or 
near-field acoustic radiation problems. The boundary of the acoustic domain can have a rather complex shape which causes numerical difficulties. The number $n_{b}$ of Trefftz basis functions $\Phi_{b}$ in equation (45) must increase for accuracy reasons but at the same time the model becomes ill-conditioned [122]. The numerical condition is kept under control by subdividing the problem domain in smaller simply shaped subdomains similar to the FEM. This subdivision principle characterizes the development of Trefftz-elements [18, 19, 123, 124].

Researchers with a BEM background, on the other hand, are often interested in exterior acoustic problems such as scattering or radiation problems. They are more familiar with the use of fundamental free-field solutions and potential layers as kernel functions. These functions can be considered as continuously distributed acoustic sources along the finite part of the problem boundary $\Gamma_{f}=\Gamma_{p} \cup \Gamma_{v} \cup \Gamma_{Z}$. Based on these ideas, the numerical condition of the Trefftz-based model can also be kept under control by combining different sets of complete functions rather than by following a domain subdivision procedure. The different sets of complete functions correspond to multiple acoustic sources in this case, which are distributed near the scatterer or radiator. The use of multiple source solutions characterizes the class of source simulation methods [125].

The Trefftz-based methods can also be classified by the way the boundary conditions are enforced. Three well-known strategies are used based on either (i) a collocation scheme, (ii) a least-squares formulation or (iii) a Galerkin approach. Furthermore, two large classes of Trefftz-elements exist based on the treatment of the continuity conditions between elements, namely the hybrid Trefftz-elements and the frameless Trefftz-elements [18]. The wave based method, which is detailed further on, belongs to the class of the frameless Trefftz-elements. Actually, a third approach is available to enforce the continuity conditions along subdomain interfaces, namely the ultra-weak variational formulation [126]. This technique is unique in its kind and it cannot be categorized by the other two classes of Trefftz-elements.

Finally, some meshless methods have been developed which are strongly related to the indirect Trefftz-based method. They are not based on the moving least-squares method [86] such as the element-free Galerkin method (see section 3.4.4) but they are based on radial basis functions [127].

Based on the above discussion, the Trefftz-based methods are arranged in five categories, which are

1. the hybrid Trefftz finite element methods,

2. the frameless Trefftz finite element methods,

3. the ultra-weak variational formulation,

4. the source simulation methods and

5. the radial basis function methods.

The remaining part of this chapter reviews the stat-of-the-art in these categories.

\subsubsection{Hybrid Trefftz finite element method}

The hybrid Trefftz FEM is the first method in the class of the Trefftz-element methods. It incorporates the indirect Trefftz-based method in the FEM as the name already suggests. The characteristic feature of the hybrid Trefftz FEM is that the inter-element continuity is enforced by application of the Langrange multiplier technique. The followed FE strategy is summarized below for an interior acoustic problem with Neumann boundary conditions [128].

1. The acoustic domain $\Omega_{i}$ is subdivided in elements $\left(\Omega_{i}=\cup_{e=1}^{n_{e}} \Omega^{e}\right)$ and an auxiliary frame is placed along inter-element interfaces $\Gamma_{i}^{e}$ (see figure 9 ). 
2. Within each element $\Omega^{e}$, a truncated set of complete functions approximates the exact solution and a simple FE interpolation approximates the unknown frame variable $\lambda$

$$
\begin{aligned}
& p(\mathbf{r}, k) \approx \hat{p}(\mathbf{r}, k)=\mathbf{\Phi}(\mathbf{r}, k) \boldsymbol{\alpha}^{e}, \forall \mathbf{r} \in \Omega^{e} \\
& \lambda\left(\mathbf{r}_{b}\right) \approx \hat{\lambda}\left(\mathbf{r}_{b}\right)=\mathbf{N}\left(\mathbf{r}_{b}\right) \boldsymbol{\lambda}^{e}, \quad \forall \mathbf{r}_{b} \in \Gamma_{i}^{e}
\end{aligned}
$$

where the vectors $\boldsymbol{\alpha}^{e}$ and $\boldsymbol{\lambda}^{e}$ contain the Trefftz and frame degrees of freedom.

3. The following weighted residual formulation enforces the errors on the boundary conditions and the continuity conditions to zero

$$
\begin{array}{r}
\int_{\Gamma_{v}^{e}} u\left(\frac{j}{\rho \omega} \frac{\partial \hat{p}}{\partial n}-\bar{v}_{n}\right) \mathrm{d} \Gamma+\int_{\Gamma_{i}^{e}} u\left(\frac{j}{\rho \omega} \frac{\partial \hat{p}}{\partial n}-\hat{\lambda}\right) \mathrm{d} \Gamma=0, \\
-\int_{\Gamma_{i}^{e}} \mu\left(\hat{p}-p_{i}\right) \mathrm{d} \Gamma=0,
\end{array}
$$

where $u$ and $\mu$ represent arbitrary test functions and $p_{i}$ is the pressure on the frame. The frame pressure $p_{i}$ is not considered further since its contribution vanishes during the assembly (conforming Trefftz-element).

4. The application of the Galerkin approach together with the substitution of the pressure and frame approximation results in the hybrid Trefftz-element

$$
\left[\begin{array}{cc}
\mathbf{A} & \mathbf{C}^{T} \\
\mathbf{C} & \mathbf{0}
\end{array}\right]\left\{\begin{array}{l}
\boldsymbol{\alpha}^{e} \\
\boldsymbol{\lambda}^{e}
\end{array}\right\}=\left\{\begin{array}{l}
\mathbf{b} \\
\mathbf{0}
\end{array}\right\} \text { with } \mathbf{C}(k)=-\int_{\Gamma_{i}^{e}} \mathbf{N}\left(\mathbf{r}_{b}\right)^{T} \boldsymbol{\Phi}(\mathbf{r}, k) \mathrm{d} \Gamma
$$

and with $\mathbf{A}$ and $\mathbf{b}$ similar to the definition in equation (48).

5. The assembly of all elements results in the hybrid Trefftz FE model.

The hybrid Trefftz FEM outlined here is a so-called hybrid Trefftz traction FEM [18]. The term 'traction' refers to the equivalent mechanical quantity for the normal velocity, which is selected as Lagrange multiplier. Several methods with other Lagrange multipliers have been developed for other engineering disciplines, but this choice is one of few reported in literature $[129,130]$. Farhat et al. [129] derive this method, which is named the discontinuous Galerkin method, from the discontinuous enrichment method (see section 3.4.5) by omitting the coarse scale FE approximation $\hat{p}$ from the solution.

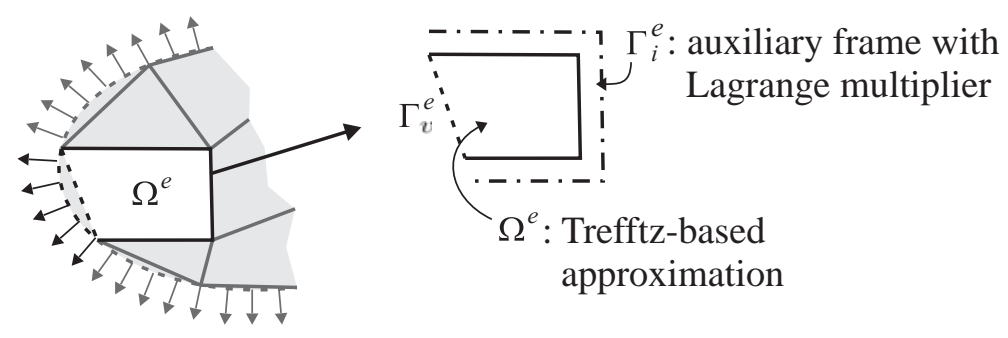

Figure 9: Hybrid Trefftz finite element 


\subsubsection{Frameless Trefftz finite element method}

Frameless Trefftz-elements impose the inter-element continuity conditions without the use of an auxiliary frame. A general FE framework is followed which is again summarized for an interior acoustic problem with Neumann boundary conditions.

1. The acoustic domain $\Omega_{i}$ is subdivided in elements $\left(\Omega_{i}=\cup_{e=1}^{n_{e}} \Omega^{e}\right)$.

2. The Trefftz-based approximation in equation (54a) is used $\left(\hat{p}(\mathbf{r}, k)=\mathbf{\Phi}(\mathbf{r}, k) \boldsymbol{\alpha}^{e}\right)$.

3. The application of a method dependent integral formulation enforces the errors on the boundary conditions and the continuity conditions to zero in an average way. This is detailed below for three frameless Trefftz-elements.

4. The substitution of the Trefftz-based approximation and, if necessary, of the test functions results in the general expression for the frameless Trefftz-element

$$
\mathbf{A}^{e} \boldsymbol{\alpha}^{e}+\mathbf{C}^{e g} \boldsymbol{\alpha}^{g}=\mathbf{b}^{e} .
$$

The model matrices and vector are method dependent. They are detailed below.

5. The assembly of all elements results in the frameless Trefftz FE model.

The first method of this type is the Trefftz least-squares FEM introduced by Jirousek and Wróblewski [131] for a static mechanical problem and it has been applied successfully to the Helmholtz problem [132, 133, 134]. The method uses a least-squares formulation to impose the boundary conditions and the continuity conditions. The integral formulation in step 3 above results from the minimization of an error functional $\mathcal{F}$ with the following general format

$$
\mathcal{F}=\int_{\Gamma_{v}^{e}} \beta_{v}\left|\frac{j}{\rho \omega} \frac{\partial \hat{p}^{e}}{\partial n}-\bar{v}_{n}\right|^{2} \mathrm{~d} \Gamma+\int_{\Gamma_{i}^{e}}\left(\beta_{p}\left|\hat{p}^{e}-\hat{p}^{g}\right|^{2}+\beta_{v}\left|\frac{j}{\rho \omega} \frac{\partial \hat{p}^{e}}{\partial n}-\frac{j}{\rho \omega} \frac{\partial \hat{p}^{g}}{\partial n}\right|^{2}\right) \mathrm{d} \Gamma
$$

where the parameters $\beta_{p}$ and $\beta_{v}$ restore the the homogeneity of the physical dimensions. The general expressions for the model matrices in equation (57) are given by

$$
\begin{aligned}
\mathbf{A}^{e} & =\int_{\Gamma_{i}^{e}} \beta_{p}\left(\boldsymbol{\Phi}^{e}\right)^{H} \boldsymbol{\Phi}^{e} \mathrm{~d} \Gamma+\int_{\Gamma^{e}} \beta_{v}\left(\mathbf{\Psi}^{e}\right)^{H} \mathbf{\Psi}^{e} \mathrm{~d} \Gamma \\
\mathbf{b}^{e} & =\int_{\Gamma_{v}^{e}} \beta_{v}\left(\boldsymbol{\Psi}^{e}\right)^{H} \bar{v}_{n} \mathrm{~d} \Gamma \\
\mathbf{C}^{e g} & =\int_{\Gamma_{i}^{e}}\left(-\beta_{p}\left(\boldsymbol{\Phi}^{e}\right)^{H} \boldsymbol{\Phi}^{g}+\beta_{v}\left(\boldsymbol{\Psi}^{e}\right)^{H} \mathbf{\Psi}^{g}\right) \mathrm{d} \Gamma
\end{aligned}
$$

where $\bullet^{H}$ denotes the conjugate transpose operator. Notice that the auxiliary matrix $\Psi^{g}$ is defined for the outward normal vector $\mathbf{n}$ of the adjacent element $\Omega^{e}$.

A second frameless Trefftz method is the variational theory of complex rays [135], which is a dedicated numerical method for the prediction of mid-frequency vibrations which is able to yield numerical predictions with the same level of accuracy in the mid-frequency range as the FEM, however with substantially less computational efforts. The considered problem structure must lend itself to partitioning into homogeneous substructures. The first characteristic of the approach is the use of a variational formulation of the problem which 
enables application of a priori independent approximations within each substructure. In other words, it is not necessary for the approximations to satisfy a priori the compatibility and the equilibrium conditions at the interfaces between substructures. Instead, these conditions are incorporated into the variational formulation. The second characteristic of the variational theory of complex rays is the introduction within each substructure of twoscale approximations, having a strong mechanical meaning. The solution is assumed to be well-described as the superposition of an infinite number of local vibration modes. These basic modes (which can be interior modes, boundary modes or corner modes and which represent wave solutions of various forms) satisfy the equations of motion. In this way, the method is a Trefftz method. The modes constitute two-scale approximations with a slowly varying scale and a rapidly varying scale. Only the slowly varying scale, i.e. the polynomial part of the solution, is discretized, while the rapidly varying scale, i.e. the oscillating part of the solution, is taken into account analytically. The unknowns are the discretized amplitudes of the slowly varying parts. As a result, no fine discretization is needed and the approximate solution is obtained from a small, computationally efficient numerical model, as compared to the large conventional FE models.

A third frameless Trefftz method is the Wave Based Method (WBM) [136]. The WBM is similar to the Trefftz least-squares FEM in that it also applies a direct coupling of nonconforming fields used to describe the dynamic response variables within (large) Trefftz elements. Two essential differences distinct both methods. First, there is the selection of the applied functions. In the Trefftz least-squares FEM, plane wave functions [133] or Bessel functions [134] are applied. In the WBM a combination of propagating and evanescent waves is applied. A second difference is the treatment of the boundary and continuity conditions. The WBM adopts a weighted residual approach to enforce the boundary and continuity residuals to zero. Unlike the Trefftz least-squares FEM, where both pressure and (normal) velocity continuity are enforced for all subdomains associated with an interface, the WBM only enforces one relation for each subdomain adjacent to the interface. The WBM has been successfully applied for many time-harmonic interior acoustic problems [137], interior vibro-acoustic problems [20] and exterior vibro-acoustic problems [23]. It is shown that the WBM exhibits an enhanced computational efficiency as compared to conventional FEMs. As a result the WBM is able to tackle problems at higher frequencies than the FEM. In order to fully benefit from the WBM's computational efficiency, the considered subdomains should have a relatively moderate geometrical complexity. This has triggered investigations for a hybrid finite element-wave based method $[128,138]$ in order to tackle problems with more complex geometrical descriptions. In coupling the FE domains with the WB domains, two strategies can be followed. Either a direct coupling approach is applied, like is used in the WBM itself, or an indirect coupling approach is used, requiring the introduction of auxiliary frames like with the hybrid Trefftz methods. The hybrid finite element-wave based method has proven to be successful for several interior acoustic problems [139] and interior vibro-acoustic problems with an acoustic-acoustic coupling [24] and an acoustic-structural coupling [140].

Sections 6 and 7 will elaborate on the basic formulations of the WBM for 2D timeharmonic acoustic problems and will illustrate the method's enhanced computational performance, as compared to conventional element based methods, for both interior and exterior acoustic problems.

\subsubsection{Ultra-weak variational formulation}

The ultra weak variational formulation [126] is based on a discretization of the problem domain into elements. A boundary variable is introduced at the element boundaries satisfying an alternative variational formulation, namely the ultra weak variational formulation. It is shown that, if the boundary variable satisfies the ultra weak variational formulation, 
the associated field variable, representing the dynamic pressure, satisfies the Helmholtz equation. The field variable is approximated by a set of plane wave functions. Application of the Galerkin approach yields the ultra weak variational formulation matrix equation. Interelement continuity is taken directly into account in the weak variational formulation. In this way the ultra weak variational formulation is comparable to the Trefftz least-squares FEM. Similar to the finite element tearing and interconnecting approach, the ultra weak variational formulation applies alternative coupling conditions based on equivalent normal velocities in order to overcome the ill-posedness of the conventional pressure and normal velocity continuity [141]. Once the boundary variable is identified from solution of the system of equations, the associated field variable may be recovered. Preconditioning of the system of equations may be useful when large-scale problems are solved using iterative methods. Although the ultra weak variational formulation promises to solve wave problems at reduced computational cost, it may suffer from numerical instability.

\subsubsection{Source simulation method}

Regardless of the various names given to this class of methods (e.g. superposition method, source simulation technique, equivalent source method, full-field method, null-field method, equivalent sphere method, energy source simulation method, etcetera) they all apply the same modelling principle. An interior/exterior acoustic boundary value problem is solved by positioning a number of sources exterior/interior to the problem boundary (see figure 10). Enforcement of the boundary conditions yields a numerical model, which can be solved to obtain the source strengths of the applied sources. As all Trefftz methods, these methods yield ill-conditioned matrices.

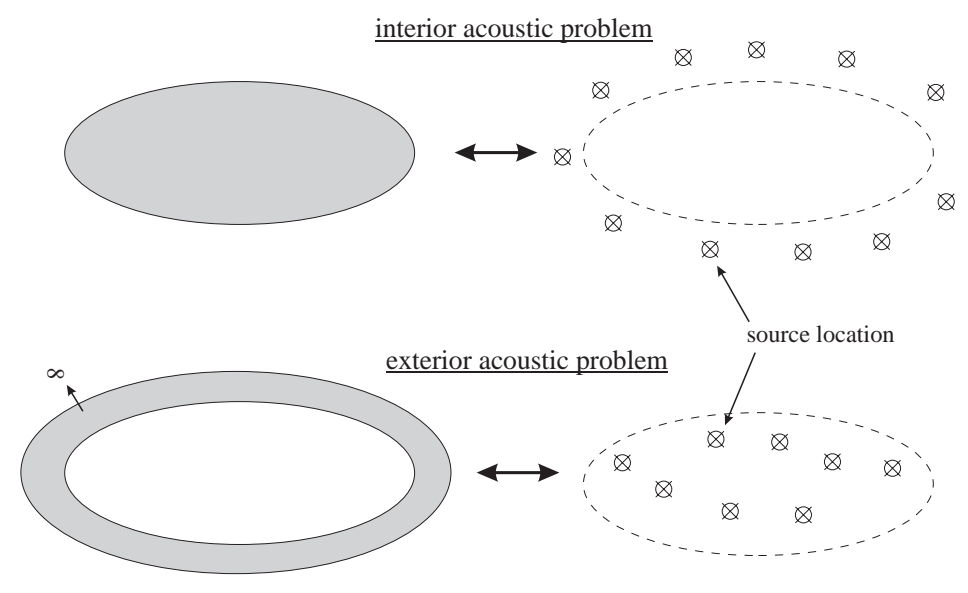

Figure 10: Application of the equivalent source method for interior and exterior problems

Koopmann et al. [142] discuss the basic approach of putting a number of sources inside a radiating boundary and enforcing the boundary conditions at the boundary via a collocational scheme to determine their source strengths. Enforcing the boundary conditions via a Galerkin weighted residual formulation yields the so-called null-field equation method [143], enforcement via a least-square formulations yields the L2 method [144]. Ochmann [125] gives a comprehensive overview of the different ways to incorporate the boundary conditions. Inside the radiating boundary, either few source locations are chosen, but at every source location a large number of sources with increasing order is used, or a lot of simple (monopole) sources are employed at various locations. The latter is often denoted as the superposition method or the equivalent source method. However, no general rules exists for the determination of the number of sources, their locations and their orders, which turns out 
to be very problem dependent. Ochmann [125] mentions that for the one-point multipole method the convergence to the true solution can be shown if the surface is assumed to be a Lyapunov surface, which has the essential property that the normal vector to the surface is uniquely defined at each point, i.e. a smooth surface. Furthermore, numerical instabilities can occur with some variants of the source simulation technique. Ochmann [145] develops a new stabilized variant of the source simulation technique, called the full-field method, by using the exterior instead of the interior Helmholtz integral equation. When unconjugated weighting functions are applied, the method is denoted the full-field method of the first kind, while the full-field method of the second kind applies complex conjugate weighting functions. The method is more stable since the resulting matrix becomes more diagonally dominant. Recently, Ochmann [146] applied the equivalent source method for the sound propagation over an infinite impedance plane.

In order to determine rules for positioning the sources, Pavic [147] determined a greedy algorithm for optimal selection of the monopole source positions for $2 \mathrm{D}$ problems. The strategy does not yield a strict mathematical optimum, but gives nonetheless very satisfactory results when compared to random or uniform positioning. Pavic [148] optimizes the approach so that also multipole sources can be incorporated. Simulations are limited to 2D problems. Application for 3D problems may turn out to be too time consuming.

Bouchet et al. [149] propose a method which uses an equivalent sphere instead of a multipole point source. The number of unknowns is equal to the number of radiation modes of the sphere. The unknown radiation mode contribution factors are obtained from a quadratic residual minimization procedure. This approach has two advantageous features. The first is that the modes of the spheres may be normalized, having a stabilizing effect. Secondly, if each point of the boundary structure corresponds to a point of the sphere, the vibrating field at the surface of the sphere may be deduced from that of the boundary structure by geometric mapping. For simple 3D problems, a significant reduction in calculation time compared to the BEM is obtained.

Reboul et al. [150] apply an equivalent source method, using acoustical energy sources instead of pressure sources. Validation calculations on the radiation of a ribbed plate yield favourable results. Herrin et al. [151] developed a similar technique to determine the sound energy density called the energy source simulation method. A specified intensity boundary condition on the surface of a vibrating body is approximated by a combination of energy density sources located outside the acoustic domain by application of a least-square minimization procedure. In most cases, the method gives reliable results.

\subsubsection{Radial basis function methods}

The boundary knot method [152] is a method that uses Bessel functions to solve the Helmholtz equation. However, the method stems from the methods based on the so-called Radial Basis Functions (RBFs) [127]. Kansa's method, also called the radial basis functions collocation method, applies globally defined RBF interpolation functions and a collocational formulation to obtain the solution of partial differential equations. These RBFs may be considered as source terms and are distributed inside the domain. The RBFs are no exact solutions of the differential equations and therefore, collocation points are not only required at the boundary, but also inside the domain, which distinguishes RBF based methods from true Trefftz methods. Kansa's method yields very ill-conditioned matrices and, in a way, its modelling approach is comparable to that from the element free Galerkin method. The method of fundamental solutions is a modified form of Kansa's method, in that it applies singular functions that exactly satisfy the governing differential equation. The method of fundamental solutions is comparable to the Trefftz equivalent source methods since also sources are positioned outside the problem domain while the source strengths are determined by enforcing the boundary conditions at the boundary in a collocational 
way $[153,154]$. No collocation points are needed inside the domain. However, the method of fundamental solutions does not apply T-complete functions, distinguishing it from the genuine Trefftz methods. Also a weighted residual [155] or a least-squares [156] approach may be applied to incorporate the boundary conditions. In the dual reciprocity method of fundamental solutions [157] the solution field is cast into a homogeneous and a particular solution. First, the particular solution is solved regardless of the boundary conditions using global interpolation of the field in terms of RBFs. When the particular solution is known, the homogenous solution can be tackled with the method of fundamental solutions. The boundary knot method is a dual reciprocity method of fundamental solutions based on a collocational scheme, yielding severely ill-conditioned matrices. A major distinctive feature of the boundary knot method is that it applies non-singular functions to approximate the homogeneous solution. As a result the sources may be located on the boundary and no artificial surface collecting the sources outside the problem domain is required. Since not all applied approximation functions are exact solutions of the governing differential equation, i.e. the RBFs applied for the approximation of the particular solution, also collocation points inside the domain are required. The applied fundamental solutions are Bessel functions. However, they do not form a T-complete set of functions.

\section{WAVE BASED METHOD}

\subsection{Basic formulations}

The wave based method (WBM) is a frameless Trefftz-based prediction technique (see section 5.2.3) which is applicable for time-harmonic acoustic problems. As a result, the method is based on an indirect Trefftz approach and follows a mathematical formulations related to the one discussed in section 5.1.2. In general, five major steps are distinguished in a WB modelling procedure:

1. partitioning into a number of convex subdomains,

2. selection of a sets of wave functions,

3. construction of the WB system matrices,

4. solution of the wave model,

5. and postprocessing.

\subsubsection{Partitioning into convex subdomains}

A sufficient condition for the WB approximations to converge towards the exact solution, is convexity of the considered problem domain [136]. In a general acoustic problem, see figures 1 and 2 , the acoustic problem domain $\Omega$ may be non-convex so that a partitioning into a number of convex subdomains is required. If the WBM is applied for unbounded problems, an initial partitioning of the unbounded domain into a bounded and an unbounded region precedes the partitioning into convex subdomains. Figure 11 illustrates the principle. The unbounded acoustic problem domain $\Omega$ is divided into two non-overlapping regions by a truncation curve $\Gamma_{t}$. The bounded region, enclosed by the truncation curve and the problem boundary $\Gamma_{p} \cup \Gamma_{v} \cup \Gamma_{Z}$ is partitioned into a number of $N_{\Omega}$ non-overlapping, convex subdomains $\Omega^{(\alpha)}$. The unbounded region exterior to $\Gamma_{t}$ is considered as one acoustic subdomain $\Omega^{\left(N_{\Omega}+1\right)}$. As a result, the acoustic problem domain is partitioned into $N_{\Omega}+1$ subdomains: $\Omega=\bigcup_{\alpha=1}^{N_{\Omega}+1} \Omega^{(\alpha)}$.

Since the bulk part of the computational efforts is spent in the creation of the WB system matrices, and since these efforts are linked to the total interface length, it can be 
seen from figure 11 that the efficiency of the WBM is primarily exploited for problems of moderate geometrical complexity. For problems with a more complex boundary description, a hybrid finite element-wave based approach is more suited [128, 138].

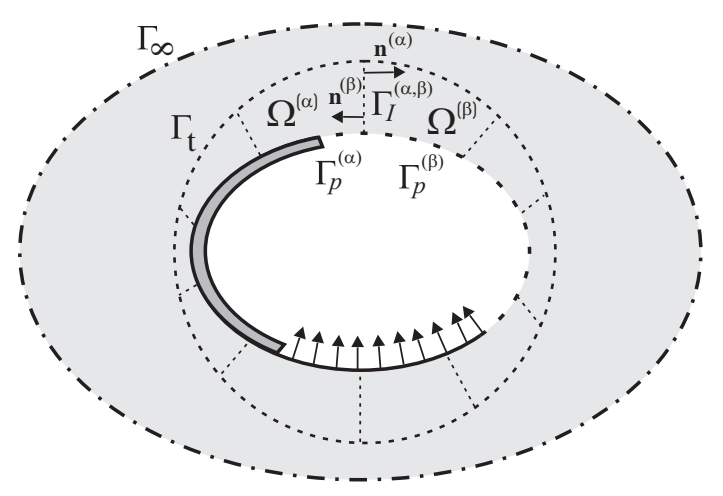

Figure 11: WB partitioning of an exterior acoustic problem

The boundary of a bounded acoustic subdomain $\Omega^{(\alpha)}$ is denoted as $\partial \Omega^{(\alpha)}$. It consists of four mutually exclusive parts,

$$
\partial \Omega^{(\alpha)}=\Gamma_{p}^{(\alpha)} \cup \Gamma_{v}^{(\alpha)} \cup \Gamma_{Z}^{(\alpha)} \cup \Gamma_{I}^{(\alpha)},
$$

on which the following conditions apply:

- $\Gamma_{p}^{(\alpha)}=\Gamma_{p} \cap \partial \Omega^{(\alpha)}$ indicates the part of the boundary of subdomain $\Omega^{(\alpha)}$ on which pressure boundary conditions (3a) are prescribed.

- $\Gamma_{v}^{(\alpha)}=\Gamma_{v} \cap \partial \Omega^{(\alpha)}$ indicates the part of the boundary of subdomain $\Omega^{(\alpha)}$ on which normal velocity boundary conditions (3b) are prescribed.

- $\Gamma_{Z}^{(\alpha)}=\Gamma_{Z} \cap \partial \Omega^{(\alpha)}$ indicates the part of the boundary of subdomain $\Omega^{(\alpha)}$ on which normal impedance boundary conditions (3c) are prescribed.

- $\Gamma_{I}^{(\alpha, \beta)}=\Gamma_{I}^{(\beta, \alpha)}=\partial \Omega^{(\alpha)} \cap \partial \Omega^{(\beta)}$ indicates the common interface between subdomain $\Omega^{(\alpha)}$ and subdomain $\Omega^{(\beta)}$. The continuity conditions which need to be applied on this interface are discussed in the following paragraphs. The collection of all subdomain interfaces in which subdomain $\Omega^{(\alpha)}$ is involved is denoted as $\Gamma_{I}^{(\alpha)}=\bigcup_{\beta=1, \beta \neq \alpha}^{N_{\Omega}+1} \Gamma_{I}^{(\alpha, \beta)}$

The boundary of the unbounded acoustic subdomain $\Omega^{\left(N_{\Omega}+1\right)}$ consists of the collection of acoustic interfaces $\Gamma_{I}^{\left(N_{\Omega}+1\right)}$, coinciding with the truncation curve $\Gamma_{t}$, and the boundary at infinity $\Gamma_{\infty}$.

To ensure continuity over the acoustic interfaces $\Gamma_{I}^{(\alpha, \beta)}$ between the acoustic subdomains $\Omega^{(\alpha)}$ and $\Omega^{(\beta)}$, continuity conditions must be applied. Two types of continuity conditions are considered in the WB methodology.

\section{Pressure and velocity continuity}

Both pressure and normal velocity continuity are imposed at the interfaces:

$$
\begin{gathered}
\text { pressure continuity: } \mathbf{r} \in \Gamma_{I}^{(\alpha, \beta)}: p^{(\alpha)}(\mathbf{r})=p^{(\beta)}(\mathbf{r}), \\
\text { normal velocity continuity: } \mathbf{r} \in \Gamma_{I}^{(\alpha, \beta)}: \mathcal{L}_{v}^{(\alpha)}\left(p^{(\alpha)}(\mathbf{r})\right)=-\mathcal{L}_{v}^{(\beta)}\left(p^{(\beta)}(\mathbf{r})\right),
\end{gathered}
$$


with $\mathcal{L}_{v}^{(\alpha)}$ the velocity operator applied in subdomain $\Omega^{(\alpha)}$

$$
\mathcal{L}_{v}^{(\alpha)}=\frac{j}{\rho \omega} \frac{\partial}{\partial n^{(\alpha)}}
$$

The WBM adopts a direct coupling approach in order to couple the two subdomains. This implies that the continuity conditions (61a) and (61b) are directly enforced on the acoustic quantities of the considered subdomains, without introduction of auxiliary interface variables. In order for the problem to be well-posed, one continuity condition is imposed on each subdomain. This means that, on the interface $\Gamma_{I}^{(\alpha, \beta)}$ between the acoustic subdomains $\Omega^{(\alpha)}$ and $\Omega^{(\beta)}$, the pressure $p^{(\beta)}(\mathbf{r})$ is enforced as a pressure boundary condition on subdomain $\Omega^{(\alpha)}$ along $\Gamma_{I p}^{(\alpha, \beta)}=\Gamma_{I}^{(\alpha, \beta)}$ and the normal velocity $-\mathcal{L}_{v}^{(\alpha)}\left(p^{(\alpha)}(\mathbf{r})\right)$ is applied as a normal velocity boundary condition on subdomain $\Omega^{(\beta)}$ along $\Gamma_{I v}^{(\beta, \alpha)}=\Gamma_{I}^{(\beta, \alpha)}$, or vice versa. As a result

$$
\text { if } \Gamma_{I p}^{(\alpha, \beta)} \neq \emptyset \quad \text { then }\left\{\begin{array} { l } 
{ \Gamma _ { I p } ^ { ( \beta , \alpha ) } = \emptyset } \\
{ \Gamma _ { I v } ^ { ( \alpha , \beta ) } = \emptyset } \\
{ \Gamma _ { I v } ^ { ( \beta , \alpha ) } \neq \emptyset }
\end{array} \quad \text { or } \quad \text { if } \quad \Gamma _ { I v } ^ { ( \alpha , \beta ) } \neq \emptyset \quad \text { then } \quad \left\{\begin{array}{l}
\Gamma_{I v}^{(\beta, \alpha)}=\emptyset \\
\Gamma_{I p}^{(\alpha, \beta)}=\emptyset \\
\Gamma_{I p}^{(\beta, \alpha)} \neq \emptyset
\end{array}\right.\right.
$$

with $\emptyset$ the empty set. Furthermore the following notations are introduced

$$
\Gamma_{I p}^{(\alpha)}=\bigcup_{\beta=1, \beta \neq \alpha}^{N_{\Omega+1}} \Omega_{I p}^{(\alpha, \beta)} \text { and } \Gamma_{I v}^{(\alpha)}=\bigcup_{\beta=1, \beta \neq \alpha}^{N_{\Omega+1}} \Omega_{I v}^{(\alpha, \beta)}
$$

\section{Equivalent normal velocity continuity}

By taking a linear combination of the two conditions (61) and by the introduction of a pressure scaling factor $\bar{Z}_{i n t}$, two new continuity conditions can be formulated:

$$
\begin{aligned}
& \mathbf{r} \in \Gamma_{I}^{(\alpha, \beta)}: \mathcal{L}_{v}^{(\alpha)}\left(p^{(\alpha)}(\mathbf{r})\right)-\frac{1}{\bar{Z}_{i n t}} p^{(\alpha)}(\mathbf{r})=-\mathcal{L}_{v}^{(\beta)}\left(p^{(\beta)}(\mathbf{r})\right)-\frac{1}{\bar{Z}_{i n t}} p^{(\beta)}(\mathbf{r}), \\
& \mathbf{r} \in \Gamma_{I}^{(\alpha, \beta)}: \mathcal{L}_{v}^{(\beta)}\left(p^{(\beta)}(\mathbf{r})\right)-\frac{1}{\bar{Z}_{i n t}} p^{(\beta)}(\mathbf{r})=-\mathcal{L}_{v}^{(\alpha)}\left(p^{(\alpha)}(\mathbf{r})\right)-\frac{1}{\bar{Z}_{\text {int }}} p^{(\alpha)}(\mathbf{r}) .
\end{aligned}
$$

The impedance coupling factor $\bar{Z}_{\text {int }}$ is actually a relative weighting factor. For a large value, the normal velocity component predominates the continuity conditions (63), while for a small value the pressure component predominates. This kind of coupling is referred to as impedance or equivalent normal velocity coupling with $\bar{Z}_{i n t}$ being the impedance coupling value. The term equivalent normal velocity results from the fact that relations (63) actually express continuity of an equivalent acoustic velocity quantity. The equivalent velocity operators are defined as

$$
\begin{aligned}
\mathcal{L}_{e q+}^{(\alpha)} & =\frac{j}{\rho \omega} \frac{\partial}{\partial n^{(\alpha)}}-\frac{1}{\bar{Z}_{i n t}}, \\
\mathcal{L}_{e q-}^{(\alpha)} & =-\frac{j}{\rho \omega} \frac{\partial}{\partial n^{(\alpha)}}-\frac{1}{\bar{Z}_{i n t}} .
\end{aligned}
$$


With the operators (64), equations (63) can be reformulated as

$$
\begin{aligned}
& \mathbf{r} \in \Gamma_{I}^{(\alpha, \beta)}: \mathcal{L}_{e q+}^{(\alpha)}\left(p^{(\alpha)}(\mathbf{r})\right)=\mathcal{L}_{e q-}^{(\beta)}\left(p^{(\beta)}(\mathbf{r})\right), \\
& \mathbf{r} \in \Gamma_{I}^{(\alpha, \beta)}: \mathcal{L}_{\text {eq+ }}^{(\beta)}\left(p^{(\beta)}(\mathbf{r})\right)=\mathcal{L}_{\text {eq- }}^{(\alpha)}\left(p^{(\alpha)}(\mathbf{r})\right) .
\end{aligned}
$$

In order for the partitioned problem to be well-posed, condition (65a) is enforced as a normal velocity boundary condition on subdomain $\Omega^{(\alpha)}$ along $\Gamma_{I z}^{(\alpha, \beta)}=\Gamma_{I}^{(\alpha, \beta)}$ and the associated condition $(65 \mathrm{~b})$ is enforced as a normal velocity boundary condition on subdomain $\Omega^{(\beta)}$ along $\Gamma_{I z}^{(\beta, \alpha)}=\Gamma_{I}^{(\beta, \alpha)}$.

The following notation is introduced

$$
\Gamma_{I z}^{(\alpha)}=\bigcup_{\beta=1, \beta \neq \alpha}^{N_{\Omega+1}} \Omega_{I z}^{(\alpha, \beta)}
$$

This impedance coupling approach is designed to be more stable, as compared to the pressure and velocity coupling approach, due to the introduction of artificial damping into the numerical system [141]. The approach is inspired by the finite element tearing and interconnecting method [64] and the ultra weak variational formulation [126], which apply a similar coupling approach. Pluymers [138] shows that choosing $\bar{Z}_{\text {int }}$ to be the characteristic fluid impedance $\rho c$ is beneficial for the convergence rate.

\subsubsection{Wave function selection}

The time-harmonic acoustic pressure field $p^{(\alpha)}(\mathbf{r})$ in an acoustic subdomain $\Omega^{(\alpha)}$ is approximated as solution expansion $\hat{p}^{(\alpha)}(\mathbf{r})$

$$
p^{(\alpha)}(\mathbf{r}) \simeq \hat{p}^{(\alpha)}(\mathbf{r})=\sum_{w=1}^{n_{w}^{(\alpha)}} p_{w}^{(\alpha)} \Phi_{w}^{(\alpha)}(\mathbf{r})+\hat{p}_{q}^{(\alpha)}(\mathbf{r})=\boldsymbol{\Phi}^{(\alpha)}(\mathbf{r}) \mathbf{p}_{\mathbf{w}}^{(\alpha)}+\hat{p}_{q}^{(\alpha)}(\mathbf{r})
$$

The wave function contributions $p_{w}^{(\alpha)}$ are the weighting factors for each of the selected wave functions $\Phi_{w}^{(\alpha)}$. Together they form the vector of degrees of freedom $\mathbf{p}_{\mathbf{w}}^{(\alpha)}$. The corresponding a priori defined wave functions are collected in the row vector $\boldsymbol{\Phi}^{(\alpha)}$. The set of all $n_{W}=\sum_{\alpha=1}^{N_{\Omega}+1} n_{w}^{(\alpha)}$ acoustic wave function contributions $p_{w}$ is collected in the column vector $\mathbf{p}_{\mathbf{w}}$, while the row vector $\boldsymbol{\Phi}$ contains all $n_{W}$ wave functions.

In $(67) \hat{p}_{q}^{(\alpha)}$ represents a particular solution resulting from the acoustic source term $q^{(\alpha)}$ in the inhomogeneous Helmholtz equation (2). It is the free-field solution of a cylindrical source

$$
\hat{p}_{q}^{(\alpha)}(x, y)=\frac{\rho \omega Q^{(\alpha)}}{4} H_{0}^{2}\left(k r_{q}^{(\alpha)}\right)
$$

with $Q^{(\alpha)}$ the source strength

$$
Q^{(\alpha)}=\int_{\Omega^{(\alpha)}} q^{(\alpha)} \mathrm{d} \Omega
$$

with

$$
r_{q}^{(\alpha)}=\left\|\mathbf{r}-\mathbf{r}_{\mathbf{q}}^{(\alpha)}\right\|
$$

and with $H_{0}^{2}$ the zero-order Hankel function of the second kind. 
The definition of the wave functions $\Phi_{w}^{(\alpha)}(\mathbf{r})$ in (67) depends on the topology of the associated subdomain $\Omega^{(\alpha)}$. Abstraction is made from the transformations between the local subdomain coordinate systems, in which the wave functions are defined, and the global coordinate system. As a result, $\Phi_{w}^{(\alpha)}(\mathbf{r})$ corresponds to the evaluation of the wave function in position $\mathbf{r}$ but transformed to the correct subdomain coordinate system.

\section{Wave functions within a bounded subdomain}

Each acoustic wave function $\Phi_{w}^{(\alpha)}(\mathbf{r})$ satisfies exactly the homogeneous Helmholtz equation (2). Two types of wave functions are distinguished, the $r$ - and the s-set

$$
\sum_{w=1}^{n_{w}^{(\alpha)}} p_{w}^{(\alpha)} \Phi_{w}^{(\alpha)}(\mathbf{r})=\sum_{w_{r}=1}^{n_{w_{r}}^{(\alpha)}} p_{w_{r}}{ }^{(\alpha)} \Phi_{w_{r}}^{(\alpha)}(\mathbf{r})+\sum_{w_{s}=1}^{n_{w_{s}}^{(\alpha)}} p_{w_{s}}{ }^{(\alpha)} \Phi_{w_{s}}^{(\alpha)}(\mathbf{r})
$$

with $n_{w}^{(\alpha)}=n_{w_{r}}^{(\alpha)}+n_{w_{s}}^{(\alpha)}$. The wave functions are defined as

$$
\Phi_{w}^{(\alpha)}(\mathbf{r}(x, y))=\left\{\begin{array}{c}
\Phi_{w_{r}}^{(\alpha)}(x, y)=\cos \left(k_{x w_{r}}^{(\alpha)} x\right) e^{-j k_{y w_{r}}^{(\alpha)} y} \\
\Phi_{w_{s}}^{(\alpha)}(x, y)=e^{-j k_{x w_{s}}^{(\alpha)} x} \cos \left(k_{y w_{s}}^{(\alpha)} y\right)
\end{array} .\right.
$$

The only requirement for the wave functions (72) to be exact solutions of (2) is

$$
\left(k_{x w_{r}}^{(\alpha)}\right)^{2}+\left(k_{y w_{r}}^{(\alpha)}\right)^{2}=\left(k_{x w_{s}}^{(\alpha)}\right)^{2}+\left(k_{y w_{s}}^{(\alpha)}\right)^{2}=k^{2} .
$$

As a result, an infinite number of wave functions (72) can be defined for expansion (67). Desmet [136] proposes to select the following wave number components

$$
\begin{aligned}
& \left(k_{x w_{r}}^{(\alpha)}, k_{y w_{r}}^{(\alpha)}\right)=\left(\frac{w_{1}^{(\alpha)} \pi}{L_{x}^{(\alpha)}}, \pm \sqrt{k^{2}-\left(k_{x w_{r}}^{(\alpha)}\right)^{2}}\right), \\
& \left(k_{x w_{s}}^{(\alpha)}, k_{y w_{s}}^{(\alpha)}\right)=\left( \pm \sqrt{k^{2}-\left(k_{y w_{s}}^{(\alpha)}\right)^{2}}, \frac{w_{2}^{(\alpha)} \pi}{L_{y}^{(\alpha)}}\right) .
\end{aligned}
$$

with $w_{1}^{(\alpha)}$ and $w_{2}^{(\alpha)}=0,1,2, \cdots$. The dimensions $L_{x}^{(\alpha)}$ and $L_{y}^{(\alpha)}$ represent the dimensions of the (smallest) bounding rectangle, circumscribing the considered subdomain.

In order to allow the WBM to be implemented in a computer code, the number $n_{a}$ of Trefftz basis functions $\Phi_{a}$ needs to truncated by assigning finite values to the truncation numbers $n_{\bullet}$. These values may depend for example as follows on the physical wave number $k$ and the dimensions of the bounding rectangle

$$
\frac{n_{r}}{L_{x}} \approx \frac{n_{s}}{L_{y}} \geq T \frac{k}{\pi}
$$

where $T$ represents a truncation parameter, which is user defined. Desmet [136] selects $T=2$, which states that the wavelength $\lambda_{\min }$ of the highest oscillating wave function in the set of basis functions $\Phi_{a}$ is not larger than half the characteristic wavelength $\lambda=2 \pi / k$ of the acoustic problem $\left(\lambda_{\min } \leq \frac{1}{2} \lambda\right)$.

\section{Wave functions within the unbounded subdomain}

Ihlenburg [4] and Herrera [118] show that the following expansion, exterior to a circular 
truncation curve with radius $R$, converges for $n_{u} \rightarrow \infty$.

$$
p_{e}(r, \theta) \simeq \widehat{p}_{e}(r, \theta)=p_{e, c 0} H_{0}^{(2)}(k r)+\sum_{n=1}^{n_{u}} p_{e, c n} H_{n}^{(2)}(k r) \cos (n \theta)+p_{e, s n} H_{n}^{(2)}(k r) \sin (n \theta)
$$

with $r$ and $\theta$ polar coordinates. $H_{n}^{(2)}$ is the $n$-th order Hankel function of the second kind. The contributions $p_{e, c 0}, p_{e, c n}$ and $p_{e, s n}$ are the unknowns.

The applied wave functions correspond to the analytical solution of a Neumann problem for the exterior pressure field of an infinitely long cylinder of radius $R$.

\subsubsection{Construction of the wave model}

With the use of the proposed pressure expansions (71) and (77), the Helmholtz equation (2) and the Sommerfeld radiation condition (4) are always exactly satisfied, irrespective of the values of the unknown wave function contributions. These contributions are merely determined by the acoustic boundary and continuity conditions.

Due to the partitioning of the acoustic problem domain $\Omega$ into $N_{\Omega}+1$ acoustic subdomains $\Omega^{(\alpha)}$, continuity conditions $(61)$ or $(65)$ along the subdomain interfaces $\Gamma_{I}^{(\alpha, \beta)}$ must be taken into account, in addition to the problem boundary conditions (3). The unknown wave function contribution factors are merely determined by these boundary and continuity conditions.

Since both the boundary conditions and the continuity conditions are defined at an infinite number of boundary positions, while only finite sized prediction models are amenable to numerical implementation, the boundary and the continuity conditions are, for each subdomain, transformed into a weighted residual formulation. The residual error functions are defined as

- residual error functions of the boundary conditions

$$
\begin{array}{lll}
\mathbf{r} \in \Gamma_{v}^{(\alpha)} \quad: \quad R_{v}^{(\alpha)}(\mathbf{r})=\mathcal{L}_{v}^{(\alpha)}\left(\hat{p}^{(\alpha)}(\mathbf{r})\right)-\bar{v}_{n}(\mathbf{r}) \\
\mathbf{r} \in \Gamma_{Z}^{(\alpha)} \quad: \quad R_{Z}^{(\alpha)}(\mathbf{r})=\mathcal{L}_{v}^{(\alpha)}\left(\hat{p}^{(\alpha)}(\mathbf{r})\right)-\hat{p}^{(\alpha)}(\mathbf{r}) / \bar{Z}_{n}(\mathbf{r}) \\
\mathbf{r} \in \Gamma_{p}^{(\alpha)} \quad: \quad R_{p}^{(\alpha)}(\mathbf{r})=\hat{p}^{(\alpha)}(\mathbf{r})-\bar{p}(\mathbf{r})
\end{array}
$$

- residual error functions of the pressure and velocity continuity conditions

$$
\begin{array}{ll}
\mathbf{r} \in \Gamma_{I p}^{(\alpha, \beta)} \quad: \quad R_{I p}^{(\alpha, \beta)}(\mathbf{r})=\hat{p}^{(\alpha)}(\mathbf{r})-\hat{p}^{(\beta)}(\mathbf{r}) \\
\mathbf{r} \in \Gamma_{I v}^{(\alpha, \beta)} \quad: \quad R_{I v}^{(\alpha, \beta)}(\mathbf{r})=\mathcal{L}_{v}^{(\alpha)}\left(\hat{p}^{(\alpha)}(\mathbf{r})\right)+\mathcal{L}_{v}^{(\beta)}\left(\hat{p}^{(\beta)}(\mathbf{r})\right)
\end{array}
$$

- residual error functions of the impedance continuity conditions

$$
\mathbf{r} \in \Gamma_{I z}^{(\alpha, \beta)} \quad: \quad R_{I}^{(\alpha, \beta)}(\mathbf{r})=\mathcal{L}_{e q+}^{(\alpha)}\left(\hat{p}^{(\alpha)}(\mathbf{r})\right)-\mathcal{L}_{\text {eq- }}^{(\beta)}\left(\hat{p}^{(\beta)}(\mathbf{r})\right)
$$

Similar error functions are derived for all $N_{\Omega}+1$ acoustic subdomains. For each subdomain, the error functions are orthogonalized in a complementary way with respect to a weighting 
function $\tilde{p}^{(\alpha)}$ or its derivative. The weighted residual formulation, applying the introduced error functions, is expressed as

$$
\begin{gathered}
\int_{\Gamma_{v}^{(\alpha)}} \tilde{p}^{(\alpha)}(\mathbf{r}) R_{v}^{(\alpha)}(\mathbf{r}) d \Gamma+\int_{\Gamma_{Z}^{(\alpha)}} \tilde{p}^{(\alpha)}(\mathbf{r}) R_{Z}^{(\alpha)}(\mathbf{r}) \mathrm{d} \Gamma-\int_{\Gamma_{p}^{(\alpha)}} \mathcal{L}_{v}^{(\alpha)}\left(\tilde{p}^{(\alpha)}(\mathbf{r})\right) R_{p}^{(\alpha)}(\mathbf{r}) d \Gamma \\
-\sum_{\beta=1, \beta \neq \alpha}^{N_{\Omega+1}} \int_{\Gamma_{I p}^{(\alpha)}} \mathcal{L}_{v}^{(\alpha)}\left(\tilde{p}^{(\alpha)}(\mathbf{r})\right) R_{I p}^{(\alpha, \beta)}(\mathbf{r}) d \Gamma+\sum_{\beta=1, \beta \neq \alpha}^{N_{\Omega+1}} \int_{\Gamma_{I v}^{(\alpha)}} \tilde{p}^{(\alpha)}(\mathbf{r}) R_{I v}^{(\alpha, \beta)}(\mathbf{r}) \mathrm{d} \Gamma \\
+\sum_{\beta=1, \beta \neq \alpha}^{N_{\Omega+1}} \int_{\Gamma_{I z}^{(\alpha, \beta)}} \tilde{p}^{(\alpha)}(\mathbf{r}) R_{I z}^{(\alpha, \beta)}(\mathbf{r}) \mathrm{d} \Gamma=0 .
\end{gathered}
$$

Like in the Galerkin weighting procedure, used in the FEM, the weighting functions $\tilde{p}^{(\alpha)}$ are expanded in terms of the same set of acoustic wave functions used in the pressure expansions (67) and (77)

$$
\tilde{p}^{(\alpha)}(\mathbf{r})=\sum_{a=1}^{n_{a}^{(\alpha)}}{\tilde{p_{a}}}^{(\alpha)} \Phi_{a}^{(\alpha)}(\mathbf{r})=\boldsymbol{\Phi}^{(\alpha)}(\mathbf{r}) \tilde{\mathbf{p}}_{\mathbf{w}}^{(\alpha)} .
$$

Substitution of the pressure expansions (67) and (77) and the weighting function expansion (82) into the weighted residual formulation (81) and imposing that the formulation should hold for any weighting function $\tilde{p}^{(\alpha)}$, yields a set of $n_{w}^{(\alpha)}$ linear equations in the $n_{W}$ unknown wave function contribution factors.

Expressing the weighted residual formulation (81) for each subdomain results in $N_{\Omega}+1$ systems of equations. Combination of these equations yields the acoustic WB model, consisting of $n_{W}$ algebraic equations in the $n_{W}$ unknown wave function contribution factors

$$
\mathbf{A}_{\mathrm{aa}} \mathbf{p}_{\mathrm{w}}=\mathbf{b}
$$

A full description of the WB system matrices is given in appendix A.

\subsubsection{Solution of the wave based model}

After partitioning into convex subdomains, selection of a converging set of wave functions and construction of the WB model, the fourth step in the WB modelling process is solution of the WB matrix equation (83) for the $n_{W}$ wave function contribution factors $p_{w}$.

\subsubsection{Postprocessing}

The fifth and final step in the modelling process is backsubstitution of the resulting wave function contribution factors into the pressure expansions (67), yielding an analytical description of the dynamic pressure field $\hat{p}$ in all subdomains $\Omega^{(\alpha)}$. Evaluation of wave function expansions in response points located inside the subdomains, yields the acoustic pressures at those locations. Higher-order variables, such as acoustic velocities, intensities and power distributions, can be easily obtained by derivation of the wave function expansions.

- The acoustic velocity vector field $\hat{\mathbf{v}}$ is determined proportional to the gradient of the pressure field

$$
\hat{\mathbf{v}}=\frac{j}{\rho \omega}\left(\boldsymbol{\nabla} \boldsymbol{\Phi} \mathbf{p}_{\mathbf{w}}+\nabla \hat{p}_{q}\right)
$$


- The active acoustic intensity vector field $\hat{\mathbf{I}}$, representing the flow of acoustic energy, is determined as

$$
\hat{\mathbf{I}}=\frac{1}{2} \Re\left(\left(\boldsymbol{\Phi} \mathbf{p}_{\mathbf{w}}+\hat{p}_{q}\right)\left(\frac{j}{\rho \omega}\left(\boldsymbol{\nabla} \boldsymbol{\Phi} \mathbf{p}_{\mathbf{w}}+\nabla \hat{p}_{q}\right)\right)^{*}\right)
$$

with $\bullet *$ denoting the complex conjugate and $\Re$ the real part.

- The active acoustic power $\hat{W}$ through a surface $S$ is determined as the integral of the acoustic intensity through the considered surface and is calculated as

$$
\hat{W}=\iint_{S} \frac{1}{2} \Re\left(\left(\boldsymbol{\Phi} \mathbf{p}_{\mathbf{w}}+\hat{p}_{q}\right)\left(\frac{j}{\rho \omega}\left(\boldsymbol{\nabla} \boldsymbol{\Phi} \mathbf{p}_{\mathbf{w}}+\nabla \hat{p}_{q}\right)\right)^{*}\right) \mathbf{n d} S
$$

with $\mathbf{n}$ the normal vector on $S$.

Note that there is no loss of accuracy in the higher-order variables, since the derivatives of wave functions, are wave functions themselves, with identical wavelengths as the primary wave functions.

\subsection{Model properties}

This section discusses the properties of a WB model and compares them with those of conventional element based models, i.e. the FEM and the BEM.

\section{Degrees of freedom}

The degrees of freedom (dofs) in a FE model are the nodal values, being acoustic pressures most commonly. In an acoustic BE model, the dofs are either acoustic pressures and velocities (direct BEM) or single and double layer potentials (indirect BEM). In the WB methodology, the unknown wave function contributions $p_{w}$ form the dofs.

\section{Problem discretization \& approximation functions}

The element based methods require a discretization of the problem domain (FEM) or of the problem boundary (BEM) into small elements. Within these elements approximating shape functions, mostly simple polynomials, are used to describe the dynamic response variables. In order to yield prediction results with reasonable accuracy, element sizes have to decrease with increasing frequency, because wavelengths shorten. Construction of a WB model does not require a problem domain discretization into small elements. The only prerequisite is a partitioning into convex subdomains. However, these subdomains can be large and their sizes are independent of frequency, since the wave functions, which are used to describe the dynamic response variables inside the subdomains, are exact solutions of the governing dynamic equations. With increasing frequencies, the number of wave functions is increased.

\section{Accuracy of derived variables}

Due to the fact that the primary response variables (i.e. the pressure) in the FEM are most commonly approximated with simple polynomial shape functions, the higher-order derived quantities (i.e. the acoustic velocity, acoustic intensity and acoustic power) are less accurate than the primary ones. In the BEM, a boundary integral formulation preserves the same accuracy for both the primary and the derived variables, so there is no additional loss of accuracy. Also for the WBM, there is no additional loss of accuracy because the derivatives of the wave functions are wave functions as well, with identical wavelengths.

\section{System matrix properties}

The system matrices in the FEM are large and sparsely populated with real coefficients 
B. Pluymers, B. van Hal, D. Vandepitte and W. Desmet

(except when complex model properties are introduced). They have a banded structure, are symmetric and can be decomposed into frequency independent submatrices. All of these properties allow computationally efficient storage and solution of the FE system of equations. The BEM yields fully populated matrices with complex coefficients. These matrices are smaller than the system matrices of FE models for comparable problems, but the BE matrices are frequency dependent and non-symmetric for the direct BEM and symmetric for the indirect BEM. The WB system matrices have similar properties as the matrices in the direct BEM, in that they are fully populated with complex coefficients, non-symmetric and frequency dependent. However, WB system matrices are smaller than BE system matrices.

\section{Problem geometric complexity}

Because of the fine discretization of the element based methods, these methods have almost no restrictions regarding the geometric complexity of the considered problem domains. The only requirement regarding the problem domain geometry for the WBM to converge, is convexity of the considered subdomains. However, since the WB subdomains are large and have a relatively simple shape, problems with a complex geometry are hard to model, unless a large number of subdomains are introduced. This would have a negative effect on the method's computational efficiency.

When unbounded domains come into play, like for instance for acoustic radiation calculations, the fine discretization of the problem domain in the FE methodology prevents the FEM to tackle this kind of problems. Work-arounds are the application of approximating impedance boundary conditions on a truncation surface or coupling of a truncated FE model with infinite elements. Due to the specific shape of the Green functions applied in the BEM, the method inherently satisfies the Sommerfeld radiation condition and can therefore tackle problems involving unbounded domains. Through the appropriate selection of wave functions which satisfy the Sommerfeld radiation condition, the WBM is able to tackle unbounded problems.

\section{Computational performance \& frequency range applicability}

Construction of the WB models results from tedious numerical integrations. Because the WB method, like any Trefftz based method, yields ill-conditioned system matrices [122, 158], the numerical integrations must be performed carefully, making sure that a sufficiently high accuracy in determining the matrix coefficients is obtained. As a result, construction of the WB matrices is computationally more demanding than construction of FE matrices, whose coefficients result from integrations of simple polynomial shape functions, and even than construction of $\mathrm{BE}$ matrices. However, because of the large model, and resulting matrix size, of the FE and BE models, as compared to the WB models, solution of the element based models is computationally much more demanding than solution of the WB models. Also the convergence rate of the WBM is higher than the convergence rate of the element based methods.

The higher convergence rate, combined with the small model size, make it possible for the WBM to tackle problems at higher frequencies than the element based methods, before the same limitations regarding computational resources are encountered.

\section{NUMERICAL VALIDATIONS OF THE WAVE BASED METHOD}

This section applies the Trefftz-based WBM for the analysis of both an interior and an exterior $2 \mathrm{D}$ acoustic problem. In both cases, the WBM shows to be computationally more efficient than conventional element based methods. 


\subsection{Interior acoustics: a car cavity}

\subsubsection{Problem description}

Figure 12 shows a 2D car cavity with a fairly complex geometry, especially due to the modelling of the front seat as a rigid object. The cavity is filled with air $\left(\rho_{a}=1.225 \mathrm{~kg} / \mathrm{m}^{3}\right.$, $c=340 \mathrm{~m} / \mathrm{s}$ ) and it has the characteristic dimensions $L_{x} \approx 2.7 \mathrm{~m}$ and $L_{y} \approx 1.1 \mathrm{~m}$. The main part of the domain boundary is rigid $\left(\bar{v}_{n}=0 \mathrm{~m} / \mathrm{s}\right)$. An impedance boundary condition is imposed at the roof with $\bar{Z}=2000 \mathrm{~Pa} . \mathrm{s} / \mathrm{m}$, which introduces damping in the acoustic system. A unit normal velocity distribution $\bar{v}_{n}=1 \mathrm{~m} / \mathrm{s}$ at the fire wall excites the $2 \mathrm{D}$ car cavity. This $2 \mathrm{D}$ example is used in several publications on enhanced deterministic methods for interior acoustic problems, a.o. [43, 84, 159].

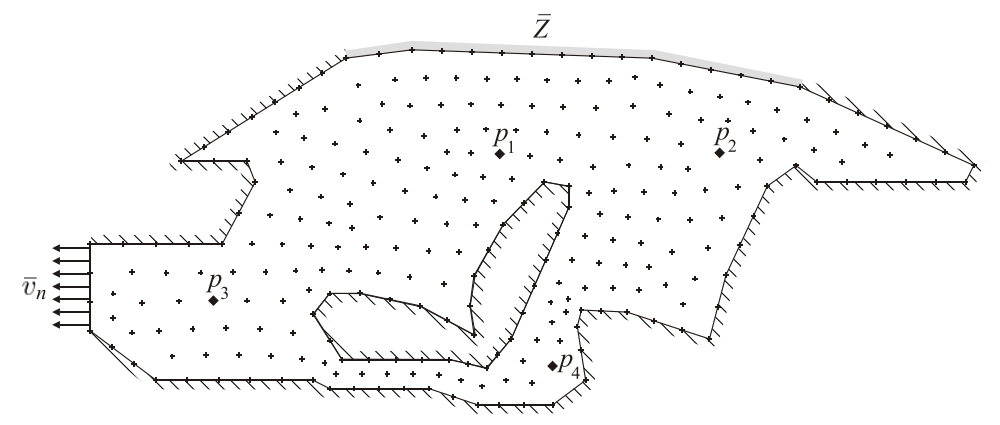

Figure 12: 2D acoustic car cavity

\subsubsection{Numerical models}

Van Hal [128] has shown that it is beneficial for the WBM's convergence rate, if the subdomains, which are created by the partitioning preceding the construction of the WB model, are regularly shaped and rectangular. Clearly, a partitioning in only such regularly shaped subdomains is not possible and thus an optimal balance between the number of subdomains and their shape needs to be found. Either one selects a relatively small number of irregularly shaped subdomains or one selects a large number of more regularly shaped subdomains. Several domain partitions are applied in this numerical example. In all the considered numerical WB models, pressure and velocity continuity conditions are applied at the resulting interfaces.

Figure 13 shows five WB model configurations and one FE model. These WB model configurations are listed below with an increasing level of regularity of the subdomain geometries.

- WB model I contains a relatively small number of 18 subdomains, which are all irregularly shaped having 3 to 7 edges. The area ratio between the largest and the smallest subdomain is high.

- WB model II contains 26 subdomains with more regular geometries. Most subdomains have two parallel edges and corner points where edges intersect at (nearly) $90^{\circ}$.

- WB model III is derived from WB model II. It contains 36 more regularly shaped subdomains by splitting several subdomains such that most subdomains have 4 edges.

- WB model IV contains 30 subdomains which have (nearly) rectangular shapes. In other words, most of these subdomains are more regularly shaped than the subdomains in the WB models III and IV. 

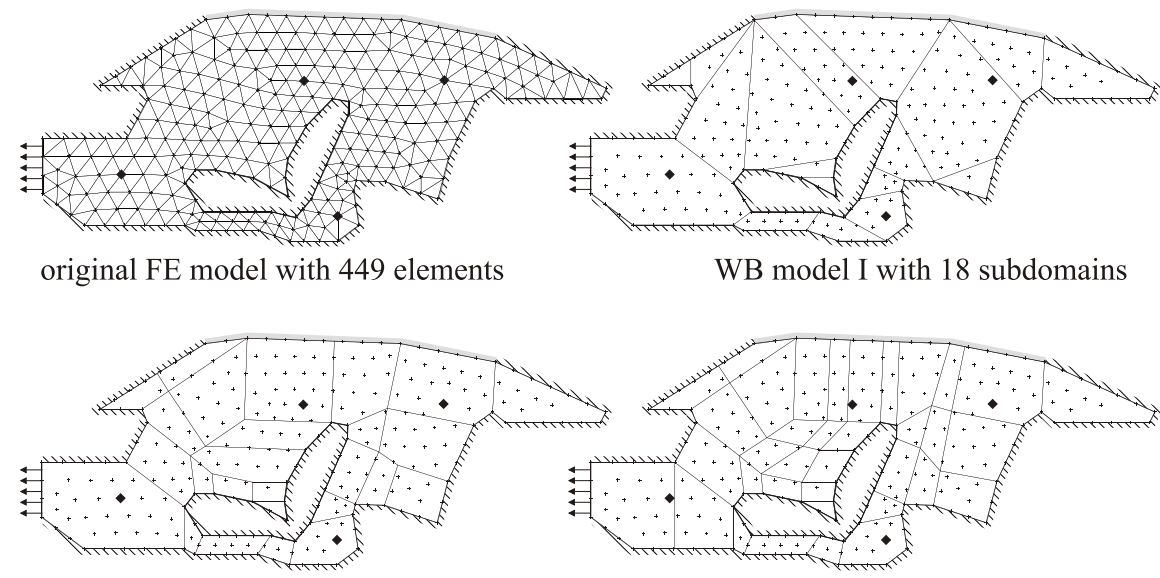

WB model II with 26 subdomains

WB model III with 36 subdomains

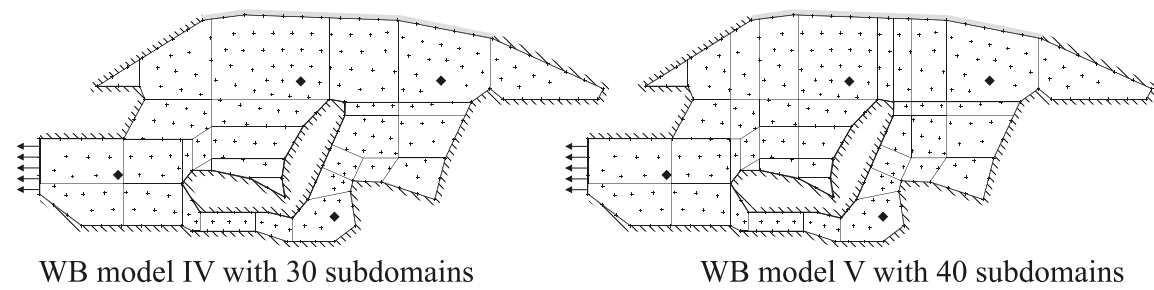

Figure 13: Original FE model and various WB model configurations of 2D car cavity

- WB model V is derived from WB model IV. It contains 40 (nearly) rectangular subdomains by splitting several subdomains such that most subdomains have 4 edges.

Two FE models, which are used in an FRF analysis, are derived from the original FE model in figure 13.

- A coarse FE model of 1796 linear elements and 1007 DOFs results from subdividing each triangular element of the original $\mathrm{FE}$ model in 4 triangular elements. It is applicable up to $f_{\max } \approx 270 \mathrm{~Hz}$ according to rule (21) with $L=2.7 \mathrm{~m}$ and $h=$ $0.054 \mathrm{~m}$.

- A fine FE model of $161642^{\text {nd }}$-order elements and 32982 DOFs results from subdividing each triangular element of the original FE model in 36 triangular elements. This model serves as reference model up to $f_{\max } \approx 1900 \mathrm{~Hz}$ according to dispersion rule for quadratic FE described by Ihlenburg [4] with $L=2.7 \mathrm{~m}$ and $h=0.018 \mathrm{~m}$.

\subsubsection{Evaluation of the performance of the WBM}

Figure 14 shows the predictions of the pressure response $p$ at position $p_{1}$ indicated in figure 12. The first plot illustrates the effect of the pollution error on the FE pressure response prediction $\hat{p}$. The visual inspection on the current scale shows that the FE prediction starts to deviate from the reference near $400 \mathrm{~Hz}$. This is more in agreement with rule (21), which provides $f_{\max } \approx 270 \mathrm{~Hz}$, than with the 'rule of thumb' $(k \cdot h=1)$, which provides $f_{\max } \approx 1000 \mathrm{~Hz}$.

The WB results in figure 14 are obtained by using a truncation parameter of $T=2$. These results confirm that the WBM does not suffer from pollution errors. Even in the upper part of the frequency range of interest, the WB predictions coincide with the reference 


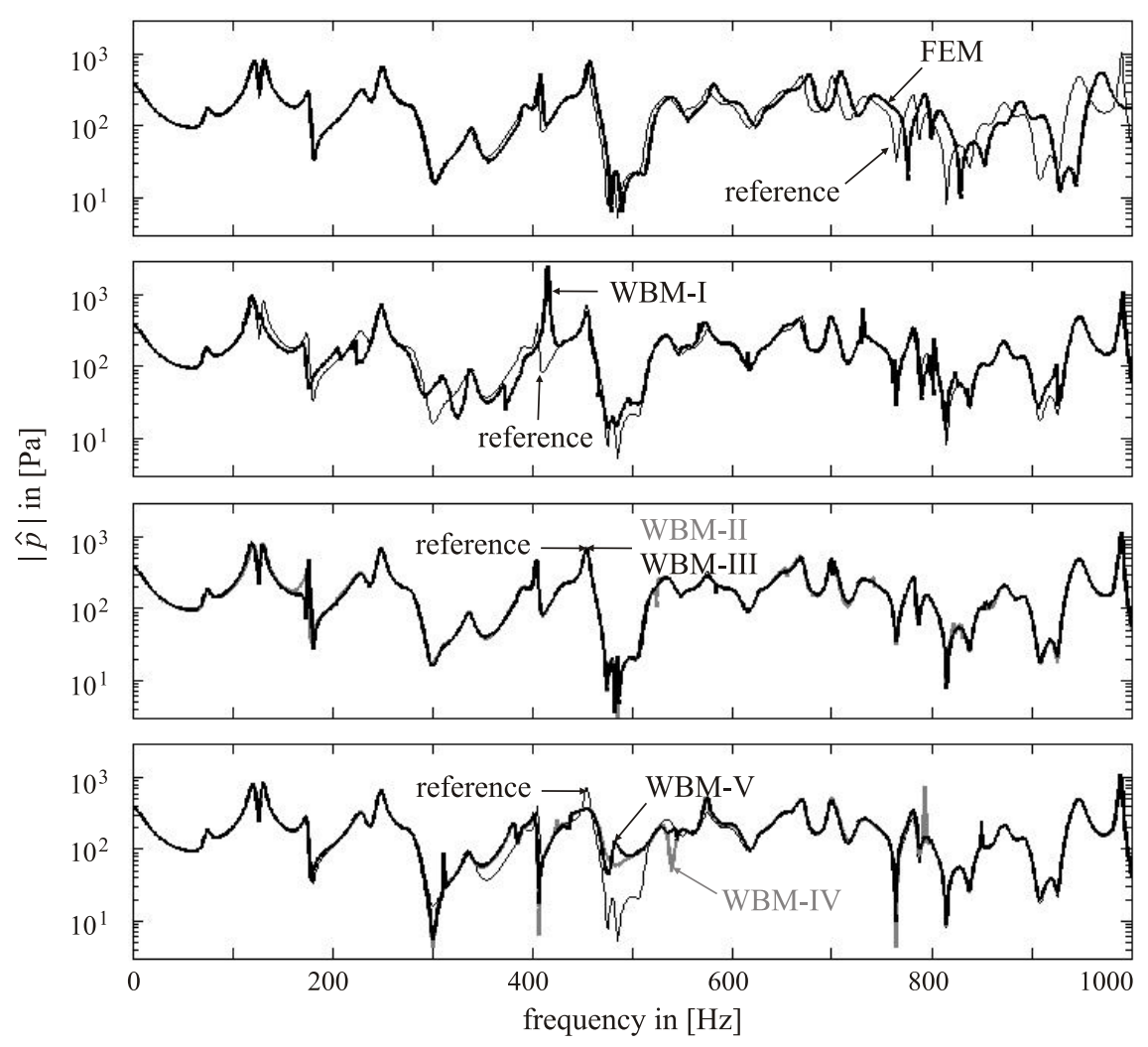

Figure 14: Pressure response prediction for point $p_{1}$ in figure 12

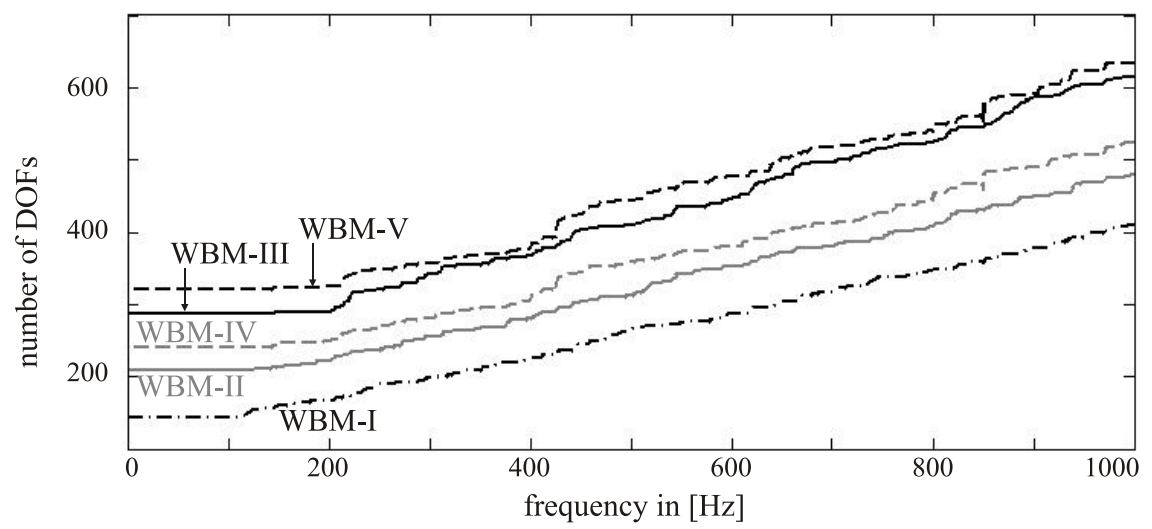

Figure 15: WB model sizes with truncation parameter $T=2$ 
solution more or less. However, this numerical validation example reveals two aspects of the WB pressure response prediction $\hat{p}$.

- Firstly, the influence of the domain subdivision can already be observed on the current scale of the graphs.

- Secondly, below $\approx 600 \mathrm{~Hz}$ some WB results have not converged yet. Especially, the results for WB model I show some deviation from the reference and, in less extent, the results for WB models IV and V.

These phenomena are observed in an a posteriori error assessment too.

An a posteriori error assessment is performed at $215 \mathrm{~Hz}, 525 \mathrm{~Hz}$ and $955 \mathrm{~Hz}$. Figure 16 shows the pressure distributions at those frequencies obtained with WB model I with a truncation parameter $T=2$. These pressure distributions do not show irregularities at the interfaces between two subdomains on the current scale of the graphs. This indicates that WB model I provides reliable results for the considered excitation frequencies with a truncation parameter $T=2$.

Figure 17 shows the averaged relative pressure $\langle\epsilon\rangle$ defined as

$$
\langle\epsilon\rangle=\sum_{j=1}^{n_{\mathrm{rp}}} \frac{A_{j} \epsilon_{j}}{A} \text { with } A=\sum_{j=1}^{n_{\mathrm{rp}}} A_{j}
$$

real part of pressure distribution in $[\mathrm{Pa}] \quad$ imaginary part of pressure distribution in $[\mathrm{Pa}]$
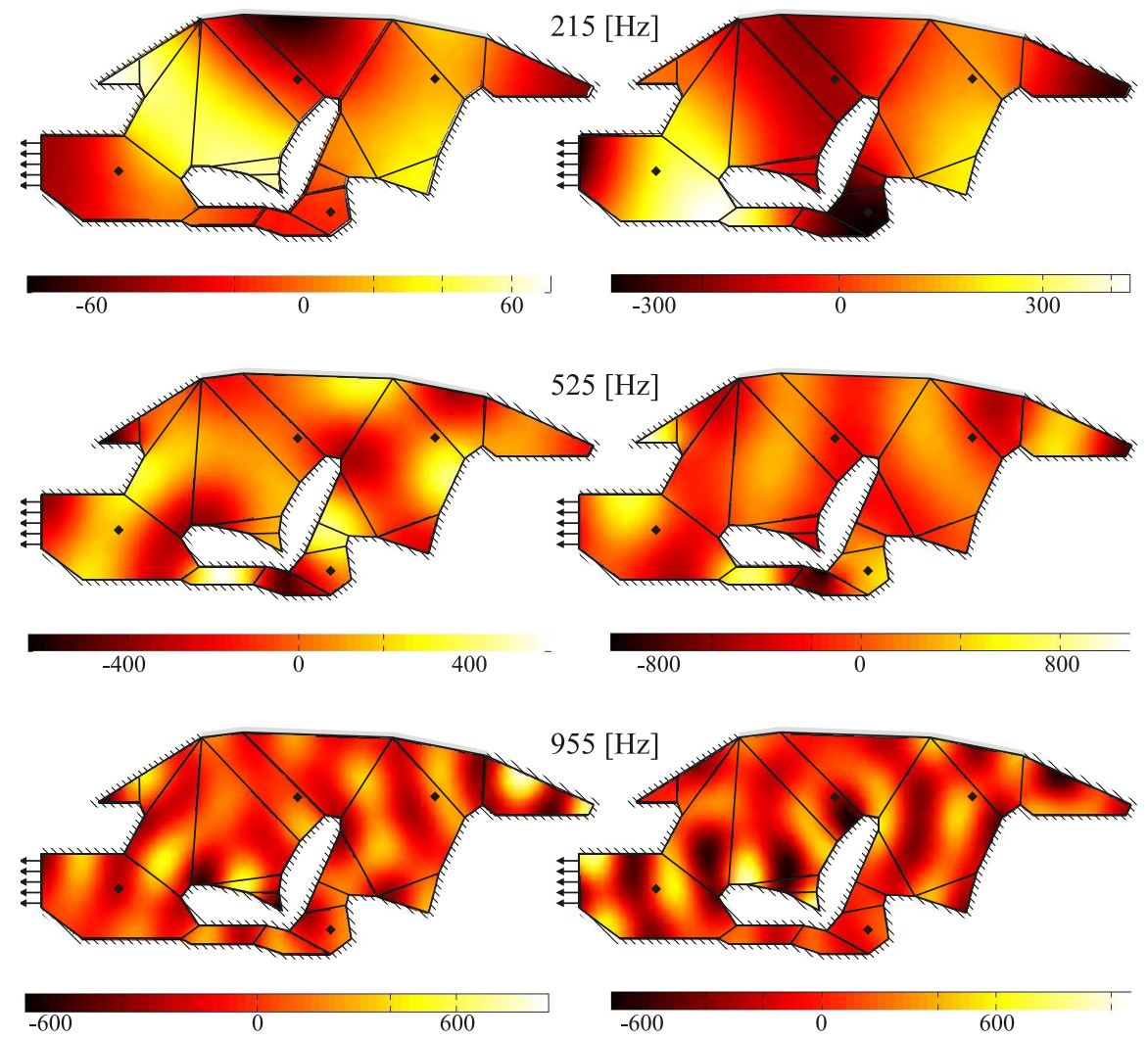

Figure 16: Pressure distributions obtained with WB model I 
which is based on the relative pressure error $\epsilon$

$$
\epsilon_{j}=\frac{\left\|\hat{p}_{j}-\hat{p}_{\text {ref }, j}\right\|}{\left\|\hat{p}_{\text {ref }, j}\right\|} .
$$

where $n_{\mathrm{rp}}$ represents the number of response points, $A_{j}$ the area surrounding response point $j$ and $A$ the area of the problem domain $\Omega$, for the $n_{\mathrm{rp}}=279$ response points indicated by + in figure 12. The following phenomena are observed.

- The computational efficiency depends on the frequency of interest.

- At the high frequency of $955 \mathrm{~Hz}$, the WBM exhibits enhanced convergence properties, especially compared with FEM based on linear elements.

- At $525 \mathrm{~Hz}$, the computational efficiency of the WBM is not as pronounced as at $955 \mathrm{~Hz}$. However, the WBM is still more efficient than the FEM based on linear elements since, on average, the same accuracy is obtained with smaller WB models.

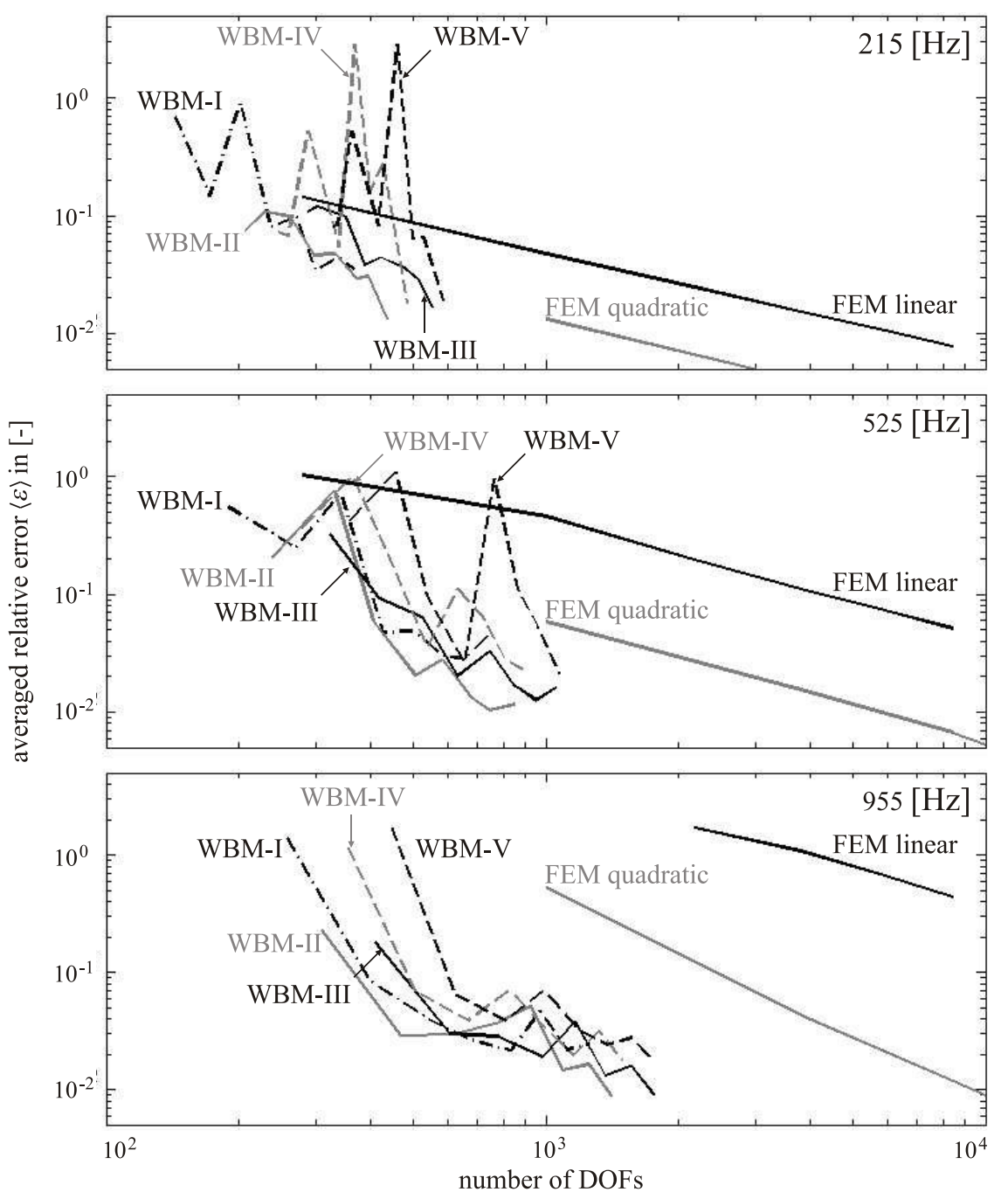

Figure 17: Convergence 
- At the low frequency of $215 \mathrm{~Hz}$, the computational efficiency of the WBM and the FEM based on linear elements are comparable.

In case of interior acoustic problems with geometrically complex boundaries, the FEM is best suited for low frequency applications whereas the WBM performs better at higher frequencies.

- The WBM convergence curves show an oscillatory behaviour which differs for each WB model configuration. Especially at $215 \mathrm{~Hz}$ and $525 \mathrm{~Hz}$, severe oscillations in the convergence occur for WB models I, IV and V. Apparently, WB models II and III are most robust for the considered frequencies. This suggest that the optimal balance between the number of subdomains and their shape is obtained by

- minimizing the number of subdomains

- while avoiding the occurrence of highly irregularly subdomains.

In order to improve the low-frequency results, a minimal value for the truncation numbers $n_{r}$ and $n_{s}$ in (76) can be imposed [128].

\subsection{Exterior acoustics: a bass-reflex loudspeaker}

\subsubsection{Problem definition}

In order to illustrate the ability of the WBM to analyze more complex 2D unbounded problems, a bass-reflex loudspeaker, as shown in figure 18, is considered. A unit normal line force $F$ is applied at the centre of the loudspeaker membrane $\left(E=70.10^{9} \mathrm{~N} / \mathrm{m}^{2}\right.$, $\left.\rho_{s}=700 \mathrm{~kg} / \mathrm{m}^{3}, \nu=0.3, t=3 \mathrm{~mm}\right)$. The membrane edges are clamped. The loudspeaker is surrounded with air $\left(c=340 \mathrm{~m} / \mathrm{s}, \rho=1.225 \mathrm{~kg} / \mathrm{m}^{3}\right)$.

\subsubsection{Numerical models}

To compare the performances of the WBM and the existing element based techniques, several coupled $\mathrm{FE} /$ indirect $\mathrm{BE}$ and $\mathrm{FE} /\left(8^{\text {th }}\right.$ order conjugated) IE models of the considered problem have been solved using LMS/Sysnoise Rev5.5. The structural FE meshes consist of third-order 2-noded plate elements, the acoustic BE meshes of 2-noded linear fluid elements and the acoustic FE meshes of 3- and 4-noded linear fluid elements. Tables 1 and 2 show the number of elements used to model the considered loudspeaker problem.

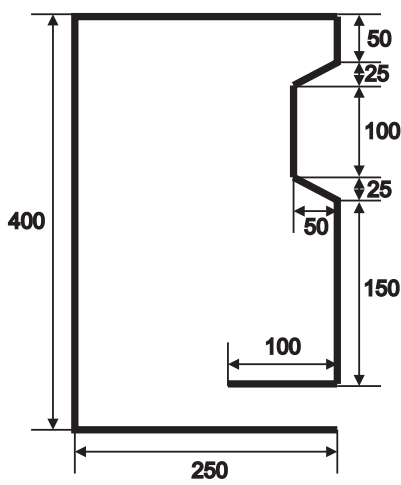

Figure 18: Dimensions (in $m m$ ) of a bass-reflex loudspeaker

For the WB models a circular truncation curve with radius $R=0.5 \mathrm{~m}$ is applied. Figure 19 shows the partitioning into 11 convex subdomains of the region interior to the truncation curve. Equivalent normal velocity continuity conditions are applied at the resulting 
interfaces with a coupling factor $\bar{Z}_{i n t}=\rho c$. Table 3 lists the WB model information at $5000 \mathrm{~Hz}$.

\begin{tabular}{|c|c|c|c|c|}
\hline $\begin{array}{c}\sharp \text { acoustic FE } \\
\text { inside cavity }\end{array}$ & $\begin{array}{c}\sharp \text { acoustic FE } \\
\text { outside cavity }\end{array}$ & $\begin{array}{c}\sharp \text { structural } \\
\text { FE }\end{array}$ & $\sharp$ IE & $\begin{array}{c}\text { total } \\
\sharp \text { dofs }\end{array}$ \\
\hline 2835 & 7786 & 34 & 312 & 14567 \\
4832 & 15973 & 68 & 394 & 25031 \\
9492 & 26525 & 136 & 524 & 42552 \\
\hline
\end{tabular}

Table 1: FE/IE model information

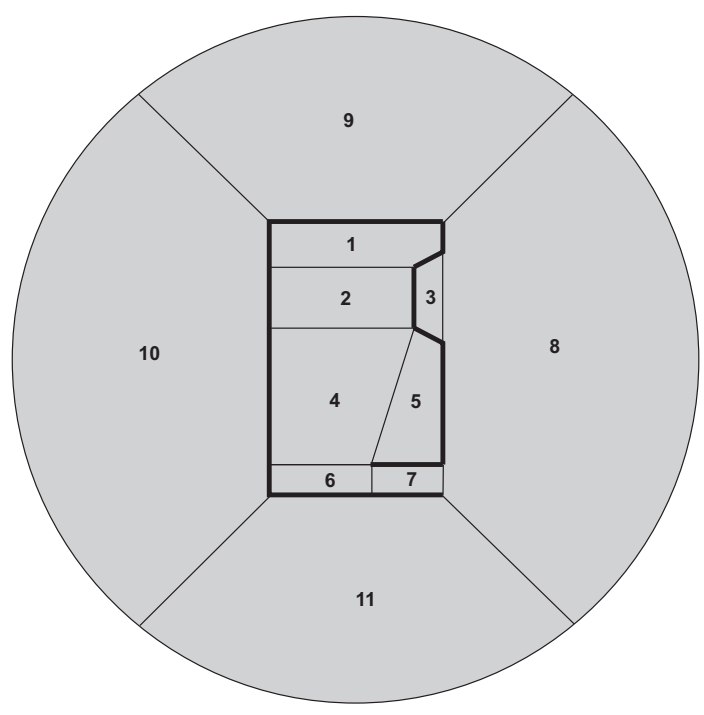

Figure 19: WB partitioning

\begin{tabular}{|c|c|c|}
$\sharp$ ac. BE & $\sharp$ str. BE & tot. $\sharp$ dofs \\
\hline 472 & 34 & 593 \\
944 & 68 & 1167 \\
1888 & 136 & 2315 \\
3776 & 272 & 4611 \\
\hline
\end{tabular}

Table 2: BE model information

\begin{tabular}{|c|c|c|}
$\sharp$ ac. WB dofs & $\sharp$ str. WB dofs & tot. $\sharp$ WB dofs \\
\hline 131 & 4 & 135 \\
195 & 4 & 199 \\
327 & 4 & 331 \\
587 & 4 & 591 \\
853 & 4 & 857 \\
\hline
\end{tabular}

Table 3: WB model information 


\subsubsection{Evaluation of the performance of the WBM}

Figures 20 and 21 show the calculated pressure field and the calculated active intensity field at $120 \mathrm{~Hz}$. These results are obtained with a wave model which consists of 495 wave functions. The pressure contour plots show that the rigid boundary conditions are correctly taken into account by the WBM since the pressure contour lines are perpendicular to the rigid walls. Also, no pressure field discontinuities are observed, which indicates that the continuity conditions at the subdomain interfaces and the infinite domain interfaces are correctly taken into account. Figure 21 shows that active intensity flows from both the membrane and the reflex-channel towards infinity which clearly illustrates the working principle of a bass-reflex channel. For the given loudspeaker dimensions, the considered frequency of $120 \mathrm{~Hz}$ indeed corresponds to the reflex-frequency of the loudspeaker.

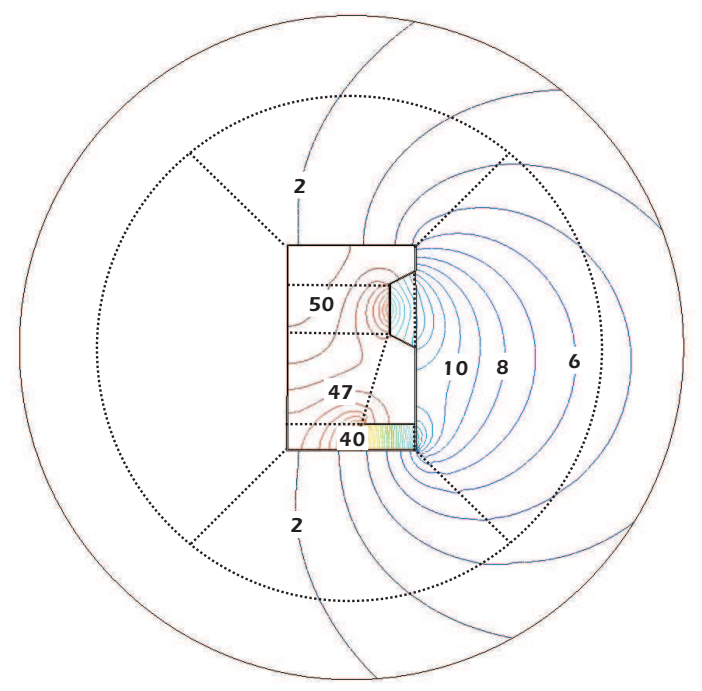

Figure 20: Contour plot of the pressure amplitudes at $120 \mathrm{~Hz}\left[10^{-4} \mathrm{~Pa}\right]$

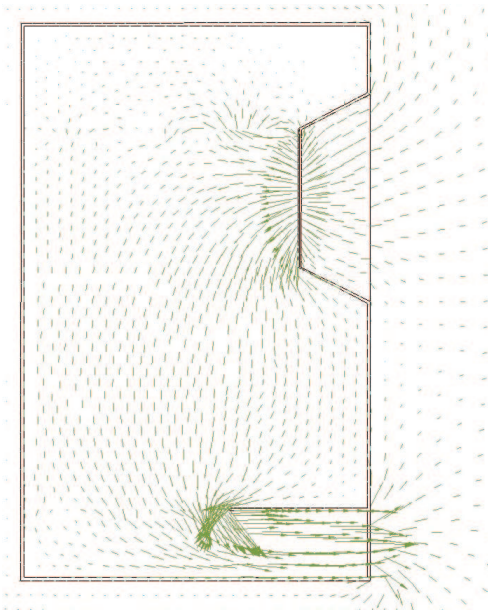

Figure 21: Vector plot of the active intensity at $120 \mathrm{~Hz}$

Figure 22 plots the relative prediction error for the radiated sound power $\mathrm{W}$ at $5000 \mathrm{~Hz}$ against the CPU time needed for a direct response calculation at one frequency on an Intel 
Pentium 4 computer system (1.8 GHz, 1 Gb RAM) running a Windows XP-Professional operating system. The indicated CPU times for the WBM and the FE/BE models include both the times for construction of the model and for solution of the resulting matrix equation since the matrices are frequency dependent. This is in contrast with the frequency independent $\mathrm{FE}$ /conjugated IE models where only the solution time is taken into account. The WBM exhibits a beneficial convergence rate, compared with the element based techniques.

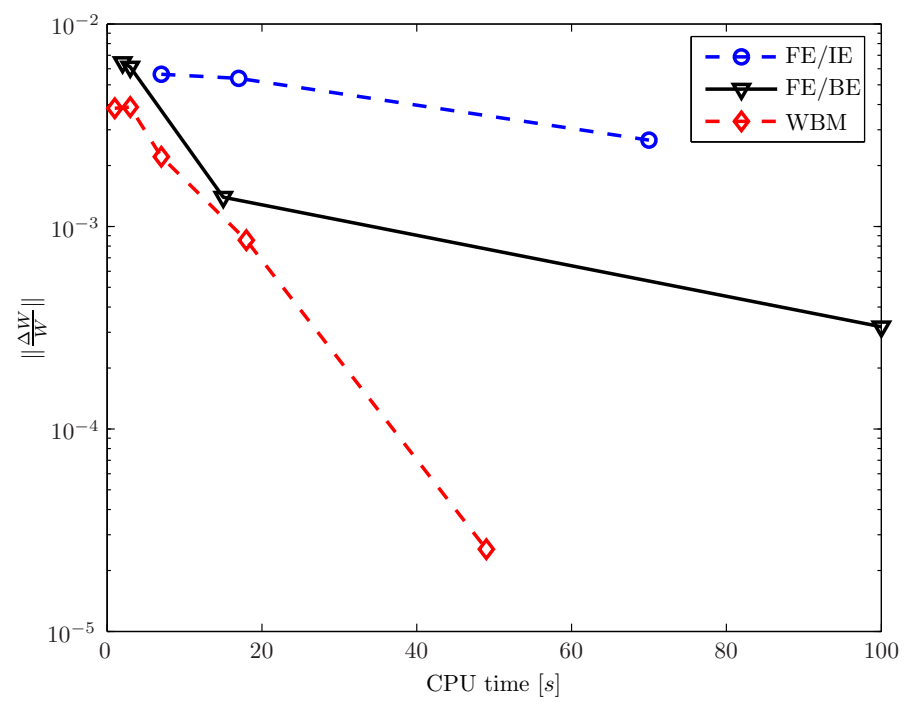

Figure 22: Power convergence curves at $5000 \mathrm{~Hz}$

To increase the accuracy of the FE/IE models, a finer element discretization is required. However, since the artificial boundary, which truncates the unbounded domain, was chosen to be a circle, the greater part of the acoustic finite elements are needed to model the enclosed area between the artificial truncation boundary surface and the cavity, which is not very efficient and which leads to prohibitively large calculations. A more efficient way of modelling the problem would be to use a more close-fitting truncation boundary, such as for instance an elliptical boundary, so that the area between the truncation boundary and the problem boundary becomes much smaller.

\section{CONCLUSIONS}

This paper gives an overview of element based and Trefftz-based modelling methods for the analysis of time-harmonic acoustic problems. Element based techniques, such as the FEM and the BEM, are commonly applied. These methods discretize the considered problem domain or its boundary into small elements and make use of locally defined, approximating shape functions to describe the dynamic response. Due to the approximating nature of the applied shape functions, the numerical solution suffers from numerical dispersion. For a fixed element discretization, the associated dispersion errors grow with increasing frequency. In order to maintain a reasonable prediction accuracy at higher frequencies, the element discretization needs to be refined. This results in (prohibitively) large matrix equations and associated computational resources, such that the practical use of the FEM and the BEM is restricted to low-frequency applications.

A vast amount of research is on-going in the development of extensions and enhancements of the FEM and the BEM, to enlarge their practical applicability towards problems 
at higher frequencies. Some extensions focus on the optimization of the numerical processes, while others try to reduce the dispersion error by means of a modified integral problem formulation. Other classes of extended methods deal with the application of novel or enriched sets of approximation functions. In spite of these numerous attempts to develop methods applicable for tackling problems at higher frequencies, no mature method has emerged yet, which is applicable for real-life engineering applications. Most of the novel methods have been applied so far on academic cases only.

In parallel with the investigations regarding the element based methods, an alternative family of numerical methods, based on a Trefftz approach, is researched. Trefftz-based methods apply approximation functions which exactly satisfy the governing differential equations, resulting in small, computationally efficient numerical models. Although most of the Trefftz-based methods also have characteristics limiting their practical application range, one of these methods has proven to be robust and applicable for the analysis of general acoustic problems. This so-called WBM shows high potential for tackling real-life problems in at high frequencies.

The basic formulations of the WBM for 2D acoustic problems are discussed and the application of the method is illustrated by means of an interior and an exterior numerical validation case. It is shown that the method provides accurate predictions and that it outperformes the conventional element based methods, especially when going to higher frequencies.

It is also shown that, in order to fully exploit the computational efficiency of the WBM, the considered problem geometry should be one of moderate complexity. This observation has initiated the development of a hybrid finite element-wave based modelling approach, in which the ability of the FEM to model any problem, regardless of the geometric complexity, and the computational efficiency of the WBM are combined into one single method. Van Hal [128] and Pluymers [138] have shown the potential of such a hybrid modelling procedure for the analysis of real-life engineering problems with a complex geometrical description.

\section{ACKNOWLEDGEMENTS}

The research work of Bert Pluymers and Bas Van Hal is financed by a scholarship of the Institute for the Promotion of Innovation by Science and Technology in Flanders (IWT).

\section{APPENDIX A}

This appendix gives a detailed description of the WBM system matrices and their derivation.

Substitution of the pressure expansions (67) and (77) and the weighting function expansion (82) into the weighted residual formulation (81) yields

$$
\begin{gathered}
\tilde{\mathbf{p}}_{\mathbf{w}}^{(\alpha) T}\left[\mathbf{C}_{\mathbf{a a}}^{(\alpha, 1)} \mathbf{p}_{\mathbf{w}}^{(1)}+\ldots+\mathbf{C}_{\mathbf{a a}}^{(\alpha, \alpha-1)} \mathbf{p}_{\mathbf{w}}^{(\alpha-1)}+\mathbf{A}_{\mathbf{a a}}^{(\alpha)} \mathbf{p}_{\mathbf{w}}^{(\alpha)}\right. \\
+\mathbf{C}_{\mathbf{a a}}^{(\alpha, \alpha+1)} \mathbf{p}_{\mathbf{w}}^{(\alpha+1)}+\ldots+\mathbf{C}_{\mathbf{a a}}^{\left(\alpha, N_{\Omega}+1\right)} \mathbf{p}_{\mathbf{w}}^{\left(N_{\Omega}+1\right)} \\
\left.-\mathbf{f}_{\mathbf{a}}^{(\alpha, 1)}-\ldots-\mathbf{f}_{\mathbf{a}}^{(\alpha, \alpha-1)}-\mathbf{f}_{\mathbf{a}}^{(\alpha)}-\mathbf{f}_{\mathbf{a}}^{(\alpha, \alpha+1)}-\ldots-\mathbf{f}_{\mathbf{a}}^{\left(\alpha, N_{\Omega}+1\right)}\right]=0
\end{gathered}
$$

The $\left(n_{w}^{(\alpha)} \times n_{w}^{(\alpha)}\right)$ matrix $\mathbf{A}_{\mathbf{a a}}^{(\alpha)}$ is

$$
\mathbf{A}_{\mathbf{a a}}^{(\alpha)}=\mathbf{A}_{\mathbf{v}}^{(\alpha)}+\mathbf{A}_{\mathbf{Z}}^{(\alpha)}+\mathbf{A}_{\mathbf{p}}^{(\alpha)}+\mathbf{A}_{\mathbf{I p}}^{(\alpha)}+\mathbf{A}_{\mathbf{I} \mathbf{v}}^{(\alpha)}+\mathbf{A}_{\mathbf{I} \mathbf{z}}^{(\alpha)}
$$

with

$$
\mathbf{A}_{\mathbf{v}}^{(\alpha)}=\int_{\Gamma_{v}^{(\alpha)}} \frac{j}{\rho \omega} \boldsymbol{\Phi}^{(\alpha) T} \mathbf{n}^{(\alpha) T} \mathbf{B}^{(\alpha)} \mathrm{d} \Omega
$$




$$
\begin{gathered}
\mathbf{A}_{\mathbf{Z}}^{(\alpha)}=\int_{\Gamma_{Z}^{(\alpha)}}\left(\frac{j}{\rho \omega} \boldsymbol{\Phi}^{(\alpha) T} \mathbf{n}^{(\alpha) T} \mathbf{B}^{(\alpha)}-\frac{1}{\bar{Z}_{n}} \boldsymbol{\Phi}^{(\alpha) T} \boldsymbol{\Phi}^{(\alpha)}\right) \mathrm{d} \Gamma \\
\mathbf{A}_{\mathbf{p}}^{(\alpha)}=-\int_{\Gamma_{p}^{(\alpha)}} \frac{j}{\rho \omega} \mathbf{B}^{(\alpha) T} \mathbf{n}^{(\alpha)} \boldsymbol{\Phi}^{(\alpha)} \mathrm{d} \Gamma \\
\mathbf{A}_{\mathbf{I} \mathbf{p}}^{(\alpha)}=-\int_{\Gamma_{I p}^{(\alpha)}} \frac{j}{\rho \omega} \mathbf{B}^{(\alpha) T} \mathbf{n}^{(\alpha)} \boldsymbol{\Phi}^{(\alpha)} \mathrm{d} \Gamma \\
\mathbf{A}_{\mathbf{I v}}^{(\alpha)}=\int_{\Gamma_{I v}^{(\alpha)}} \frac{j}{\rho \omega} \boldsymbol{\Phi}^{(\alpha) T} \mathbf{n}^{(\alpha) T} \mathbf{B}^{(\alpha)} \mathrm{d} \Gamma \\
\mathbf{A}_{\mathbf{I z}}^{(\alpha)}=\int_{\Gamma_{I}^{(\alpha)}}\left(\frac{j}{\rho \omega} \boldsymbol{\Phi}^{(\alpha) T} \mathbf{n}^{(\alpha) T} \mathbf{B}^{(\alpha)}-\frac{1}{\bar{Z}_{n}} \boldsymbol{\Phi}^{(\alpha) T} \boldsymbol{\Phi}^{(\alpha)}\right) \mathrm{d} \Gamma
\end{gathered}
$$

with $\mathbf{B}^{(\alpha)}$ defined as a $\left(2 \times n_{w}^{(\alpha)}\right)$ matrix collecting the gradient components of the acoustic wave functions $\boldsymbol{\Phi}^{(\alpha)}$

$$
\mathbf{B}^{(\alpha)}=\nabla \boldsymbol{\Phi}^{(\alpha)}
$$

The $\left(n_{w}^{(\alpha)} \times n_{a}^{(\beta)}\right)$ matrix $\mathbf{C}_{\mathbf{a a}}^{(\alpha, \beta)}$ is defined as

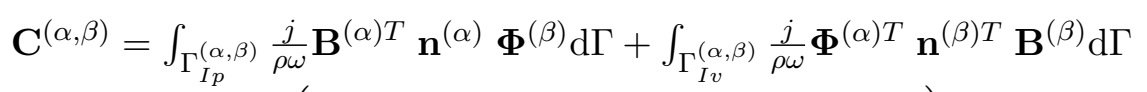

$$
\begin{aligned}
& +\int_{\Gamma_{I z}^{(\alpha, \beta)}}\left(\frac{j}{\rho \omega} \boldsymbol{\Phi}^{(\alpha) T} \mathbf{n}^{(\alpha) T} \mathbf{B}^{(\beta)}+\frac{1}{\bar{Z}_{i n t}} \boldsymbol{\Phi}^{(\alpha) T} \boldsymbol{\Phi}^{(\beta)}\right) \mathrm{d} \Gamma
\end{aligned}
$$

and is a non-zero matrix only if $\Gamma_{I}^{(\alpha, \beta)} \neq \emptyset$. In other words, there is only a non-zero coupling matrix $\mathbf{C}^{(\alpha, \beta)}$ if acoustic subdomain $\Omega^{(\alpha)}$ is adjacent with acoustic subdomain $\Omega^{(\beta)}$.

The $\left(n_{w}^{(\alpha)} \times 1\right)$ vector $\mathbf{f}_{\mathbf{a}}^{(\alpha)}$ is

$$
\mathbf{f}_{\mathbf{a}}^{(\alpha)}=\mathbf{f}_{\mathbf{v}}^{(\alpha)}+\mathbf{f}_{\mathbf{Z}}^{(\alpha)}+\mathbf{f}_{\mathbf{p}}^{(\alpha)}+\mathbf{f}_{\mathbf{I} \mathbf{p}}^{(\alpha)}+\mathbf{f}_{\mathbf{I} \mathbf{v}}^{(\alpha)}+\mathbf{f}_{\mathbf{I} \mathbf{z}}^{(\alpha)}
$$

with

$$
\begin{gathered}
\mathbf{f}_{\mathbf{v}}^{(\alpha)}=\int_{\Gamma_{v}^{(\alpha)}} \boldsymbol{\Phi}^{(\alpha) T}\left(\bar{v}_{n}-\frac{j}{\rho \omega} \mathbf{n}^{(\alpha) T} \nabla \hat{p}_{q}^{(\alpha)}\right) \mathrm{d} \Gamma \\
\mathbf{f}_{\mathbf{Z}}^{(\alpha)}=\int_{\Gamma_{Z}^{(\alpha)}} \boldsymbol{\Phi}^{(\alpha) T}\left(\frac{\hat{p}_{q}^{(\alpha)}}{\bar{Z}_{n}}-\frac{j}{\rho \omega} \mathbf{n}^{(\alpha) T} \boldsymbol{\nabla} \hat{p}_{q}^{(\alpha)}\right) \mathrm{d} \Gamma \\
\mathbf{f}_{\mathbf{p}}^{(\alpha)}=\int_{\Gamma_{p}^{(\alpha)}} \frac{j}{\rho \omega} \mathbf{B}^{(\alpha) T} \mathbf{n}\left(\hat{p}_{q}^{(\alpha)}-\bar{p}\right) \mathrm{d} \Gamma \\
\mathbf{f}_{\mathbf{I} \mathbf{p}}^{(\alpha)}=\int_{\Gamma_{I p}^{(\alpha)}} \frac{j}{\rho \omega} \mathbf{B}^{(\alpha) T} \mathbf{n} \hat{p}_{q}^{(\alpha)} \mathrm{d} \Gamma \\
\mathbf{f}_{\mathbf{I v}}^{(\alpha)}=\int_{\Gamma_{I v}^{(\alpha)}} \boldsymbol{\Phi}^{(\alpha) T}\left(\frac{j}{\rho \omega} \mathbf{n}^{(\alpha) T} \boldsymbol{\nabla} \hat{p}_{q}^{(\alpha)}\right) \mathrm{d} \Gamma \\
\mathbf{f}_{\mathbf{I z}}^{(\alpha)}=\int_{\Gamma_{I}^{(\alpha)}} \boldsymbol{\Phi}^{(\alpha) T}\left(\frac{\hat{p}_{q}^{(\alpha)}}{\bar{Z}_{n}}-\frac{j}{\rho \omega} \mathbf{n}^{(\alpha) T} \boldsymbol{\nabla} \hat{p}_{q}^{(\alpha)}\right) \mathrm{d} \Gamma
\end{gathered}
$$


The $\left(n_{a}^{(\alpha)} \times 1\right)$ vector $\mathbf{f}_{\mathbf{a}}^{(\alpha, \beta)}$ is

$$
\begin{gathered}
\mathbf{f}_{\mathbf{a}}^{(\alpha, \beta)}=-\int_{\Gamma_{I p}^{(\alpha, \beta)}} \frac{j}{\rho \omega} \mathbf{B}^{(\alpha) T} \mathbf{n} \hat{p}_{q}^{(\beta)} \mathrm{d} \Gamma-\int_{\Gamma_{I v}^{(\alpha, \beta)}} \boldsymbol{\Phi}^{(\alpha) T}\left(\frac{j}{\rho \omega} \mathbf{n}^{(\beta) T} \boldsymbol{\nabla} \hat{p}_{q}^{(\beta)}\right) \mathrm{d} \Gamma \\
+\int_{\Gamma_{I z}^{(\alpha, \beta)}} \boldsymbol{\Phi}^{(\alpha) T}\left(-\frac{\hat{p}_{q}^{(\beta)}}{Z_{i n t}}-\frac{j}{\rho \omega} \mathbf{n}^{(\beta) T} \boldsymbol{\nabla} \hat{p}_{q}^{(\beta)}\right) \mathrm{d} \Gamma
\end{gathered}
$$

and is a non-zero vector only if $\Gamma_{I}^{(\alpha, \beta)} \neq \emptyset$.

Since the weighted residual formulation (81) should hold for any weighting function $\tilde{p}^{(\alpha)}$, the expressions between the square brackets in equation (89) must be zero. This yields a set of $n_{w}^{(\alpha)}$ linear equations in the $n_{W}$ unknown wave function contribution factors

$$
\begin{gathered}
{\left[\mathbf{C}_{\mathbf{a a}}^{(\alpha, 1)} \cdots \mathbf{C}_{\mathbf{a a}}^{(\alpha, \alpha-1)} \mathbf{A}_{\mathbf{a}}^{(\alpha)} \mathbf{C}_{\mathbf{a a}}^{(\alpha, \alpha+1)} \cdots \mathbf{C}_{\mathbf{a a}}^{\left(\alpha, N_{\Omega}+1\right)}\right]\left[\begin{array}{c}
\mathbf{p}_{\mathbf{w}}^{(1)} \\
\vdots \\
\mathbf{p}_{\mathbf{w}}^{(\alpha-1)} \\
\mathbf{p}_{\mathbf{w}}^{(\alpha)} \\
\mathbf{p}_{\mathbf{w}}^{(\alpha+1)} \\
\vdots \\
\mathbf{p}_{\mathbf{w}}^{\left(N_{\Omega}+1\right)}
\end{array}\right] .} \\
\\
=\left[\begin{array}{c}
\mathbf{f}_{\mathbf{a}}^{(\alpha, 1)} \\
\vdots \\
\mathbf{f}_{\mathbf{a}}^{(\alpha, \alpha-1)} \\
\mathbf{f}_{\mathbf{a}}^{(\alpha)} \\
\mathbf{f}_{\mathbf{a}}^{(\alpha, \alpha+1)} \\
\vdots \\
\mathbf{f}_{\mathbf{a}}^{\left(\alpha, N_{\Omega}+1\right)}
\end{array}\right]
\end{gathered}
$$

One such matrix equation is obtained for each subdomain. Combination of the $N_{\Omega}+1$ systems yields the acoustic WB model, consisting of $n_{W}$ algebraic equations in the $n_{W}$ unknown wave function contribution factors

$$
\left[\begin{array}{cccc}
\mathbf{A}_{\mathbf{a a}}^{(1)} & \mathbf{C}_{\mathbf{a a}}^{(1,2)} & \cdots & \mathbf{C}_{\mathbf{a a}}^{\left(1, N_{\Omega}+1\right)} \\
\mathbf{C}_{\mathbf{a a}}^{(2,1)} & \mathbf{A}_{\mathbf{a a}}^{(2)} & \cdots & \mathbf{C}_{\mathbf{a a}}^{\left(2, N_{\Omega}+1\right)} \\
& \vdots & & \\
& & & \\
\mathbf{C}_{\mathbf{a a}}^{\left(N_{\Omega}, 1\right)} & \mathbf{C}_{\mathbf{a a}}^{\left(N_{\Omega}, 2\right)} & \cdots & \mathbf{A}_{\mathbf{a a}}^{\left(N_{\Omega}\right)} \\
\mathbf{C}_{\mathbf{a a}}^{\left(N_{\Omega}+1,1\right)} & \mathbf{C}_{\mathbf{a a}}^{\left(N_{\Omega}+1,2\right)} & \cdots & \mathbf{A}_{\mathbf{a a}}^{\left(N_{\Omega}+1\right)}
\end{array}\right]\left[\begin{array}{c}
\mathbf{p}_{\mathbf{w}}^{(1)} \\
\mathbf{p}_{\mathbf{w}}^{(2)} \\
\mathbf{p}_{\mathbf{w}}^{(3)} \\
\vdots \\
\mathbf{p}_{\mathbf{w}}^{\left(N_{\Omega}+1\right)}
\end{array}\right]=\left[\begin{array}{c}
\mathbf{b}^{(1)} \\
\mathbf{b}^{(2)} \\
\mathbf{b}^{(3)} \\
\vdots \\
\mathbf{b}^{\left(N_{\Omega}+1\right)}
\end{array}\right]
$$

with

$$
\mathbf{b}^{(\alpha)}=\mathbf{f}_{\mathbf{a}}^{(\alpha)}+\sum_{\beta=1, \beta \neq \alpha}^{N_{\Omega}+1} \mathbf{f}_{\mathbf{a}}^{(\alpha, \beta)} .
$$

The WB matrix equation is denoted in a condensed form as

$$
\mathbf{A}_{\text {aa }} \mathbf{p}_{\mathbf{w}}=\mathbf{b} .
$$




\section{References}

1 P. Morse and K. Ingard. Theoretical acoustics. McGraw-Hill, New York, 1968.

2 A. Pierce. Acoustics: An Introduction to Its Physical Principles and Applications. McGraw-Hill series in mechanical engineering, McGraw-Hill, 1981.

3 O.C. Zienkiewicz, R.L. Taylor, J.Z. Zhu, and P. Nithiarasu. The Finite Element Method - The three volume set. Butterworth-Heinemann, 6th edition, 2005.

4 F. Ihlenburg. Finite Element Analysis of Acoustic Scattering. Volume 132 of Applied Mathematical Sciences, Springer, 1998.

5 C.A. Brebbia, J.C.F. Telles, and L.C.L. Wrobel. Boundary Element Techniques: Theory and Applications in Engineering. Springer-Verlag, 1984.

6 S. Kirkup. The Boundary Element Method in Acoustics. Integrated Sound Software, 1998.

7 O. Von Estorff. Boundary Elements in Acoustics: Advances and Applications. WITpress, 2000.

8 O.C. Zienkiewicz. Achievements and some unsolved problems of the finite element method. Int. J. Num. Methods Engrg., 47:9-28, 2000.

9 Proceedings of the Fifth World Congress on Computational Mechanics, Vienna, Austria, 2002.

10 Proceedings of the European Congress on Computational Methods in Applied Sciences and Engineering, Jyvaskyla, Finland, 2004.

11 Proceedings of the 2004 International Conference on Noise and Vibration Engineering, Leuven, Belgium, 2004.

12 Proceedings of the 13th International Congress on Sound and Vibration, Vienna, Austria, 2006.

13 Proceedings of the 2006 International Conference on Noise and Vibration Engineering, Leuven, Belgium, 2006.

14 W. Desmet. Mid-frequency vibro-acoustic modelling: challenges and potential solutions. In Proceedings of the 2002 International Conference on Noise and Vibration Engineering, pages 835-862, Leuven, Belgium, 2002.

15 I. Harari. A survey of finite element methods for time-harmonic acoustics. Comput. Methods Appl. Mech. Engrg., 195:1594-1607, 2006.

16 L.L. Thompson. A review of finite element methods for time-harmonic acoustics. $J$. Acoustical Soc. Am., 119:1315-1330, 2006.

17 E. Trefftz. Ein Gegenstück zum Ritzschen Verfahren. In Proceedings of the $2^{\text {nd }}$ International Congress on Applied Mechanics, pages 131-137, Zürich, Switzerland, 1926.

18 J. Jirousek and A. Wróblewski. T-elements: State of the Art and Future Trends. Archives Comput. Methods Engrg., 3:323-434, 1996. 
19 E. Kita and N. Kamiya. Trefftz method: an overview. Adv. Engrg. Software, 24:3-12, 1995.

20 W. Desmet, B. Van Hal, P. Sas, and D. Vandepitte. A computationally efficient prediction technique for the steady-state dynamic analysis of coupled vibro-acoustic systems. Adv. Engrg. Software, 33:527-540, 2002.

21 B. Van Hal, W. Desmet, D. Vandepitte, and P. Sas. A coupled finite element - wave based approach for the steady-state dynamic analysis of acoustic systems. J. Comput. Acoust., 11:255-283, 2003.

22 B. Pluymers, W. Desmet, D. Vandepitte, and P. Sas. Application of an efficient wave based prediction technique for the analysis of vibro-acoustic radiation problems. $J$. Comput. Appl. Math., 168:353-364, 2004.

23 B. Pluymers, W. Desmet, D. Vandepitte, and P. Sas. On the Use of a Wave Based Prediction Technique for Steady-State Structural-Acoustic Radiation Analysis. J. Comput. Modeling in Engineering \& Sciences, 7(2):173-184, 2005.

24 B. Van Hal, W. Desmet, and D. Vandepitte. Hybrid finite element - wave based method for steady-state interior structural-acoustic problems. Computers \& Structures, 83: 167-180, 2005.

25 D. Colton and R. Kress. Inverse acoustic and electromagnetic scattering theory. Springer-Verlag, 2nd edition, 1998.

26 W. Desmet. Boundary element method in acoustics. Course notes ISAAC17: Seminar on Advanced Techniques in Applied and Numerical Acoustics, Leuven, Belgium, September 2006.

27 J.P. Wolf and C. Song. Finite element modelling of unbounded media. John Wiley \& Sons, Chichester, 1996.

28 L.L. Thompson and P.M. Pinsky. Acoustics. Encyclopedia of Computational Mechanics, 2004.

29 J.J. Shirron and I. Babuška. A comparison of approximate boundary conditions and infinite element methods for exterior Helmholtz problems. Comput. Methods Appl. Mech. Engrg., 164:121-139, 1998.

30 D. Givoli. High-order local non-reflecting boundary conditions: a review. Wave Motion, 39:319-326, 2004.

31 J.B. Keller and D. Givoli. Exact non-reflecting boundary conditions. J. Comput. Physics, 82:172-192, 1989.

32 I. Harari, I. Patlashenko, and D. Givoli. Dirichlet-to-Neumann maps for unbounded wave guides. J. Comput. Physics, 143:200-223, 1998.

33 D.P. Nicholls and N. Nigam. Exact non-reflecting boundary conditions on general domains. J. Comput. Physics, 194:278-303, 2004.

34 P. Bettess. Infinite Elements. Penshaw Press, 1992.

$35 \mathrm{~K}$. Gerdes. A review of infinite element methods for exterior Helmholtz problems. $J$. Comput. Acoust., 8:43-62, 2000. 
36 D.S. Burnett and R.L. Holford. Prolate and oblate spheroidal acoustic infinite elements. Comput. Methods Appl. Mech. Engrg., 158:117-141, 1998.

37 D.S. Burnett and R.L. Holford. An ellipsoidal acoustic infinite element. Comput. Methods Appl. Mech. Engrg., 164:49-76, 1998.

38 R.J. Astley, G.J. Macaulay, and J.P. Coyette. Mapped wave envelope elements for acoustical radiation and scattering. J. Sound \& Vibration, 170:97-118, 1994.

39 R.J. Astley, G.J. Macaulay, J.P. Coyette, and L. Cremers. Three-dimensional waveenvelope elements of variable order for acoustic radiation and scattering. Part I. Formulation in the frequency domain. J. Acoustical Soc. Am., 103:49-63, 1998.

40 R.J. Astley and J.P. Coyette. The performance of spheroidal infinite elements. Int. J. Num. Methods Engrg., 52:1379-1396, 2001.

41 J.-P. Berenger. A Perfectly Matched Layer for the Absorption of Electromagnetic Waves. J. Comput. Physics, 114:185-200, 1994.

42 F. Collino and P. Monk. The perfectly matched layer in curvilinear coordinates. SIAM J. Sci. Comput., 19:2061-2090, 1998.

$43 \mathrm{Ph}$. Bouillard and F. Ihlenburg. Error estimation and adaptivity for the finite element method in acoustics: 2D and 3D applications. Comput. Methods Appl. Mech. Engrg., 176:147-163, 1999.

44 G. Bartsch and C. Wulf. Adaptive multigrid for Helmholtz problems. J. Comput. Acoustics, 11:341-350, 2003.

45 I. Harari and D. Avraham. High-order finite element methods for acoustic problems. J. Comput. Acoustics, 5:33-51, 1997.

46 S. Dey, D.K. Datta, J.J. Shirron, and M.S. Shephard. p-Version FEM for structural acoustics with a posteriori error estimation. Comput. Methods Appl. Mech. Engrg., 195:1946-1957, 2006.

47 D.S. Burnett. 3D Structural acoustic modelling with hp-adaptive finite elements. In Proceedings of the XXI International Congress of Theoretical and Applied Mechanics ICTAM, Warsaw, Poland, 2004.

48 M. Ainsworth and T.J. Oden. Posteriori error estimation in finite element analysis. Comput. Methods Appl. Mech. Engrg., 142:1-88, 1997.

49 O.C. Zienkiewicz. The background of error estimation and adaptivity in finite element computations. Comput. Methods Appl. Mech. Engrg., 195:207-213, 2006.

50 J.T. Oden, S. Prudhomme, and L. Demkowicz. A posteriori error estimation for acoustic wave propagation. Archives Comput. Methods Engrg., 12:343-390, 2005.

51 L. L. Thompson and P. Kunthong. A residual based variational method for reducing dispersion error in finite element methods. In Proceedings of the 2005 ASME International Mechanical Engineering Congress and Exposition, pages Paper IMECE200580551, Orlando, Florida, USA, 2005.

52 M.N. Guddati and B. Yue. Modified integration rules for reducing dispersion error in finite element methods. Comput. Methods Appl. Mech. Engrg., 193:275-287, 2004. 
53 I. Duff. Direct Methods. Technical Report RAL-TR-1998-054, Rutherford Appleton Laboratory, Oxon, USA, 1998.

54 Y. Saad. Iterative methods for sparse linear systems. SIAM, second edition, 2003.

55 R.R. Craig Jr. Structural Dynamics: An Introduction to Computer Methods. John Wiley \& Sons, 1981.

56 J.K. Bennighof and M.F. Kaplan. Frequency Window Implementation of Adaptive Multi-Level Substructuring. J. Vibrations \& Acoustics, 120:409-418, 1998.

57 J.K. Bennighof, M.F. Kaplan, M.B. Muller, and M. Kim. Meeting the NVH Computational Challenge: Automated Multi-Level Substructuring. In Proceedings of the International Modal Analysis Conference XVIII, pages 909-915, San Antonio, USA, 2000 .

58 A. Kropp and D. Heiserer. Efficient broadband vibro-acoustic analysis of passenger car bodies using an FE-based component mode synthesis approach. J. Comput. Acoustics, 11:139-157, 2003.

59 R. Stryczek, A. Kropp, and S. Wegner. Vibro-acoustic computations in the midfrequency range: efficiency, evaluation and validation. In Proceedings of the International Conference on Noise and Vibration Engineering ISMA2004, pages 1603-1612, Leuven, Belgium, 2004.

60 B. Després. Méthodes de décomposition de domaine pour les problémes de propagation d'ondes en régime harmonique. PhD thesis, Paris IX Dauphine, Paris, France, 1991.

61 X. Cai, M. Casarin, F. Elliott Jr., and O. Widlund. Overlapping Schwartz Algorithms for Solving Helmholtz's Equation. Contemporary Mathematics, 218:391-399, 1998.

62 C. Farhat, A. Macedo, and M. Lesoinne. A two-level domain decomposition method for the iterative solution of high frequency exterior Helmholtz problems. Numerische Mathematik, 85:283-308, 2000.

63 C. Farhat and F.-X. Roux. A method of finite element tearing and interconnecting nad its parallel solution algorithm. Int. J. Num. Methods Engrg., 32:1205-1227, 1991.

64 C. Farhat, A. Macedo, M. Lesoinne, F.-X. Roux, F. Magoules, and A. de La Bourdonnaie. Two-level domain decomposition methods with Lagrange multipliers for the fast iterative solution of acoustic scattering problems. Comput. Methods Appl. Mech. Engrg., 184:213-239, 2000.

65 D.K. Datta, S. Dey, and J.J. Shirron. Scalable Three-Dimensional Acoustics Using hpfinite/infinite elements and FETI-DP. In Proceedings of the 16th International Domain Decomposition Conference, New York, USA, 2005.

66 F. Magoules. Decomposition of Domains in Acoustics, http://www.iecn.u-nancy. $\mathrm{fr} /$ 〜magoules/pai/greenwich/aeroacoustics2/, 2006.

67 J. Mandel. An iterative substructuring method for coupled fluid-solid acoustic problems. J. Comput. Physics, 177:95-116, 2002.

68 I. Harari and F. Magoules. Numerical investigations of stabilized finite element computations for acoustics. Wave Motion, 39:339-349, 2004. 
69 I. Harari and T.J.R. Hughes. Galerkin/least-squares finite element methods for the reduced wave equation with non-reflecting boundary conditions in unbounded domains. Comput. Methods Appl. Mech. Engrg., 98:411-454, 1992.

70 L.L. Thompson and P.M. Pinsky. A Galerkin Least Squares Finite Element Method for the Two-Dimensional Helmholtz Equation. Int. J. Num. Methods Engrg., 38:371-397, 1995.

71 L.P. Franca and E.G. Dutra do Carmo. Galerkin gradient least-squares method. Comput. Methods Appl. Mech. Engrg., 74:41-54, 1989.

72 I. Harari. Reducing spurious dispersion, anisotropy and reflection in finite element analysis of time-harmonic acoustics. Comput. Methods Appl. Mech. Engrg., 140:39-58, 1997.

73 I. Babuška, F. Ihlenburg, E.T. Paik, and S.A. Sauter. A generalized finite element method for solving the Helmholtz equation in two dimensions with minimal pollution. Comput. Methods Appl. Mech. Engrg., 128:325-359, 1995.

74 I. Babuška and S.A. Sauter. Is the Pollution Effect of the FEM Avoidable for the Helmholtz Equation Considering High Wave Numbers? SIAM J. Num. An., 34:23922423, 1997.

75 B. Yue and M.N. Guddati. Highly accurate local mesh-dependent augmented galerkin (L-MAG) FEM for simulation of time-harmonic wave propagation. In Proceedings of the 16th ASCE Engineering Mechanics Conference, Seattle, USA, 2003.

76 J.M. Melenk. On generalized finite element methods. PhD thesis, University of Maryland at College Park, 1995.

77 I. Babuška and J.M. Melenk. The partition of unity method. Int. J. Num. Methods Engrg., 40:727-758, 1997.

78 J.M. Melenk and I. Babuška. The partition of unity finite element method: basic theory and applications. Comput. Methods Appl. Mech. Engrg., 139:289-314, 1996.

79 O. Laghrouche and P. Bettess. Short wave modelling using special finite elements. J. Comput. Acoustics, 8:189-210, 2000.

80 T. Strouboulis, I. Babuška, and K. Copps. The design and analysis of the Generalized Finite Element Method. Comput. Methods Appl. Mech. Engrg., 181:43-69, 2000.

81 T. Strouboulis, K. Copps, and I. Babuška. The generalized finite element method. Comput. Methods Appl. Mech. Engrg., 190:4081-4193, 2001.

82 T. Belytschko, Y. Krongauz, D. Organ, M. Fleming, and P. Krysl. Meshless methods: An overview and recent developments. Comput. Methods Appl. Mech. Engrg., 139: 3-47, 1996.

$83 \mathrm{Ph}$. Bouillard and S. Suleau. Element-Free Galerkin solutions for Helmholtz problems: formulation and numerical assessment of the polution effect. Comput. Methods Appl. Mech. Engrg., 162:317-335, 1998.

84 S. Suleau, A. Deraemaeker, and Ph. Bouillard. Dispersion and pollution of meshless solutions for the Helmholtz equation. Comput. Methods Appl. Mech. Engrg., 190:639$657,2000$. 
85 V. Lacroix, Ph. Bouillard, and P. Villon. An iterative defect-correction type meshless method for acoustics. Int. J. Num. Methods Engrg., 57:2131-2146, 2003.

86 P. Lancaster and K. Salkauskas. Surfaces generated by moving least squares methods. Mathematics of Computation, 37:141-158, 1981.

87 T.J.R. Hughes. Multiscale phenomena: Green's functions, Dirichlet-to-Neumann formulation, subgrid scale models, bubbles and the origins of stabilized methods. Comput. Methods Appl. Mech. Engrg., 127:387-401, 1995.

88 T.J.R. Hughes, G.R. Feijoo, L. Mazzei, and J.-B. Quincy. The variational multiscale method - a paradigm for computational mechanics. Comput. Methods Appl. Mech. Engrg., 166:3-24, 1998.

89 L. Franca, C. Farhat, A. Macedo, and M. Lesoinne. Residual-free bubbles for Helmholtz equation. Int. J. Num. Methods Engrg., 40:4003-4009, 1997.

90 F. Brezzie, L.P. Franca, T.J.R. Hughes, and A. Russo. $b=\int g$. Comput. Methods Appl. Mech. Engrg., 145:329-339, 1997.

91 J.L. Cipolla. Subgrid modeling in a Galerkin method for the Helmholtz equation. Comput. Methods Appl. Mech. Engrg., 177:35-49, 1999.

92 A.A. Oberai and P.M. Pinsky. A multiscale finite element method for the Helmholtz equation. Comput. Methods Appl. Mech. Engrg., 154:281-297, 1998.

93 C. Farhat, I. Harari, and L.P. Franca. The discontinuous enrichment method. Comput. Methods Appl. Mech. Engrg., 190:6455-6479, 2001.

94 C. Farhat, I. Harari, and U. Hetmaniuk. The discontinuous enrichment method for multiscale analysis. Comput. Methods Appl. Mech. Engrg., 192:3195-3209, 2003.

95 S. Marburg. Six boundary elements per wavelength: is that enough? J. Comput. Acoust., 10:25-51, 2002.

96 I. Harari and T.J.R. Hughes. A cost comparison of boundary element and finite element methods for problems of time-harmonic acoustics. Comput. Methods Appl. Mech. Engrg., 98:77-102, 1992.

97 D.W. Herrin, F. Martinus, T.W. Wu, and A.F. Seybert. An assessment of the high frequency boundary element and Rayleigh integral approximations. Appl. Acoust., 67: 819-833, 2006.

98 A.B. Nagy, P. Fiala, F. Marki, F. Augustinovicz, G. Degrande, S. Jacobs, and D. Brassenx. Prediction of interior noise in buildings generated by underground rail traffic. J. Sound \& Vibration, 293:680-690, 2006.

99 F.X. DeBiesme, J.W. Verheij, and G. Verbeek. Lumped parameter BEM for faster calculations of sound radiation from vibrating structures. In Proceedings of the Tenth International Congress on Sound and Vibration, pages 1515-1522, Stockholm, Sweden, 2003.

100 F.X. DeBiesme and J.W. Verheij. Speed gains of the lumped parameter boundary element method for vibroacoustics. In Proceedings of the International Conference on Noise and Vibration Engineering ISMA2004, pages 3765-3778, Leuven, Belgium, 2004. 
101 J.B. Fahnline and G.H. Koopman. A lumped parameter model for the acoustic power output from a vibrating structure. J. Acoustical Soc. Am., 100:3539-3547, 1996.

102 E. Perrey-Debain, J. Trevelyan, and P. Bettess. Wave boundary elements: a theoretical overview presenting applications in scattering of short waves. J. Eng. Ana. Bound., 28:131-141, 2004.

103 E. Perrey-Debain, O. Laghrouche, P. Bettess, and J. Trevelyan. Plane wave basis finite elements and boundary elements for three dimensional wave scattering. Phil. Trans. R. Soc. A, 362:561-577, 2004.

104 N.S. Chandler-Wilde, S. Langdon, and L. Ritter. A high-wavenumber boundaryelement method for an acoustic scattering problem. Phil. Trans. R. Soc. A, 362: 647-671, 2004.

105 S. Arden, N.S. Chandler-Wilde, and S. Langdon. A collocation method for high frequency scattering by convex polygons. Technical Report Numerical Analysis Report 7/05, Reading University, 2005.

106 D. Huybrechs and S. Vandewalle. On the evaluation of highly oscillatory integrals by analytic continuation. SIAM J. Numer. Anal., 44:1026-1048, 2006.

107 D. Huybrechs and S. Vandewalle. The construction of cubature rules for multivariate highly oscillatory integrals. Technical Report Technisch rapport TW-442, K.U.Leuven, 2005 .

108 D. Huybrechs and S. Vandewalle. A sparse discretisation for integral equation formulations of high frequency scattering problems. Technical Report Technisch rapport TW-447, K.U.Leuven, 2006.

109 D. Huybrechs. Multiscale and hybrid methods for the solution of oscillatory integral equations. PhD thesis, KULeuven, Leuven, Belgium, 2006. http://www.cs.kuleuven. be $/$ daan/phd/thesis.pdf.

110 T. Sakuma and Y. Yasuda. Fast multipole boundary element method for large-scale steady-state sound field analysis. Part I: setup and validation. Acustica, 88:513-525, 2002 .

111 Y. Yasuda and T. Sakuma. Fast multipole boundary element method for large-scale steady-state sound field analysis. Part I: examination of numerical items. Acustica, 89: $28-38,2002$.

112 S. Schneider. Application of fast methods for acoustic scattering and radiation problems. J. Computational Acoust., 11:387-401, 2003.

113 M. Fischer, U. Gauger, and L. Gaul. A multipole Galerkin boundary element method for acoustics. Eng. Ana. Bound., 28:155-162, 2004.

114 Y. Yasuda and T. Sakuma. An effective setting of hierarchical cell structure for the fast multipole boundary element method. J. Computational Acoust., 13:47-70, 2005.

115 Y. Yasuda and T. Sakuma. A technique for plane-symmetric sound field analysis in fast multipole boundary element method. J. Computational Acoust., 13:71-86, 2005.

116 M. Fischer and L. Gaul. Fast BEM-FEM mortar coupling for acoustic-structure interaction. Int. J. Num. Methods Engrg., 62:1677-1690, 2005. 
117 Y.K. Cheung, W.G. Jin, and O.C. Zienkiewicz. Solution of Helmholtz problems by Trefftz method. Int. J. Num. Methods Engrg., 32:63-78, 1991.

118 I. Herrera. Boundary Methods: an Algebraic Theory. Pitman Adv. Publ. Program, London, UK, 1984.

119 P. Masson, E. Redon, J.-P. Priou, and Y. Gervais. The application of the Trefftz method for acoustics. In Proceedings of the Third International Congress on Sound and Vibration, pages 1809-1816, Montreal, Canada, 1994.

120 J. Sladek, V. Sladek, and R. Van Keer. Global and local Trefftz boundary integral formulations for sound vibration. Adv. Engrg. Software, 33:469-476, 2002.

121 A.P. Zielinski and O.C. Zienkiewicz. Generalized finite element analysis with Tcomplete boundary solution functions. Int. J. Num. Methods Engrg., 21:509-528, 1985.

122 A.P. Zielinski and I. Herrera. Trefftz method: fitting boundary conditions. Int. J. Num. Methods Engrg., 24:871-891, 1987.

123 Q.-H. Qin. The Trefftz Finite and Boundary Element Method. WIT Press, Southampton, UK, 2000.

124 V. Kompis and M. Stiavnicky. Trefftz functions in FEM, BEM and meshless methods. In Proceedings of the 10th International Conference on Numerical Methods for Continuum Mechanics $\&$ the 4th International Workshop on Trefftz methods, Zilina, Slovak Republic, 2005.

125 M. Ochmann. The source simulation technique for acoustic radiation problems. Acustica, 81:512-527, 1995.

126 O. Cessenat and B. Deprés. Application of an ultra weak variational formulation of elliptic pdes to the two-dimensional Helmholtz problem. SIAM J. Num. An., 35:255299, 1998.

127 E.J. Kansa. Multiquadrics: A scattered data approximation scheme with applications to computational fluid-dynamics-II solutions to parabolic, hyperbolic and elliptic partial differential equations. Computers $\& 5$ Mathematics with Applications, 19:147-161, 1990.

128 B. Van Hal. Automation and performance optimization of the wave based method for interior structural-acoustic problems. PhD thesis, KULeuven, Leuven, Belgium, 2004. http://www.mech.kuleuven.be/dept/resources/docs/vanhal.pdf.

129 C. Farhat, I. Harari, and U. Hetmaniuk. A discontinuous Galerkin method with Lagrange multipliers for the solution of Helmholtz problems in the mid-frequency range. Comput. Methods Appl. Mech. Engrg., 192:1389-1419, 2003.

130 R. Tezaur and C. Farhat. Three-dimensional discontinuous Galerkin elements with plane waves and Lagrange multipliers for the solution of mid-frequency Helmholtz problems. Int. J. Num. Methods Engrg., 66:796-815, 2006.

131 J. Jirousek and A. Wróblewski. Least-squares T-elements: equivalent FE and BE forms of substructure oriented boundary solution approach. Communication Num. Methods Engrg., 10:21-32, 1994. 
132 M. Stojek. Finite T-elements for the Poisson and Helmholtz equations. PhD thesis, École Polytechnique Fédérale de Lausanne, Lausanne, Switzerland, 1996.

133 M. Stojek. Least-squares Trefftz-type elements for the Helmholtz equation. Int. J. Num. Methods Engrg., 41:831-849, 1998.

134 P. Monk and D.-Q. Wang. A least-squares method for the Helmholtz equation. Comput. Methods Appl. Mech. Engrg., 175:121-136, 1999.

135 H. Riou, P. Ladeveze, and P. Rouch. Extension of the variational theory of complex rays to shells for medium-frequency vibrations. J. Sound $\&$ Vibration, 272:341-360, 2004 .

136 W. Desmet. A wave based prediction technique for coupled vibro-acoustic analysis. $\mathrm{PhD}$ thesis, KULeuven, Leuven, Belgium, 1998. http://people.mech.kuleuven.ac.be/ wdesmet/desmet_phd_thesis.pdf.

137 B. Pluymers, C. Vanmaele, W. Desmet, D. Vandepitte, and P. Sas. Application of a novel wave based prediction technique for acoustic cavity analysis. In Proceedings of the 30th German Convention on Acoustics (DAGA) together with the 7th Congrs Francais d'Acoustique (CFA), pages 313-314, Strasbourg, France, 2004.

138 B. Pluymers. Wave based modelling methods for steady-state vibro-acoustics. $\mathrm{PhD}$ thesis, KULeuven, Leuven, Belgium, 2006. http://people.mech.kuleuven.be/ 〜bpluymer/docs/thesis_bpluymers.pdf.

139 B. Pluymers, C. Vanmaele, W. Desmet, and D. Vandepitte. Application of a hybrid finite element - Trefftz approach for acoustic analysis. In Proceedings of the 10th International Conference on Numerical Methods for Continuum Mechanics 85 the 4 th International Workshop on Trefftz methods, Zilina, Slovak Republic, 2005.

140 B. Van Genechten, B. Pluymers, C. Vanmaele, D. Vandepitte, and W. Desmet. On the coupling of Wave Based models with modally reduced Finite Element models for structural-acoustic analysis. In Proceedings of the International Conference on Noise and Vibration Engineering ISMA2006, pages 2383-2404, Leuven, Belgium, 2006.

141 J.D. Benamou and B. Depres. A domain decomposition method for the Helmholtz equation and related optimal control problems. J. Comput. Physics, 136:68-82, 1997.

142 G.H. Koopmann, L. Song, and J.B. Fahnline. A method for computing acoustic fields based on the principle of wave superposition. J. Acoustical Soc. Am., 86:2433-2438, 1989 .

143 B Stupfel, A. Lavie, and J.H. Decarpigny. Combined integral equation formulation and null-field method for the exterior acoustic problem. J. Acoustical Soc. Am., 83: 927-941, 1988.

144 S.Y. Reutskiy. The method of fundamental solutions for Helmholtz eigenvalue problems in simply and multiply connected domains. Eng. Ana. Bound., 30:150-159, 2006.

145 M. Ochmann. The full-field equations for acoustic radiation and scattering. J. Acoustical Soc. Am., 105:2574-2584, 1999.

146 M. Ochmann. The complex equivalent source method for sound propagation over an impedance plane. J. Acoustical Soc. Am., 116:3304-3311, 2004. 
147 G. Pavic. An engineering technique for the computation of sound radiation by vibrating bodies using substitute sources. Acustica, 91:1-16, 2005.

148 G. Pavic. A technique for the computation of sound radiation by vibrating bodies using multipole substitute sources. In Proceedings of the International Congress on Noise and Vibration Emerging Methods (NOVEM2005), Saint-Raphael, France, 2005.

149 L. Bouchet, T. Loyau, N. Hamzaoui, and C. Boisson. Calculation of acoustic radiation using equivalent-sphere methods. J. Acoustical Soc. Am., 107:2387-2397, 2000.

150 E. Reboul, J. Perret-Liaudet, and A. Le Bot. Vibroacoustic prediction of mechanisms using a hybrid method. In Proceedings of the International Congress on Noise and Vibration Emerging Methods (NOVEM2005), Saint-Raphael, France, 2005.

151 D.W. Herrin, T.W. Wu, and A.F. Seybert. The energy source simulation method. J. Sound \& Vibration, 278:135-153, 2004.

152 W. Chen. Symmetric boundary knot method. Eng. An. Bound., 26:489-494, 2002.

153 M.A. Goldbert and C.S. Chen. The MFS for potential, Helmholtz and diffusion problems, Chapter 4, Boundary Integral Methods: Numerical and Mathematical Aspects, ed. M.A. Golberg. WIT Press and Computational Mechanics Publ. Boston, Southampton, 1999.

154 C.J.S. Alves and S.S. Valtchev. Numerical comparison of two meshfree methods for acoustic wave scattering. Eng. An. Bound., 29:371-382, 2005.

155 N. Limic. Galerkin-Petrov method for Helmholtz equation on exterior problems. Glasnik Mathematicki, 36:245-260, 1981.

156 G. Fairweather and A. Kargeorghis. The MFS for elliptic BVPs. Advanced Computational Mathematics, 9:69-95, 1998.

157 C.S. Chen, M.A. Golberg, and R.S. Schaback. Transformation of Domain Effects to the Boundary, Chapter 8: Recent developments of the dual reciprocity method using compactly supported radial basis functions, ed. Y.F. Rashed. WIT Press, Southampton, 2002 .

158 T. Huttunen, P. Gamallo, and R.J. Astley. Comparison of two wave element methods for the Helmholtz problem. Comput. Methods Appl. Mech. Engrg., 2006.

159 P. Sas, W. Desmet, B. Van Hal, and B. Pluymers. On the use of a wave based prediction technique for vehicle interior acoustics. In Proceedings of the Styrian Noise, Vibration $\mathcal{G}^{3}$ Harshness Congress: Integrated Vehicle Acoustics and Comfort, pages 175-185, Graz, Austria, 2001.

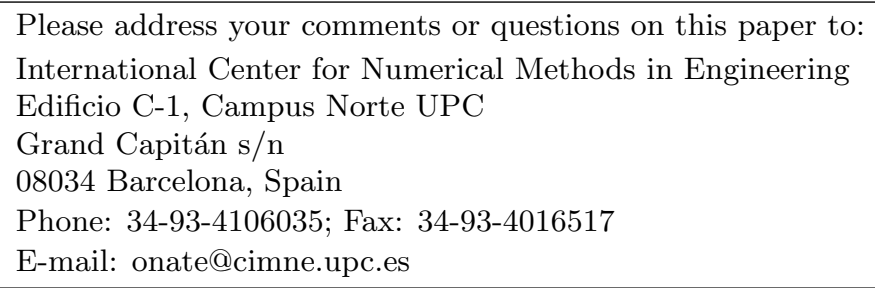

